\title{
Variational inequality approach to enforcing the non-negative constraint for advection-diffusion equations
}

AN E-PRINT OF THE PAPER IS AVAILABle ON ARXIV: 1611.08758.

Authored BY

J. CHANG

Graduate Student, University of Houston.

K. B. NAKShatrala

Department of Civil \&5 Environmental Engineering

University of Houston, Houston, Texas 77204-4003.

phone: +1-713-743-4418, e-mail: knakshatrala@uh.edu

website: http://www.cive.uh.edu/faculty/nakshatrala

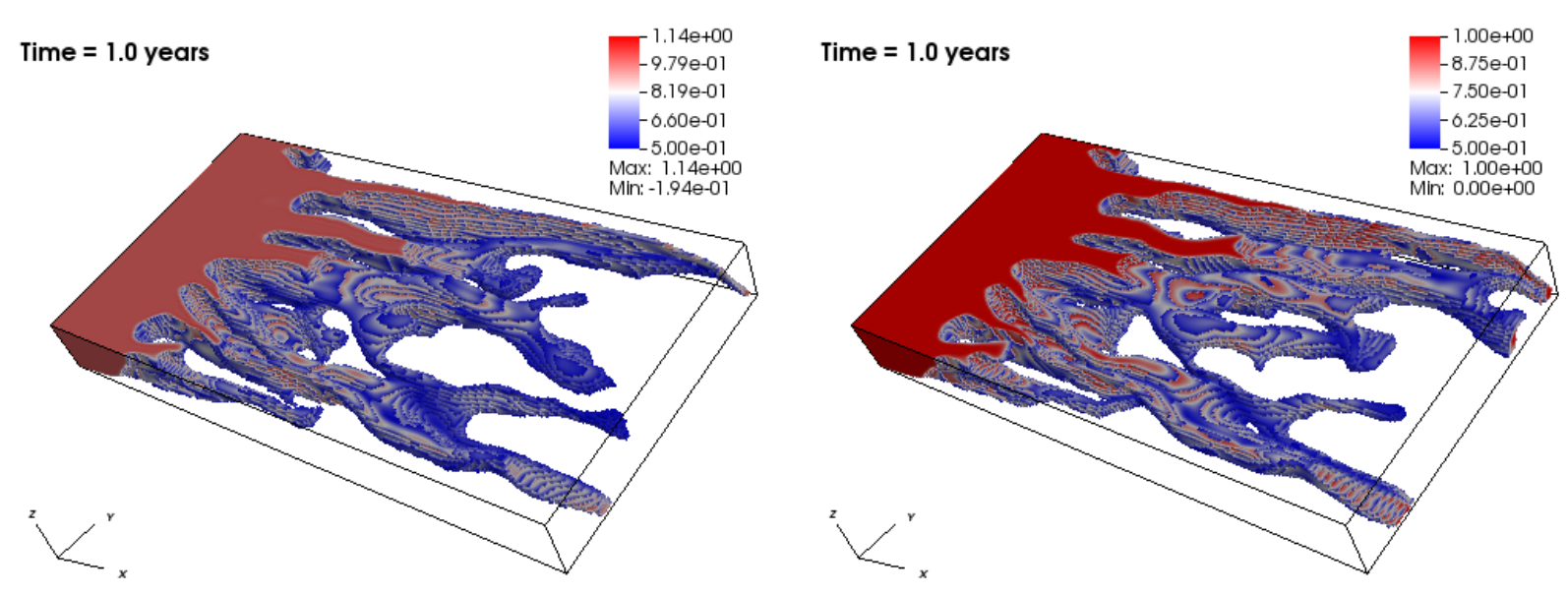

These figures depict the concentration profiles of the advection-diffusion equation under the Discontinuous Galerkin formulation (left) and the Discontinuous Galerkin formulation with bounded constraints enforced through a variational inequality (right). Only the regions where concentrations meet the threshold of 0.5 and above are shown.

2016

Computational \& Applied Mechanics Laboratory

(C) 2016. This manuscript version is made available under the Elsevier user license http://www.elsevier.com/open-access/userlicense/1.0/ 


\title{
Variational inequality approach to enforcing the non-negative constraint for advection-diffusion equations
}

\author{
J. Chang, and K. B. Nakshatrala \\ Department of Civil and Environmental Engineering, University of Houston. \\ Correspondence to: knakshatrala@uh.edu
}

\begin{abstract}
Predictive simulations are crucial for the success of many subsurface applications, and it is highly desirable to obtain accurate non-negative solutions for transport equations in these numerical simulations. To this end, optimization-based methodologies based on quadratic programming (QP) have been shown to be a viable approach to ensuring discrete maximum principles and the non-negative constraint for anisotropic diffusion equations. In this paper, we propose a computational framework based on the variational inequality (VI) which can also be used to enforce important mathematical properties (e.g., maximum principles) and physical constraints (e.g., the non-negative constraint). We demonstrate that this framework is not only applicable to diffusion equations but also to non-symmetric advection-diffusion equations. An attractive feature of the proposed framework is that it works with with any weak formulation for the advection-diffusion equations, including single-field formulations, which are computationally attractive. A particular emphasis is placed on the parallel and algorithmic performance of the VI approach across large-scale and heterogeneous problems. It is also shown that QP and VI are equivalent under certain conditions. State-of-the-art QP and VI solvers available from the PETSc library are used on a variety of steady-state 2D and 3D benchmarks, and a comparative study on the scalability between the $\mathrm{QP}$ and VI solvers is presented. We then extend the proposed framework to transient problems by simulating the miscible displacement of fluids in a heterogeneous porous medium and illustrate the importance of enforcing maximum principles for these types of coupled problems. Our numerical experiments indicate that VIs are indeed a viable approach for enforcing the maximum principles and the non-negative constraint in a large-scale computing environment. Also provided are Firedrake project files as well as a discussion on the computer implementation to help facilitate readers in understanding the proposed framework.
\end{abstract}

\section{A list of abbreviations}

\begin{tabular}{|l|l|}
\hline ABC & Arnold-Beltrami-Childress \\
CG & Conjugate Gradient method \\
DG & Discontinuous Galerkin \\
GAL & (Continuous) Galerkin \\
GMRES & Generalized Minimal Residual method \\
KSP & Krylov subspace iterative solver \\
\hline
\end{tabular}

Key words and phrases. anisotropy; variational inequalities; quadratic programming; non-negative solutions; maximum principles; parallel computing; advection-diffusion equations; miscible displacement. 


\begin{tabular}{|l|l|} 
MCP & Mixed complementarity problem \\
MP & Minimization problem \\
PETSc & Pessage Passing Interface \\
QP & Quadratic programming \\
QP - TRON & Trust region Newton method \\
SP & Strong problem \\
SUPG & Streamlined Upwind Petrov-Galerkin \\
TAO & Toolkit for Advanced Optimization [Munson et al., 2014] \\
VI & Variational Inequality \\
VI - SS & Semi-smooth method \\
VI - RS & Reduced-space active-set method \\
WF & Weak formulation \\
\hline
\end{tabular}

\section{INTRODUCTION AND MOTIVATION}

This paper presents a numerical methodology based on variational inequalities for anisotropic diffusion and advection-diffusion equations that satisfies discrete maximum principles, meets the non-negative constraint, and is well-suited for solving large-scale problems using parallel computing. We now provide a motivation behind the current work, a discussion on prior works, and an outline of our approach highlighting the significance of the work presented herein.

The diffusion and advection-diffusion equations are important partial differential equations which are commonly used to model flow and transport of chemical species in porous media. Some of the applications include subsurface remediation [EPA, 2004; Harp and Vesselinov, 2013; Heikoop et al., 2014] and transport of radionuclides [Hammond and Lichtner, 2010; Genty and Potier, 2011]. Since these important problems are not analytically tractable, one needs to rely on predictive numerical simulations. An important aspect in a predictive simulation of these equations is to satisfy the non-negative constraint of concentration of chemical species.

Research efforts over the years have successfully created numerical models and discretization for these equations, but they are not without their setbacks. For example, non-monotone discretizations like the finite element method may result in spurious oscillations with high Péclet numbers. Other common issues that may occur within highly heterogeneous and anisotropic diffusion-type equations are violations of the maximum principle and the non-negative constraint [Ciarlet and Raviart, 1973; Lipnikov et al., 2007; Liska and Shashkov, 2008; Genty and Potier, 2011]. Such numerical setbacks can result in algorithmic failures or sharp fronts that may result in erroneous approximations of reactive transport. Moreover, several important applications which require accurate predictive capabilities of transport solvers are often large-scale and cannot be solved on a single computer. It is important for numerical algorithms to not only ensure maximum principle but scale well with respect to both problem size and computing concurrency. Obtaining numerical solutions within a reasonable amount of time is the ultimate goal when selecting or designing algorithms that are robust and can ensure non-negative concentrations for a wide range of subsurface transport applications.

Before we discuss the prior works, it is important to discuss the reason behind the negative solutions under the conventional methods. The precise mathematical answer is that the conventional methods do not converge uniformly to the exact solution, which will be the case under strong 
anisotropic dispersion. In fact, the famous paper by [Ciarlet and Raviart, 1973] also provides the same reason in their study on discrete maximum principles of the Poisson's equation. The uniform convergence issues also manifest in the case of steep gradients and one such case is the famous Gibbs phenomenon [Gibbs, 1898, 1899]. It is difficult to explain intuitively why the Gibbs phenomenon occurs or why some sequence of functions do not converge uniformly. It is just the nature of the approximation of functions.

1.1. Prior works on non-negative formulations. The prior non-negative formulations can be broadly classified into the following five categories:

(a) Reporting the violations: In [Payette et al., 2012], several cases of violations of the maximum principle and the non-negative constraint have been showcased for different anisotropic diffusivity tensors. This paper also demonstrates that $h$ - and $p$-refinements do not eliminate these violations. The adverse effects due to violations of the non-negative constraint for non-linear ecological models and chemically reacting flows have been illustrated in [Mudunuru et al., 2015]. These mentioned papers and the references therein have clearly documented that the violations of the non-negative constraint need not be small, which is especially true in the case of anisotropic diffusion. However, neither of these papers have provided any fix to overcome these violations.

(b) Mesh restrictions: The first work on maximum principles under the finite element method can be traced back to the seminal paper by [Ciarlet and Raviart, 1973]. This paper considered isotropic diffusion, and has shown that an acute-angled triangular mesh (which is a restriction on the mesh) will satisfy the maximum principle under the finite element method. Anisotropic diffusion equations have been addressed in [Huang and Wang, 2015], wherein they developed an algorithm to generate metric-based meshes to satisfy the maximum principle for such equations. [Mudunuru and Nakshatrala, 2016b] have addressed various versions of maximum principles for diffusion and advection-diffusion equations and studied the performance of metric-based meshes for these equations. This paper highlighted the main deficiency of metric-based meshes, which is the need to alter the mesh for different diffusivity tensors. A comprehensive list and discussion of other prior works related to enforcing mesh restrictions to meet the maximum principle and the non-negative constraint can also be found in [Mudunuru and Nakshatrala, 2016b].

(c) Developing or altering formulations in the continuum setting: Two works that fall under this category are [Harari, 2004; Pal et al., 2016], both of which addressed transient transport problems. [Harari, 2004] utilized a stabilized method that is available for Helmholtz-type equations to construct a stabilized formulation for transient isotropic diffusion equations to meet the maximum principle. This approach, as presented in [Harari, 2004], is applicable to one-dimensional setting. [Pal et al., 2016] meets maximum principles for transient transport equations by employing two techniques. They rewrote transient transport equations, which are parabolic in nature, into modified Maxwell-Cattaneo equations, which are hyperbolic in nature, and employed the space-time Discontinuous Galerkin approach.

(d) Non-finite element approaches: A finite volume-based approach, to enforce the non-negative constraint, as proposed in [Potier, 2005], involves a non-linear iterative procedure to select appropriate collocation points for cell concentrations. This technique has been refined by several others including [Lipnikov et al., 2007] and [Sheng and Yuan, 2016]. Other similar approaches include the mimetic finite difference method [da Veiga et al., 2014], which ensures 
monotonicity and positivity. Since neither the finite difference nor finite volume methods are based on weak formulations, these mentioned works cannot be trivially extended to the finite element method.

(e) Optimization-based techniques at the discrete level: Several studies over the years [Nakshatrala and Valocchi, 2009; Nagarajan and Nakshatrala, 2011; Nakshatrala et al., 2013, 2016] have focused on the development of optimization-based methodologies that enforce the maximum principle and the non-negative constraint for diffusion problems. An optimization-based methodology based on the work of the aforementioned studies has been applied to enforce maximum principles advection-diffusion equations [Mudunuru and Nakshatrala, 2016a]. By reformulating the advection-diffusion problem as a mixed finite element formulation under the least-squares formalism, one introduces flux variables into the problem. The discrete formulation is also symmetric and positive-definite, so one can easily apply both bounded constraints and equality constraints to ensure non-negative solutions and local mass conservation respectively. It should be noted that one may also employ normal equations or the least-squares approach to ensure that the minimization problem for non-symmetric problems is convex [Demmel, 1997; Burdakov et al., 2012; Pal et al., 2016; Chang et al., 2016]. All of these studies have employed quadratic programming (QP) techniques to enforce the maximum principle on 2D academic problems, but the problems studied are small-scale and do not require state-of-the-art Krylov subspace (KSP) iterative solvers and preconditioners. Moreover, it is difficult to find solvers for least-squares or penalty-type problems due to the condition numbering of large systems of equations [Pyzara et al., 2011]. To this end, we are interested in numerical formulations and solvers that are suitable for large-scale applications. It has been shown recently that parallel optimization-based solvers can handle large-scale heterogeneous and anisotropic diffusion in [Chang et al., 2017], so we want to extend the work presented in that study to advection-diffusion equations.

1.2. Our approach and its salient features. The main contribution of this paper is to present a finite element computational framework applicable to both diffusion and advectiondiffusion equations that meets the maximum principle and satisfies the non-negative constraint. The framework is built by rewriting the weak formulation (WF) as a variational inequality (VI) [Ulbrich, 2011].

The field of VIs grew from a problem posed by Antonio Signorini [Signorini, 1933, 1959]. This problem was later coined as "Signorini problem" by Gaetano Fichera, who was a student of Signorini. Fichera posed the problem more precisely and obtained a variational inequality corresponding to the problem using which he established existence and uniqueness of solutions [Fichera, 1964, 1965]. VIs have been employed to study contact problems [Kikuchi and Oden, 1988; Hlavacek et al., 2012], obstacle problems [Rodrigues, 1987], elastoplastic problems [Hlavacek et al., 2012; Han and Reddy, 2012] and other problems arising in mechanics and mathematics [Kinderlehrer and Stampacchia, 2000]. If the bilinear form under the WF is symmetric, one can rewrite the WF as a QP, which is a special case of VIs [Chipot, 2009]. Most of the existing single-field WFs for advection-diffusion equations do not have symmetric bilinear forms, and hence one cannot construct equivalent problems under QP. To the best of the authors' knowledge, VIs have not be employed to develop numerical formulations to satisfy maximum principles and the non-negative constraint for anisotropic advection-diffusion equations. 
The framework is particularly suited for large-scale problems, which is the case with many practical subsurface applications. The proposed framework enjoys the following salient features:

(I) One can enforce bounded constraints for any transport problems that may be non-symmetric or nonlinear.

(II) One can employ any numerical formulation, even a single-field formulation, for solving advection-diffusion equations.

(III) One can leverage on existing high performance computing libraries and toolkits (e.g., solvers and preconditioners).

(IV) The framework is amenable for parallel computing, which will be illustrated using both strong and weak scaling studies.

The rest of the paper is organized as follows. In Section 2, we present the boundary value problem for steady-state diffusion and advection-diffusion equations. In Section 3, we present the variational inequality (VI) and the various single-field weak formulations (WF) in the continuous setting. In Section 4, we propose the computational framework in a discrete setting and discuss in detail the specific solvers and implementation procedure. In Section 5, numerical results for the steady-state governing equations under the proposed framework are shown, and we conduct a thorough strong and weak-scaling study to demonstrate the parallel performance. In Section 6 , we provide an extension of the proposed framework to transient problems and illustrate the performance of miscible displacement in porous media, which is a coupled non-linear phenomenon. Conclusions are drawn in Section 7. To facilitate the reader to be able to reproduce the results given in this paper, sample Firedrake project files along with the discussion on the solution strategy for large-scale Darcy equations are provided in Appendices A and B.

On the notational front, we denote all the continuum vectors by lowercase boldface unitalicized letters, and the vectors in the discrete setting are denoted by lowercase boldface italic letters. We denote all the continuum second-order tensors by boldface uppercase unitalicized letters, and all the finite element matrices are denoted by uppercase boldface italicized letters. In this paper, repeated indices do not imply summation. Other notational conventions are introduced as needed.

\section{GOVERNING EQUATIONS IN THE CONTINUOUS SETTING}

Let $\Omega \subset \mathbb{R}^{d}$ be a bounded open domain, where ' $d$ ' denotes the number of spatial dimensions. The boundary is denoted by $\partial \Omega=\bar{\Omega}-\Omega$, where a superposed bar denotes the set closure. We denote the set of all $k$-times continuously differentiable functions on $\Omega$ by $C^{k}(\Omega)$. We denote the set of all functions in $C^{0}(\Omega)$ that are continuous to the boundary by $C^{0}(\bar{\Omega})$. A spatial point is denoted by $\mathbf{x} \in \bar{\Omega}$. The gradient and divergence operators with respect to $\mathbf{x}$ are, respectively, denoted by $\operatorname{grad}[\cdot]$ and $\operatorname{div}[\cdot]$. The unit outward normal to boundary is denoted by $\widehat{\mathbf{n}}(\mathbf{x})$.

Let $c(\mathbf{x})$ denote the concentration field. The boundary is divided into two parts: $\Gamma^{\mathrm{D}}$ and $\Gamma^{\mathrm{N}}$, such that $\Gamma^{\mathrm{D}} \cup \Gamma^{\mathrm{N}}=\partial \Omega$ and $\Gamma^{\mathrm{D}} \cap \Gamma^{\mathrm{N}}=\emptyset . \Gamma^{\mathrm{D}}$ is that part of the boundary on which Dirichlet boundary conditions are enforced (i.e., concentration is prescribed). $\Gamma^{\mathrm{N}}$ is the part of the boundary on which Neumann boundary conditions are enforced (i.e., flux is prescribed). When advection is present, the Neumann boundary is further divided into inflow and outflow regions, which are defined as follows:

$$
\begin{array}{r|r}
\Gamma_{\text {inflow }}^{\mathrm{N}}:=\left\{\mathbf{x} \in \Gamma^{\mathrm{N}}\right. & \mathbf{v}(\mathbf{x}) \cdot \widehat{\mathbf{n}}(\mathbf{x})<0\} \\
\Gamma_{\text {outflow }}^{\mathrm{N}}:=\left\{\begin{array}{l|l}
\mathbf{x} \in \Gamma^{\mathrm{N}} & \mathbf{v}(\mathbf{x}) \cdot \widehat{\mathbf{n}}(\mathbf{x}) \geq 0\}
\end{array}\right.
\end{array}
$$


For uniqueness of the solution under a steady-state response, we assume that concentration is prescribed on a non-zero part of the boundary (i.e., meas $\left(\Gamma^{\mathrm{D}}\right)>0$ ).

2.1. Strong problems (SP). The strong problem (SP) for steady-state diffusion reads: Find $c(\mathbf{x}) \in C^{2}(\Omega) \cap C^{0}(\bar{\Omega})$ such that we have

$$
\begin{array}{cl}
-\operatorname{div}[\mathbf{D}(\mathbf{x}) \operatorname{grad}[c(\mathbf{x})]]=f(\mathbf{x}) & \text { in } \Omega \\
c(\mathbf{x})=c^{\mathrm{P}}(\mathbf{x}) & \text { on } \Gamma^{\mathrm{D}} \\
-\widehat{\mathbf{n}}(\mathbf{x}) \cdot \mathbf{D}(\mathbf{x}) \operatorname{grad}[c(\mathbf{x})]=q^{\mathrm{p}}(\mathbf{x}) & \text { on } \Gamma^{\mathrm{N}}
\end{array}
$$

and the SP for steady-state advection-diffusion reads: Find $c(\mathbf{x}) \in C^{2}(\Omega) \cap C^{0}(\bar{\Omega})$ such that we have

$$
\begin{array}{cl}
\mathbf{v}(\mathbf{x}) \cdot \operatorname{grad}[c(\mathbf{x})]-\operatorname{div}[\mathbf{D}(\mathbf{x}) \operatorname{grad}[c(\mathbf{x})]]=f(\mathbf{x}) & \text { in } \Omega \\
c(\mathbf{x})=c^{\mathrm{p}}(\mathbf{x}) & \text { on } \Gamma^{\mathrm{D}} \\
\widehat{\mathbf{n}}(\mathbf{x}) \cdot(\mathbf{v}(\mathbf{x}) c(\mathbf{x})-\mathbf{D}(\mathbf{x}) \operatorname{grad}[c(\mathbf{x})])=q^{\mathrm{p}}(\mathbf{x}) & \text { on } \Gamma_{\text {inflow }}^{\mathrm{N}} \\
-\widehat{\mathbf{n}}(\mathbf{x}) \cdot \mathbf{D}(\mathbf{x}) \operatorname{grad}[c(\mathbf{x})]=q^{\mathrm{p}}(\mathbf{x}) & \text { on } \Gamma_{\text {outflow }}^{\mathrm{N}}
\end{array}
$$

where $\mathbf{v}(\mathbf{x})$ is the advective velocity, $f(\mathbf{x})$ is the prescribed volumetric source/sink, $c^{\mathrm{p}}(\mathbf{x})$ is the prescribed concentration on the boundary, $q^{\mathrm{P}}(\mathbf{x})$ is the prescribed flux on the boundary, and $\mathbf{D}(\mathbf{x})$ is the second-order diffusivity tensor. The diffusivity tensor is assumed to be bounded and uniformly elliptic. That is, there exist two constants $0<\xi_{1} \leq \xi_{2}<+\infty$ such that

$$
\xi_{1} \mathbf{y} \cdot \mathbf{y} \leq \mathbf{y} \cdot \mathbf{D}(\mathbf{x}) \mathbf{y} \leq \xi_{2} \mathbf{y} \cdot \mathbf{y} \quad \forall \mathbf{y} \in \mathbb{R}^{d}
$$

Moreover, the diffusivity tensor is assumed to be symmetric. That is,

$$
\mathbf{D}(\mathbf{x})=\mathbf{D}^{\mathrm{T}}(\mathbf{x}) \quad \forall \mathbf{x} \in \Omega
$$

A solution to SP is commonly referred to as a classical solution.

2.2. Maximum principle and the non-negative constraint. From the theory of partial differential equations, it is well-known that a classical solution for the above mentioned SPs satisfies maximum principles. For completeness, we provide below the statement of the classical maximum principle of second-order elliptic partial differential equations with Dirichlet boundary conditions on the entire boundary.

Theorem 1. (Classical maximum principle) If $\Gamma^{\mathrm{D}}=\partial \Omega, c(\mathbf{x}) \in C^{2}(\Omega) \cap C^{0}(\bar{\Omega})$ and $f(\mathbf{x}) \leq 0$, then

$$
\max _{\mathbf{x} \in \Omega} c(\mathbf{x}) \leq \max _{\mathbf{x} \in \partial \Omega} c^{\mathrm{p}}(\mathbf{x})
$$

Proof. A proof can be found in [Gilbarg and Trudinger, 2001].

A generalization of the classical maximum principle that is relevant to this paper is provided in [Mudunuru and Nakshatrala, 2016a]. Specifically, they have extended the classical maximum principle on four fronts: the regularity of the solution is relaxed to $C^{1}(\Omega) \cap C^{0}(\bar{\Omega})$, the regularity of the volumetric source $f(\mathbf{x})$ is relaxed to the space of square integrable functions, the boundary can have both Dirichlet and Neumann boundary conditions, and the Neumann boundary conditions are further divided into inflow and outflow (i.e., similar to equations (2.1a)-(2.1b)). For the sake of brevity, we defer all interested readers to the suggested reference. Another property that is relevant 


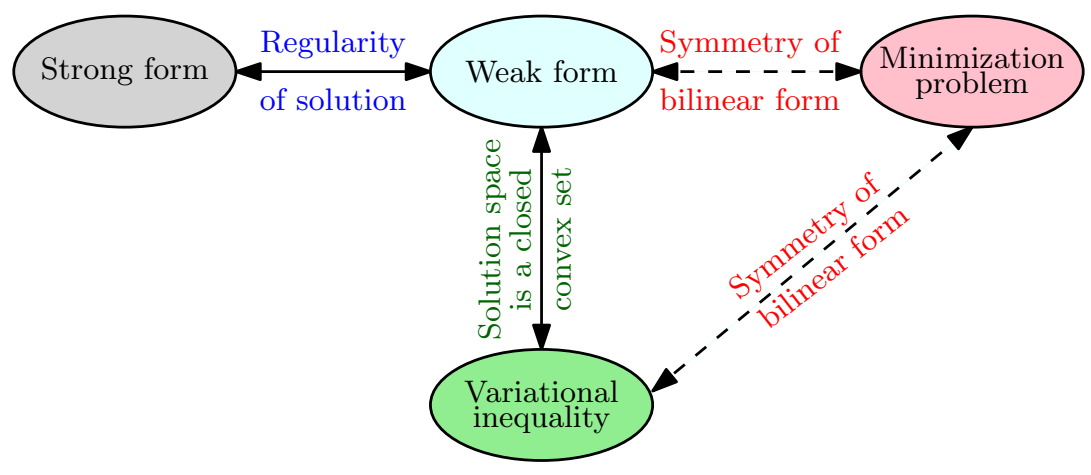

FigURE 1. Relationships between the strong problem (SP), a weak formulation (WF), a variational inequality (VI), and the minimization problem (MP).

to this paper is non-negative solutions, which can be shown to be a special case of maximum principles. In particular, the above maximum principle implies the following result:

Corollary 1. (Non-negative solutions) If $\Gamma^{\mathrm{D}}=\partial \Omega, c(\mathbf{x}) \in C^{2}(\Omega) \cap C^{0}(\bar{\Omega}), c^{\mathrm{p}}(\mathbf{x}) \geq 0$, and $f(\mathbf{x}) \geq 0$, then

$$
0 \leq c(\mathbf{x}) \quad \forall \mathbf{x} \in \bar{\Omega}
$$

The central aim of this paper is to obtain numerical solutions to the above governing equations (i.e., equations (2.2) and (2.3)) that respect maximum principles and the non-negative constraint.

The main task will then be to find an appropriate setting for numerical solutions. The finite difference method directly discretizes the SP. However, under the finite element method, the SP is rewritten as a $\mathrm{WF}$, which is equivalent to the $\mathrm{SP}$ under some regularity assumptions. A solution to a WF is referred to as a weak solution. As mentioned in Section 1 and will be shown using several examples later in this paper, a WF does not guarantee non-negative solutions in the discrete setting. To overcome this deficiency, some non-negative formulations, especially for diffusion-type equations, have rewritten the WF as an equivalent minimization problem (MP) and augmented with bound constraints. However, it needs to be emphasized that such conversion is not always possible, which is the case with the typical WF for advection-diffusion equations, as these formulations have non-symmetric bilinear forms. In order to handle non-self-adjoint differential operators (e.g., advection-diffusion equation) and WFs with non-symmetric bilinear forms, we rewrite a given WF as a VI. In order to satisfy maximum principles and the non-negative constraint, we restrict the feasible solution space of the VI formulation using bound constraints. It needs to be mentioned that one can pose the VI as an equivalent MP only if the bilinear form is symmetric. Figure 1 illustrates the various ways of rewriting the governing equations, and the conditions under which one form is equivalent to the other. We now present various WFs for diffusion and advection-diffusion equations, which will form the basis for our proposed VI-based formulations.

\section{VARIATIONAL INEQUALITIES AND WEAK FORMULATIONS}

The non-negative constraint and maximum principles restrict the feasible solution space to a closed convex set. A variational inequality (VI) is basically a variational problem on a convex set, which need not be a vector space. To this end, let $\mathcal{C}$ denote the solution space for the concentration 
field, and $\mathcal{K}$ be a closed convex subset of $\mathcal{C}$. The subset $\mathcal{K}$ is defined by the underlying maximum principles and the non-negative constraint. The formulation based on VIs corresponding to the mentioned SPs can be compactly written as: Find $c(\mathbf{x}) \in \mathcal{K}$ such that we have

$$
\mathcal{B}(w-c ; c) \geq \mathcal{L}(w-c) \quad \forall w(\mathbf{x}) \in \mathcal{K}
$$

where $\mathcal{B}(\cdot ; \cdot)$ is a bilinear form and $\mathcal{L}(\cdot)$ is a linear functional, whose specific choices are provided by the associated weak formulation. A WF can be abstractly written as: Find $c(\mathbf{x}) \in \mathcal{C}$ such that we have

$$
\mathcal{B}(w ; c)=\mathcal{L}(w) \quad \forall w(\mathbf{x}) \in \mathcal{W}
$$

where $\mathcal{C}$ and $\mathcal{W}$ are appropriate function spaces for a given WF. Our intention is to illustrate the applicability of the proposed VI framework to a variety of WFs. To this end, we employ the continuous Galerkin (GAL), Streamlined Upwind Petrov-Galerkin (SUPG), and Discontinuous Galerkin (DG) formulations, which are documented below. For convenience, the standard $L_{2}$ inner-product over $K$ is denoted as follows:

$$
(a ; b)_{K}=\int_{K} a(\mathbf{x}) \cdot b(\mathbf{x}) \mathrm{d} K
$$

3.1. Continuous Galerkin. The relevant function spaces are:

$$
\begin{aligned}
\mathcal{C} & :=\left\{c(\mathbf{x}) \in H^{1}(\Omega) \mid c(\mathbf{x})=c^{\mathrm{p}}(\mathbf{x}) \text { on } \Gamma^{\mathrm{D}}\right\} \\
\mathcal{W} & :=\left\{w(\mathbf{x}) \in H^{1}(\Omega) \mid w(\mathbf{x})=0 \text { on } \Gamma^{\mathrm{D}}\right\}
\end{aligned}
$$

where $H^{1}(\Omega)$ is a Sobolev space [Brezis, 2010]. We assume that $f(\mathbf{x}) \in H^{-1}(\Omega)$, which is a dual space corresponding to $H^{1}(\Omega)$. We employ the GAL formulation for the diffusion problem, for which the bilinear form and linear functional are:

$$
\begin{aligned}
& \mathcal{B}_{\mathrm{GAL}}(w ; c):=(\operatorname{grad}[w(\mathbf{x})] ; \mathbf{D}(\mathbf{x}) \operatorname{grad}[c(\mathbf{x})])_{\Omega} \\
& \mathcal{L}_{\mathrm{GAL}}(w):=(w(\mathbf{x}) ; f(\mathbf{x}))_{\Omega}-\left(w(\mathbf{x}) ; q^{\mathrm{p}}(\mathbf{x})\right)_{\Gamma^{\mathrm{N}}}
\end{aligned}
$$

For the advection-diffusion problem, spurious oscillations may arise under the GAL formulation for high Péclet numbers. Herein, we employ the SUPG formulation [Brooks and Hughes, 1982], and the corresponding bilinear form and linear functional are:

$$
\begin{aligned}
& \mathcal{B}_{\mathrm{SUPG}}(w ; c):=\mathcal{B}_{\mathrm{RES}}(w ; c)+(w(\mathbf{x}) ; \mathbf{v}(\mathbf{x}) \cdot \operatorname{grad}[c(\mathbf{x})])_{\Omega}+(\operatorname{grad}[w(\mathbf{x})] ; \mathbf{D}(\mathbf{x}) \operatorname{grad}[c(\mathbf{x})])_{\Omega} \\
& \mathcal{L}_{\mathrm{SUPG}}(w):=\mathcal{L}_{\mathrm{RES}}(w)+(w(\mathbf{x}) ; f(\mathbf{x}))_{\Omega}-\left(w(\mathbf{x}) ; q^{\mathrm{p}}(\mathbf{x})\right)_{\Gamma^{\mathrm{N}}}
\end{aligned}
$$

where the residual terms

$$
\begin{aligned}
& \mathcal{B}_{\mathrm{RES}}(w ; c):=\left(\frac{h}{2\|\mathbf{v}(\mathbf{x})\|} \mathbf{v}(\mathrm{x}) \cdot \operatorname{grad}[w(\mathbf{x})] ; \mathbf{v}(\mathrm{x}) \cdot \operatorname{grad}[c(\mathbf{x})]-\operatorname{div}[\mathbf{D}(\mathbf{x}) \operatorname{grad}[c(\mathbf{x})]]\right)_{\Omega} \\
& \mathcal{L}_{\mathrm{RES}}(w):=\left(\frac{h}{2\|\mathbf{v}(\mathrm{x})\|} \mathbf{v}(\mathrm{x}) \cdot \operatorname{grad}[w(\mathbf{x})] ; f(\mathbf{x})\right)_{\Omega}
\end{aligned}
$$

and $h$ denotes the element-wise diameter. 
3.2. Discontinuous Galerkin. For several transport applications, it is highly desirable to possess element-wise mass balance property, as it is an important fundamental physical law [Turner et al., 2011]. This is particularly true when the transport is coupled with chemical reactions and biofilm growth [Werth and Valocchi, 2005; von der Schulenburg et al., 2009]. The GAL and SUPG formulations do not possess this property without any further modification or enrichment to their formulations. One way to ensure this property under a single-field finite element framework is through the use of the DG formulations (see [Arnold et al., 2002; Riviére and Wheeler, 2002; Cockburn, 2003; Li and Riviére, 2015b,a, 2016; Pal et al., 2016] and the references within for further details). To present the DG formulation employed in the paper, we now introduce relevant notation.

The domain $\Omega$ is divided into $S$ subdomains:

$$
\Omega=\bigcup_{i=1}^{S} \omega_{i}
$$

The boundary of the subdomain $\omega_{i}$ is denoted by $\partial \omega_{i}$. The interior face between $\omega_{i}$ and $\omega_{j}$ is denoted by $\Gamma_{i j}$. That is,

$$
\Gamma_{i j}=\partial \omega_{i} \cap \partial \omega_{j}
$$

The set of all points on the interior faces is denoted by $\Gamma_{\text {int }}$. Mathematically,

$$
\Gamma_{\text {int }}=\bigcup_{i=1, i<j}^{S} \Gamma_{i j}
$$

For an interior face, we denote the subdomains shared by this face by $\omega^{+}$and $\omega^{-}$. The outward normals on this face for these subdomains are, respectively, denoted by $\widehat{\mathbf{n}}^{+}$and $\widehat{\mathbf{n}}^{-}$. Employing Brezzi's notation [Arnold et al., 2002], the average and jump operators on an interior face are defined as follows:

$$
\{c\}:=\frac{c^{+}+c^{-}}{2} \text { and } \quad[[c]]:=c^{+} \widehat{\mathbf{n}}^{+}+c^{-} \widehat{\mathbf{n}}^{-}
$$

where

$$
c^{+}=\left.c\right|_{\partial \omega^{+}} \quad \text { and } \quad c^{-}=\left.c\right|_{\partial \omega^{-}}
$$

One of the most popular DG formulations is the Interior Penalty method, which for equation $(2.2)$ is written as:

$$
\begin{aligned}
\mathcal{B}_{\mathrm{DG}}(w ; c) & :=(\operatorname{grad}[w(\mathbf{x})] ; \mathbf{D}(\mathbf{x}) \operatorname{grad}[c(\mathbf{x})])_{\Omega}-([[w(\mathbf{x})]] ;\{\mathbf{D}(\mathbf{x}) \operatorname{grad}[c(\mathbf{x})]\})_{\Gamma_{\text {int }}} \\
& +\epsilon(\{\mathbf{D}(\mathbf{x}) \operatorname{grad}[w(\mathbf{x})]\} ;[[c(\mathbf{x})]])_{\Gamma_{\text {int }}}+\frac{\gamma}{h}([[w(\mathbf{x})]] ;[[c(\mathbf{x})]])_{\Gamma_{\text {int }}} \\
\mathcal{L}_{\mathrm{DG}}(w) & :=(w(\mathbf{x}) ; f(\mathbf{x}))_{\Omega}-\left(w(\mathbf{x}) ; q^{\mathrm{P}}(\mathbf{x})\right)_{\Gamma^{\mathrm{N}}}
\end{aligned}
$$

where the penalty term $\gamma=2 \frac{(d+1)}{d}$ [Shahbazi, 2005] for first-order elements and $\epsilon \in\{-1,0,1\}$ denotes the Symmetric, Incomplete, and Non-symmetric Interior Penalty methods respectively. For equation (2.3), the DG formulation can be written as:

$$
\begin{gathered}
\mathcal{B}_{\mathrm{DG}}(w ; c):=(\operatorname{grad}[w(\mathbf{x})] ; \mathbf{D}(\mathbf{x}) \operatorname{grad}[c(\mathbf{x})])_{\Omega}-([[w(\mathbf{x})]] ;\{\mathbf{D}(\mathbf{x}) \operatorname{grad}[c(\mathbf{x})]\})_{\Gamma_{\mathrm{int}}} \\
+\epsilon(\{\mathbf{D}(\mathbf{x}) \operatorname{grad}[w(\mathbf{x})]\} ;[[c(\mathbf{x})]])_{\Gamma_{\mathrm{int}}}+\frac{\gamma}{h}([[w(\mathbf{x})]] ;[[c(\mathbf{x})]])_{\Gamma_{\mathrm{int}}}
\end{gathered}
$$




$$
\begin{aligned}
& (w(\mathbf{x}) ; \mathbf{v}(\mathbf{x}) \cdot \operatorname{grad}[c(\mathbf{x})])_{\Omega}-\left([[w(\mathbf{x})]] ; c^{\mathrm{up}}(\mathbf{x}) \mathbf{v}(\mathbf{x})\right)_{\Gamma_{\text {int }}} \\
\mathcal{L}_{\mathrm{DG}}(w) & :=(w(\mathbf{x}) ; f(\mathbf{x}))_{\Omega}-\left(w(\mathbf{x}) ; q^{\mathrm{p}}(\mathbf{x})\right)_{\Gamma^{\mathrm{N}}}
\end{aligned}
$$

where the upwinding term $c^{\mathrm{up}}(\mathbf{x})$ is defined as:

$$
c^{\mathrm{up}}(\mathbf{x})= \begin{cases}c^{+}(\mathbf{x}) & \text { if } \mathbf{v}(\mathbf{x}) \cdot \widehat{\mathbf{n}}^{+}(\mathbf{x})>0 \\ c^{-}(\mathbf{x}) & \text { otherwise }\end{cases}
$$

For the remainder of this paper, we shall consider only the Symmetric Interior Penalty method where $\epsilon=-1$.

3.3. A theoretical discussion. The bilinear form is assumed to be continuous (i.e., bounded above). That is, there exists a constant $\kappa_{1}>0$ such that

$$
\mathcal{B}(w ; c) \leq \kappa_{1}\|c\|\|w\| \quad \forall c(\mathbf{x}), w(\mathbf{x}) \in \mathcal{C}
$$

In addition, the bilinear form is assumed to be coercive. That is, there exists a constant $\kappa_{2}>0$ such that

$$
\kappa_{2}\|c\|^{2} \leq \mathcal{B}(c ; c) \quad \forall c(\mathbf{x}) \in \mathcal{C}
$$

Recall that $\mathcal{L}(\cdot)$ is assumed to be a linear continuous functional on $\mathcal{C}$. Then, from the Lax-Milgram theorem [Brenner and Scott, 1994], it is known that a unique solution exists under the WF. Under the same conditions on the bilinear form and the linear functional, a unique solution exists for the associated VI if $\mathcal{K} \subset \mathcal{C}$ is a closed convex subset [Lions and Stampacchia, 1967]. A solution of the $\mathrm{VI}$ is a solution of the $\mathrm{WF}$ if $\mathcal{C}=\mathcal{K}$. Moreover, if the bilinear form is symmetric, that is,

$$
\mathcal{B}(w ; c)=B(c ; w)
$$

then the WF and the VI are equivalent to the following MP: Find $c(\mathbf{x}) \in \mathcal{C}$ such that

$$
\underset{c(\mathbf{x}) \in \mathcal{C}}{\operatorname{minimize}} \frac{1}{2} \mathcal{B}(c ; c)-\mathcal{L}(c)
$$

These relations are pictorially described in Figure 1. From a theoretical point of view, it is important to note that the VIs that we will be dealing with for steady-state problems will be elliptic of first kind. For further details on infinite-dimensional VIs, see [Glowinski, 1984; Duvaut and Lions, 1976].

\section{PROPOSED COMPUTATIONAL FRAMEWORK IN A DISCRETE SETTING}

We denote the total number of degrees-of-freedom by "ndofs". We also denote the vector of ones by $\mathbf{1}$, whose size will be apparent from the context in which it is used. The component-wise inequalities are denoted by $\preceq$ and $\succeq$. That is,

$$
\begin{aligned}
& \boldsymbol{a} \preceq \boldsymbol{b} \quad \text { implies that } \quad a_{i} \leq b_{i} \forall i \\
& \boldsymbol{a} \succeq \boldsymbol{b} \quad \text { implies that } \quad a_{i} \geq b_{i} \forall i
\end{aligned}
$$

The vector of unknown nodal concentrations is denoted by $\boldsymbol{c}$, and the corresponding nodal source vector is denoted by $\boldsymbol{f}$. The coefficient matrix after a finite element discretization is denoted by $\boldsymbol{K}$. 
Note that the vectors $\boldsymbol{c}$ and $\boldsymbol{f}$ are of size $n d o f s \times 1$, and the matrix $\boldsymbol{K}$ is of size $n d o f s \times n d o f s$. We denote the standard inner-product in Euclidean spaces by $\langle\cdot ; \cdot\rangle$. That is,

$$
\langle\boldsymbol{a} ; \boldsymbol{b}\rangle=\sum_{i}^{n d o f s} a_{i} b_{i} \quad \forall \boldsymbol{a}, \boldsymbol{b} \in \mathbb{R}^{n d o f s}
$$

The formulation in the discrete setting will be posed as a mixed complementarity problem (MCP) [Kinderlehrer and Stampacchia, 2000]. For convenience, we define $\boldsymbol{h} \in \mathbb{R}^{\text {ndofs }}$ as:

$$
h:=K c-f
$$

The corresponding MCP reads: Find $c_{\min } \mathbf{1} \preceq \boldsymbol{c} \preceq c_{\max } \mathbf{1}$ such that for each $i \in\{1, \ldots, n d o f s\}$

$$
\begin{array}{ll}
h_{i}(\boldsymbol{c}) \geq 0 & \text { if } c_{\min }=c_{i} \\
h_{i}(\boldsymbol{c})=0 & \text { if } c_{\min } \leq c_{i} \leq c_{\max } \\
h_{i}(\boldsymbol{c}) \leq 0 & \text { if } c_{i}=c_{\max }
\end{array}
$$

where $c_{\min }$ and $c_{\max }$, respectively, denote the minimum and maximum concentrations, which are provided by the maximum principle or the non-negative constraint. Simple complementarity conditions arise from the first-order optimality conditions in optimization. For bound-constrained optimization, $\boldsymbol{h}$ corresponds to the gradient of the objective functional. If one has only the nonnegative constraints (i.e., $c_{\min }=0$ and $c_{\max }=+\infty$ ), then the problem reduces to a non-linear complementarity problem, which is a special case of MCP. For details on non-linear complementarity problems, see [Facchinei and Pang, 2003]. Note that the feasible region, which is restricted by the bound constraints, form a parallelepiped, which is a convex set [Boyd and Vandenberghe, 2004].

Let the feasible region $\mathcal{K}$ be a convex subset of $\mathbb{R}^{\text {ndofs }}$. In our case, the feasible region is restricted by constraints which are in the form of finite number of linear equalities and inequalities. This makes the feasible region to be a polyhedron, which is a convex set [Boyd and Vandenberghe, 2004]. It should be noted that bound constraints are a special case of linear inequalities. With this machinery at our disposal, one can pose the second formulation based on variational inequalities, which reads: Find $c \in \mathcal{K}$ such that we have

$$
\langle\boldsymbol{K} \boldsymbol{c} ; \boldsymbol{v}-\boldsymbol{c}\rangle \geq\langle\boldsymbol{f} ; \boldsymbol{v}-\boldsymbol{c}\rangle \quad \forall \boldsymbol{v} \in \mathcal{K}
$$

Note that MCP is a special case of VIs in which the feasible region is a parallelepiped (i.e., one has only bound constraints). The conditions under which a solution exists for the finite-dimensional VI given in equation (4.5) is pictorially described in Figure 2.

If the coefficient matrix $\boldsymbol{K}$ is symmetric, one can alternatively enforce maximum principles and the non-negative constraint using QP, which has been illustrated in [Nagarajan and Nakshatrala, 2011; Nakshatrala et al., 2016] for small-scale problems, and in [Chang et al., 2017] for largescale problems in parallel environments. Therefore, this approach is only applicable for formally self-adjoint differential operators. The formulation can be posed as follows:

$$
\begin{array}{cl}
\underset{\boldsymbol{c} \in \mathbb{R}^{\text {ndofs }}}{\operatorname{minimize}} & \frac{1}{2}\langle\boldsymbol{c} ; \boldsymbol{K} \boldsymbol{c}\rangle-\langle\boldsymbol{c} ; \boldsymbol{f}\rangle \\
\text { subject to } & c_{\min } \mathbf{1} \preceq \boldsymbol{c} \preceq c_{\max } \mathbf{1}
\end{array}
$$




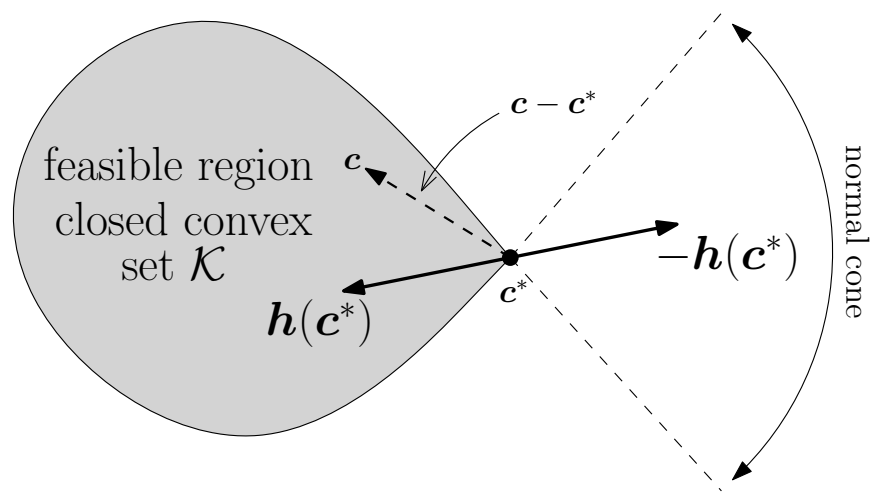

FiguRE 2. This figure illustrates the condition under which a solution exists for a variational inequality of the form $\langle\boldsymbol{h}(\boldsymbol{c}), \tilde{\boldsymbol{c}}-\boldsymbol{c}\rangle \geq 0 \forall \tilde{\boldsymbol{c}} \in \mathcal{K}$. Here, $\boldsymbol{c}^{*}$ denotes a solution of the VI. The normal cone of $\mathcal{K}$ at $\boldsymbol{c}^{*}$ is defined as $\mathcal{N}\left(\boldsymbol{c}^{*}\right):=$ $\left\{\boldsymbol{w} \in \mathbb{R}^{\text {ndofs }} \mid\left\langle\boldsymbol{w} ; \boldsymbol{c}-\boldsymbol{c}^{*}\right\rangle \leq \mathbf{0} \forall \boldsymbol{c} \in \mathcal{K}\right\}$.

In addition, if $\boldsymbol{K}$ is positive-definite the objective function becomes convex. The resulting optimization problem then belongs to the special case of convex quadratic programming for which sophisticated solvers exist.

REMARK 1. It should be mentioned that a quick fix to eliminate negative violations is through the so-called clipping procedure. However, this procedure is rather ad hoc and, more importantly, it is not variationally consistent. On the other hand, the proposed VI-based computational framework not only ensures non-negative solutions but also has a firm variational basis. We will also illustrate that the solutions under the proposed framework need not necessarily match the solution under the clipping procedure.

4.1. Theoretical results in the discrete setting. In this paper, we are interested in problems with two different cases of bound constraints. In the first case, we have both lower and upper bounds. In the second case, we have only the lower bound. The lower bound typically comes from the non-negative constraint, and the upper bound comes from maximum principles. We now discuss existence results for finite-dimensional VIs under the mentioned two cases of bound constraints.

We begin by noting that the feasible set $\mathcal{K}$ will be convex and closed for both the sets of bound constraints. In the first case, the feasible set will also be bounded, which makes the feasible set to be compact (which, in the context of Euclidean spaces, is equivalent to closed and bounded). We therefore deal with both the cases separately.

THEOREM 2. (Existence based on compactness of $\mathcal{K}$ ) If $\mathcal{K}$ is compact and convex, then a solution exists to the finite-dimensional VI (4.5).

Proof. A proof can be constructed using the Brouwer's fixed point theorem and can be found in [Facchinei and Pang, 2003].

THEOREM 3. (Existence based on positive-definiteness of $\operatorname{sym}[\boldsymbol{K}]$ ) If the symmetric part of the coefficient matrix $\boldsymbol{K}$ (i.e., $\operatorname{sym}[\boldsymbol{K}]$ ) is positive-definite, a solution to the finite-dimensional VI (4.5) exists. (Note that the feasible set $\mathcal{K}$ need not be compact.) 


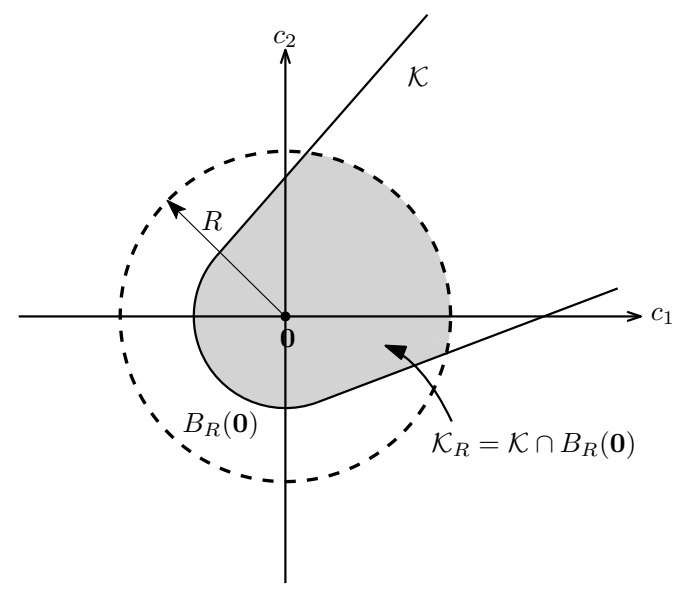

Figure 3. A pictorial description of $B_{R}(\mathbf{0})$ and $\mathcal{K}_{R}$. These sets are used in Theorem 4.

Proof. Let

$$
\boldsymbol{g}(\boldsymbol{c}):=\boldsymbol{K} \boldsymbol{c}-\boldsymbol{f}
$$

The VI then becomes

$$
\langle\boldsymbol{g}(\boldsymbol{c}) ; \widetilde{\boldsymbol{c}}-\boldsymbol{c}\rangle \geq 0 \quad \forall \widetilde{\boldsymbol{c}} \in \mathcal{K}
$$

Clearly, the function $\boldsymbol{g}(\boldsymbol{c})$ is continuous. Moreover, the function $\boldsymbol{g}(\boldsymbol{c})$ satisfies the following coercive condition:

$$
\frac{\langle\boldsymbol{g}(\boldsymbol{c})-\boldsymbol{g}(\widetilde{\boldsymbol{c}}) ; \boldsymbol{c}-\widetilde{\boldsymbol{c}}\rangle}{\|\boldsymbol{c}-\widetilde{\boldsymbol{c}}\|} \rightarrow \infty
$$

as $\|\boldsymbol{c}\| \rightarrow \infty$. To wit, since $\operatorname{sym}[\boldsymbol{K}]$ is positive-definite and symmetric, the minimum eigenvalue $\lambda_{\min }$ is real and positive. One can then write:

$$
\lambda_{\min }\|\boldsymbol{c}-\widetilde{\boldsymbol{c}}\|^{2} \leq(\boldsymbol{c}-\widetilde{\boldsymbol{c}}) \cdot \operatorname{sym}[\boldsymbol{K}](\boldsymbol{c}-\widetilde{\boldsymbol{c}})=(\boldsymbol{c}-\widetilde{\boldsymbol{c}}) \cdot \boldsymbol{K}(\boldsymbol{c}-\widetilde{\boldsymbol{c}})=\langle\boldsymbol{g}(\boldsymbol{c})-\boldsymbol{g}(\widetilde{\boldsymbol{c}}) ; \boldsymbol{c}-\widetilde{\boldsymbol{c}}\rangle
$$

That is,

$$
\lambda_{\min }\|\boldsymbol{c}-\widetilde{\boldsymbol{c}}\| \leq \frac{\langle\boldsymbol{g}(\boldsymbol{c})-\boldsymbol{g}(\widetilde{\boldsymbol{c}}) ; \boldsymbol{c}-\widetilde{\boldsymbol{c}}\rangle}{\|\boldsymbol{c}-\widetilde{\boldsymbol{c}}\|}
$$

We thus have shown that the function $\boldsymbol{g}(\boldsymbol{c})$ is continuous and coercive. Under such conditions, a solution exists to the VI (4.8) (e.g., see [Nagurney, 2002; Nagurney and Zhang, 2012]).

We next present another existence theorem which is particularly useful when the feasible set is unbounded (for example, when we have only one of the bounds - either lower or upper bounds). Let $B_{R}(\mathbf{0})$ is a hypersphere of radius $R$ centered at $\mathbf{0}$, and let $\mathcal{K}_{R}=\mathcal{K} \cap B_{R}(\mathbf{0})$ (see Figure 3). Clearly, $\mathcal{K}_{R}$ is bounded.

Theorem 4. (Existence based on $\mathcal{K}_{R}$ ) A solution exists to the $V I$ (4.5) on $\mathcal{K}$ (which need not be bounded) if and only if there exists $R>0$ and a solution $\boldsymbol{c}^{*} \in \mathcal{K}_{R}$ that satisfies the following VI:

$$
\left\langle\boldsymbol{K} \boldsymbol{c}^{*} ; \widetilde{\boldsymbol{c}}-\boldsymbol{c}^{*}\right\rangle \geq\left\langle f ; \widetilde{\boldsymbol{c}}-\boldsymbol{c}^{*}\right\rangle \quad \forall \widetilde{\boldsymbol{c}} \in \mathcal{K}_{R}
$$

which is defined on a bounded set.

Proof. See [Nagurney, 2002]. 
THEOREM 5. (Uniqueness) If the symmetric part of the coefficient matrix $\boldsymbol{K}$ is positive-definite, then the finite-dimensional VI (4.5) has a unique solution if it exists.

Proof. On the contrary, assume that $\boldsymbol{c}_{1}$ and $\boldsymbol{c}_{2}$ are two different solutions of the VI (4.5). This implies that

$$
\begin{array}{ll}
\left\langle\boldsymbol{K} \boldsymbol{c}_{1} ; \boldsymbol{v}-\boldsymbol{c}_{1}\right\rangle \geq\left\langle\boldsymbol{f} ; \boldsymbol{v}-\boldsymbol{c}_{1}\right\rangle & \forall \boldsymbol{v} \in \mathcal{K} \\
\left\langle\boldsymbol{K} \boldsymbol{c}_{2} ; \boldsymbol{v}-\boldsymbol{c}_{2}\right\rangle \geq\left\langle\boldsymbol{f} ; \boldsymbol{v}-\boldsymbol{c}_{2}\right\rangle & \forall \boldsymbol{v} \in \mathcal{K}
\end{array}
$$

Since $\boldsymbol{c}_{1}, \boldsymbol{c}_{2} \in \mathcal{K}$, choose $\boldsymbol{v}=\boldsymbol{c}_{2}$ in (4.13) and $\boldsymbol{v}=\boldsymbol{c}_{1}$ in (4.14). This results in

$$
\begin{aligned}
& \left\langle\boldsymbol{K} \boldsymbol{c}_{1} ; \boldsymbol{c}_{2}-\boldsymbol{c}_{1}\right\rangle \geq\left\langle\boldsymbol{f} ; \boldsymbol{c}_{2}-\boldsymbol{c}_{1}\right\rangle \\
& \left\langle\boldsymbol{K} \boldsymbol{c}_{2} ; \boldsymbol{c}_{1}-\boldsymbol{c}_{2}\right\rangle \geq\left\langle\boldsymbol{f} ; \boldsymbol{c}_{1}-\boldsymbol{c}_{2}\right\rangle
\end{aligned}
$$

Summing the above two inequalities and invoking the linearity in the second slot, we obtain

$$
\left\langle\boldsymbol{K}\left(\boldsymbol{c}_{1}-\boldsymbol{c}_{2}\right) ; \boldsymbol{c}_{1}-\boldsymbol{c}_{2}\right\rangle \leq \mathbf{0}
$$

which further implies that

$$
\left\langle\operatorname{sym}[\boldsymbol{K}]\left(\boldsymbol{c}_{1}-\boldsymbol{c}_{2}\right) ; \boldsymbol{c}_{1}-\boldsymbol{c}_{2}\right\rangle \leq \mathbf{0}
$$

On the other hand, the positive-definiteness of $\operatorname{sym}[\boldsymbol{K}]$ and our assumption $\boldsymbol{c}_{1}-\boldsymbol{c}_{2} \neq \mathbf{0}$ imply that

$$
\left\langle\operatorname{sym}[\boldsymbol{K}]\left(\boldsymbol{c}_{1}-\boldsymbol{c}_{2}\right) ; \boldsymbol{c}_{1}-\boldsymbol{c}_{2}\right\rangle>\mathbf{0}
$$

which contradicts the inequality given by equation (4.18). Hence, $\boldsymbol{c}_{1}=\boldsymbol{c}_{2}$.

Using the aforementioned general existence and uniqueness results for VIs, we now establish the existence and uniqueness of solutions under the proposed framework in the discrete setting.

TheOREM 6. (Well-posedness of the proposed framework) Unique solutions exist for the VIs from the GAL, SUPG and DG WFs under lower bounds (which arise from the non-negative constraint) and under both lower and upper bounds (which arise from maximum principles).

Proof. First, it should be noted that the symmetric part of the coefficient matrices under the GAL and DG formulations are positive-definite. On the other hand, the stabilization term under the SUPG formulation does not guarantee that the symmetric part of the coefficient matrix to be positive-definite. It should also be noted that the stabilization term in equation $(3.10)$ is $\mathcal{O}(h)$, where $h$ is the characteristic mesh size. This means that there exists a critical mesh size, $h_{\text {crit }}$, such that if $h<h_{\text {crit }}$ then the contribution from the residual terms to the coefficient matrix will be small, and the resulting symmetric part of the coefficient matrix will be positive-definite.

(Existence.) If both the lower and upper bounds are present, the feasible region will be compact. For this case of bound constraints, Theorem 2 establishes the existence of solutions for the VIs arising from all the three WFs (i.e., GAL, SUPG and DG). If only the lower bounds are present, Theorem 3 will ensure the existence of solutions for VIs arising from the GAL and DG formulations, and Theorem 4 will ensure the existence of solutions for the VIs arising from the SUPG formulation on a general mesh. Of course, if the mesh is adequately refined (i.e., $h<h_{\text {crit }}$ ) then Theorem 3 can also ensure the existence of a solution for the VIs arising under the SUPG formulation.

(Uniqueness.) Theorem 5 provides the uniqueness of solution for the VIs arising from the GAL and DG formulations. As discussed above, upon an adequate mesh refinement, $\operatorname{sym}[\boldsymbol{K}]$ will be positive-definite under the SUPG formulation. On those meshes, Theorem 5 will provide the uniqueness of solutions for the VIs arising from the SUPG formulation. 
4.2. Computer implementation details. In this paper, the proposed QP and VI-based formulations for the GAL, SUPG, and DG formulations are implemented through the Firedrake project (see Appendix A for further details), but one can employ any other finite element library. The primary advantage of the Firedrake project is that it provides easy access to parallel solvers, specifically the PETSc and TAO libraries [Balay et al., 2016, 1997; Munson et al., 2014] which are built on top of Message Passing Interface (MPI). Appropriate iterative solvers and preconditioners are needed for large-scale problems, and the PETSc library provides the necessary data structures. The Conjugate Gradient (CG) method is used for symmetric problems like the diffusion equation whereas the Generalized Minimal Residual (GMRES) method is used for the non-symmetric advectiondiffusion equation. HYPRE's algebraic multi-grid package [Law] is used as the preconditioner.

4.2.1. Solvers. The main ingredient of the proposed computational framework is to solve finitedimensional VIs. There are several solvers available for solving these type of inequalities in a large-scale parallel environment. However, the performance of these solvers is problem-specific. It is, therefore, necessary to identify the best performing VI solver for our case, which is primarily to enforcing maximum principles and the non-negative constraint. To this end, we consider the following VI and QP solvers available through the PETSc and TAO libraries:

(i) Semi-smooth (VI - SS): TAO's implementation of the semi-smooth algorithm [Luca et al., 1996; Munson et al., 2001] reformulates the MCP as a non-smooth system of equations using the Fischer-Burmeister function [Fischer, 1992]. This function, $\phi: \mathbb{R}^{2} \rightarrow \mathbb{R}$, is defined as

$$
\begin{aligned}
& \phi(a, b):=\sqrt{a^{2}+b^{2}}-a-b \\
& \phi(a, b)=0 \quad \Leftrightarrow \quad a \geq 0, b \geq 0, a b=0
\end{aligned}
$$

The reformulation of the MCP is handled component-wise, and the system of equations $\Phi(\boldsymbol{c})=0$ where $\Phi: \mathbb{R}^{\text {ndofs }} \rightarrow \mathbb{R}^{\text {ndofs }}$ is expressed as:

$$
\Phi_{i}(\boldsymbol{c}):= \begin{cases}\phi\left(c_{i}-c_{\min }, h_{i}(\boldsymbol{c})\right) & \text { if }-\infty<c_{\min }<c_{\max }=\infty \\ \phi\left(c_{\max }-c_{i},-h_{i}(\boldsymbol{c})\right) & \text { if }-\infty=c_{\min }<c_{\max }<\infty \\ \phi\left(c_{i}-c_{\min }, \phi\left(c_{\max }-c_{i},-h_{i}(\boldsymbol{c})\right)\right) & \text { if }-\infty<c_{\min }<c_{\max }<\infty \\ -h_{i}(\boldsymbol{c}) & \text { if }-\infty=c_{\min }<c_{\max }=\infty \\ c_{\min }-c_{i} & \text { if }-\infty<c_{\min }=c_{\max }<\infty\end{cases}
$$

It should be noted that $\Phi(\boldsymbol{c})$ is not differentiable everywhere but it still satisfies a semismoothness property [Mifflin, 1977; Qi, 1993; Qi and Sun, 1993]. The above system of equations is used to compute a descent direction, and the solver finishes as soon as the natural merit function $\Psi(\boldsymbol{c}):=\frac{1}{2}\|\Phi(\boldsymbol{c})\|_{2}^{2}$ meets some level of tolerance. We also employ TAO's feasible line-search algorithm which ensures that the solution is within the bounds by using a projected Armijo line search [Armijo, 1966].

(ii) Reduced-space active-set (VI - RS): The reduced-space active-set method selects an activeset and solves a reduced linear system of equations to calculate a direction of the gradient descent. The active and inactive sets are, respectively, defined as:

$$
\begin{array}{l|l}
\mathcal{A}(\boldsymbol{c}):=\{i \in\{1, \ldots, n d o f s\} & \left.c_{i}=0 \text { and } h_{i}(\boldsymbol{c})>0\right\} \\
\mathcal{I}(\boldsymbol{c}):=\{i \in\{1, \ldots, n d o f s\} & \left.c_{i}>0 \text { or } h_{i}(\boldsymbol{c}) \leq 0\right\}
\end{array}
$$

The active set $\mathcal{A}(\boldsymbol{c})$ represents regions where the lower bound is active thus the function value can be ignored, and the inactive set $\mathcal{I}(\boldsymbol{c})$ contains everything else. The descent direction of 
the active set is set to zero whereas the descent direction of the inactive set is approximated, and the solution is updated using a projected line search. Unlike the standard clipping procedure, this method is still variationally consistent because only the gradient descent is clipped and not the actual solution itself. As far as we know, there is little documentation on the theoretical and mathematical convergence properties for this particular algorithm, but the computational results from [Benson and Munson, 2006] demonstrate that this solver is robust and can handle a wide range of applications. For further implementation details of these two VI solvers, we defer all interested readers to [Benson and Munson, 2006; Munson et al., 2014] and the references within.

(iii) Trust region Newton (QP - TRON): Unlike the previous two solvers, the trust region Newton method [Lin and Moré, 1999] is an active-set solver designed for large-scale minimization problems. It uses the gradient projection to generate a Cauchy step and the preconditioned $\mathrm{CG}$ with an incomplete Cholesky factorization to generate a descent direction. Each iteration of the TRON algorithm solves a reduced linear system containing variables that lie between the lower and upper bounds. The algorithm then applies a trust region to the conjugate gradients to ensure convergence. The algorithmic scalability and hardware performance of this solver has been thoroughly documented in [Chang et al., 2017], so the computational results arising from QP - TRON serves primarily as a benchmark for comparison with the VI solvers.

We acknowledge that there may be several other QP and VI solvers which are not covered in this paper. Nonetheless, the computational framework that we propose is algorithm-independent and platform-agnostic, so one is free to either employ different solvers or modify the above implementations of the QP and VI algorithms to cater to specific needs and applications.

4.2.2. An outline of the algorithm. The performance of non-linear and optimization-based solvers depends on accurate initial guesses. To this end, we propose the following steps for the overall implementation of the proposed computational framework:

Step 1: Assemble $\boldsymbol{K}$ and $\boldsymbol{f}$

Step 2: Solve for $\boldsymbol{c}_{0}$.

Step 3: Clip $\boldsymbol{c}_{0}$ and obtain $\boldsymbol{c}_{\mathrm{CLIP}}$. Formally:

$$
\boldsymbol{c}_{\mathrm{CLIP}}=\arg \min \frac{1}{2}\left\|\boldsymbol{c}-\boldsymbol{c}_{0}\right\|^{2} \quad \text { subject to: } c_{\min } \mathbf{1} \preceq \boldsymbol{c} \preceq c_{\max } \mathbf{1}
$$

Step 4: Solve the bounded constraint problem under the QP or VI framework with $\boldsymbol{c}_{\mathrm{CLIP}}$ as the initial guess.

It should be emphasized that one need not solve equation (4.23) to implement clipping procedure. Instead, one trims nodal values to meet the desired bounds. Since the governing equations are linear, $\boldsymbol{K}$ and $\boldsymbol{f}$ only need to be assembled once and are reused for the various QP and VI evaluation routines for Step 4. Python implementations of the VI - SS, VI - RS, and QP - TRON solvers leveraging petsc4py [Dalcin et al., 2011] capabilities are shown in Listings 5, 6, and 7 respectively of Appendix A. For the steady-state 3D benchmarks in the next section, the KSP relative tolerance is set to $10^{-7}$ for the solver in Step 2, whereas the KSP relative tolerance for approximating the gradient descents in Step 4 is set to $10^{-3}$. It was shown in [Chang et al., 2017] that relaxing the tolerance requires more non-linear iterations but lessens the overall solve time. Relaxing the tolerance also lessens the arithmetic intensity where the performance is governed by the memory bandwidth thus making it less likely to achieve good speedup on a shared compute node. In other 


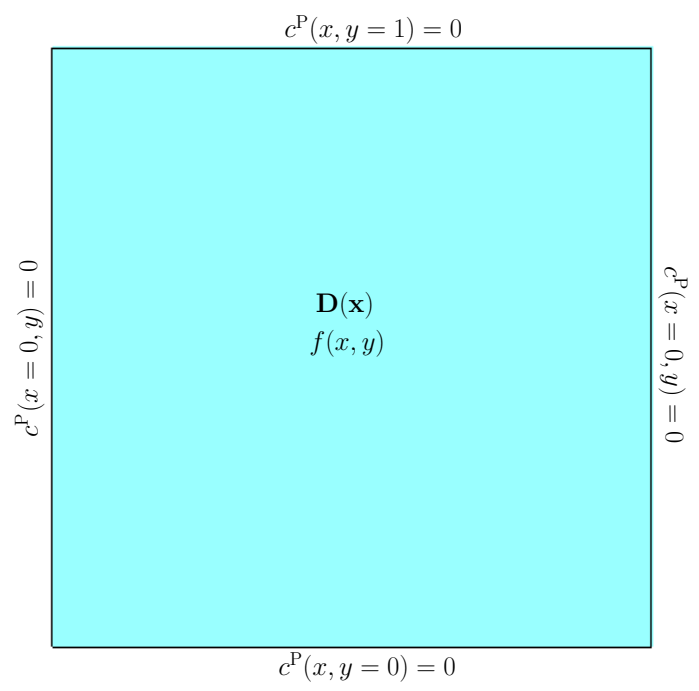

(A) Diffusion problem

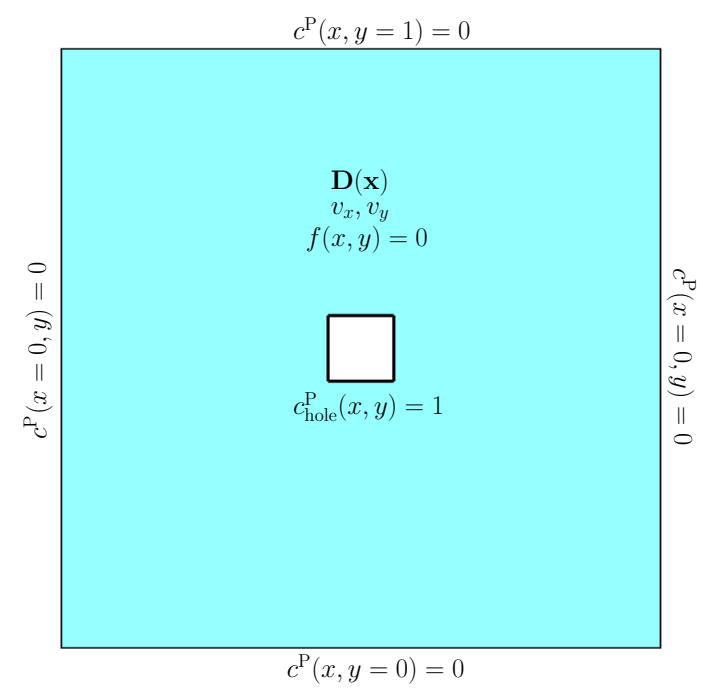

(B) Advection-diffusion problem

FiguRE 4. 2D benchmarks: Pictorial description of the boundary value problems for the diffusion and advection-diffusion examples. The finite element mesh for the diffusion problem contains 40,000 quadrilateral elements and 40,401 nodes. The mesh for the advection-diffusion problem is unstructured containing 96,430 triangular elements and 48,663 nodes.

words, the parallel efficiency of the QP and VI solvers are likely to be worse than solving the WF with standard KSP convergence tolerances. The absolute convergence tolerances for the QP and VI solvers are set to $10^{-8}$ although it should be mentioned that the optimal values depends on the application at hand. All large-scale computations are conducted on Intel Xeon E5-2680v2 processors where each MPI process is restricted to a single core.

\section{NUMERICAL RESULTS FOR STEADY-STATE RESPONSE}

5.1. 2D benchmarks. We now examine $2 \mathrm{D}$ problems in order to demonstrate the effectiveness of the proposed computational algorithms for ensuring discrete maximum principles and the nonnegative constraint. Only the GAL and SUPG formulations are employed in this numerical study. First, let us consider the pure diffusion equation on a bi-unit square: $\Omega:=(0,1) \times(0,1)$ as shown in Figure 4a. The following heterogeneous and anisotropic diffusivity tensor similar to the one considered in [Potier, 2005] is used:

$$
\mathbf{D}(\mathbf{x})=\left(\begin{array}{cc}
y^{2}+\epsilon x^{2} & -(1-\epsilon) x y \\
-(1-\epsilon) x y & x^{2}+\epsilon y^{2}
\end{array}\right)
$$

where $\epsilon=10^{-4}$. The forcing function is defined as $f(x, y)=1$ if $(x, y) \in\left[\frac{3}{8}, \frac{5}{8}\right] \times\left[\frac{3}{5}, \frac{5}{8}\right]$ and zero elsewhere. Homogeneous boundary conditions are applied on all four sides of the domain. Numerical solutions under the GAL, VI - SS, VI - RS, and QP - TRON methods with uniform quadrilateral elements of $h$-size $=1 / 200$ are shown in Figure 5. All three non-negative solvers 


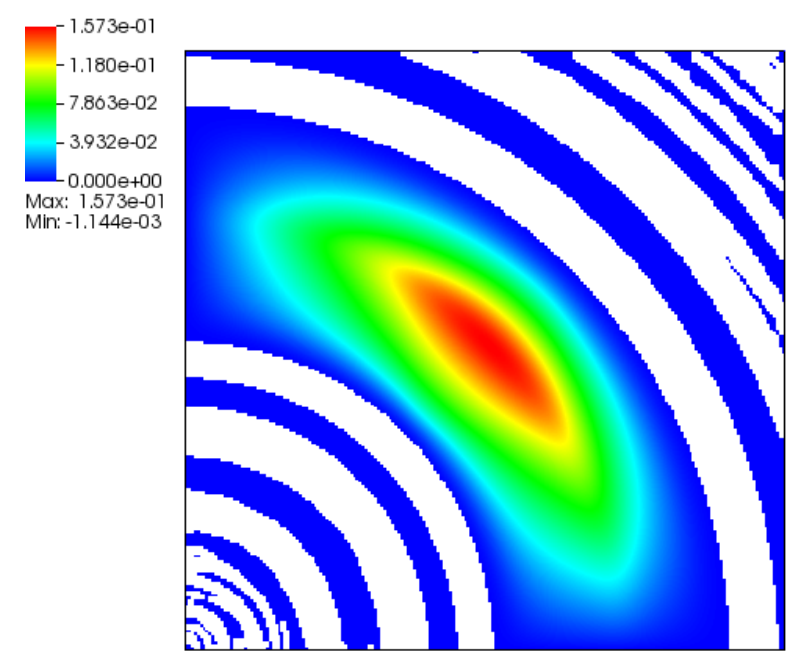

(A) $\boldsymbol{c}_{\mathrm{GAL}}$

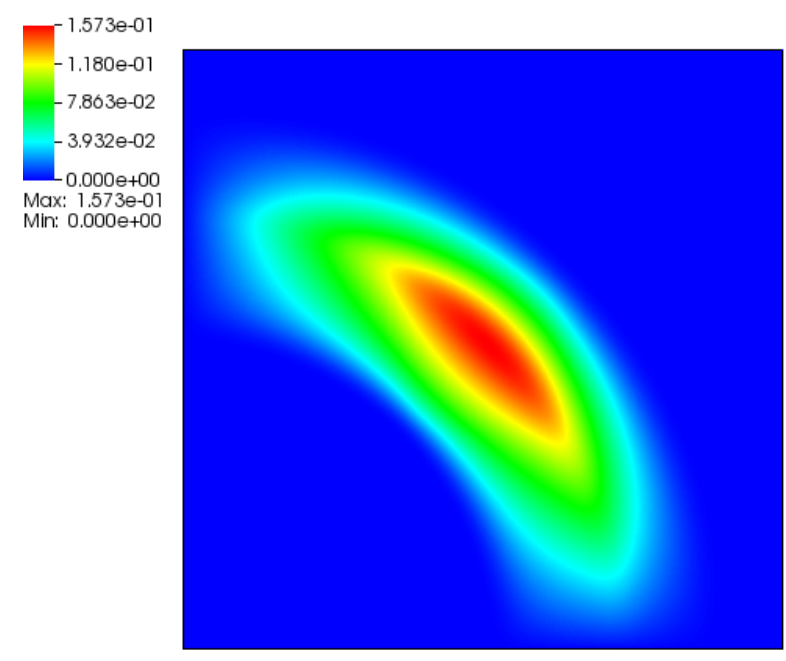

(C) $\boldsymbol{c}_{\mathrm{SS}}$

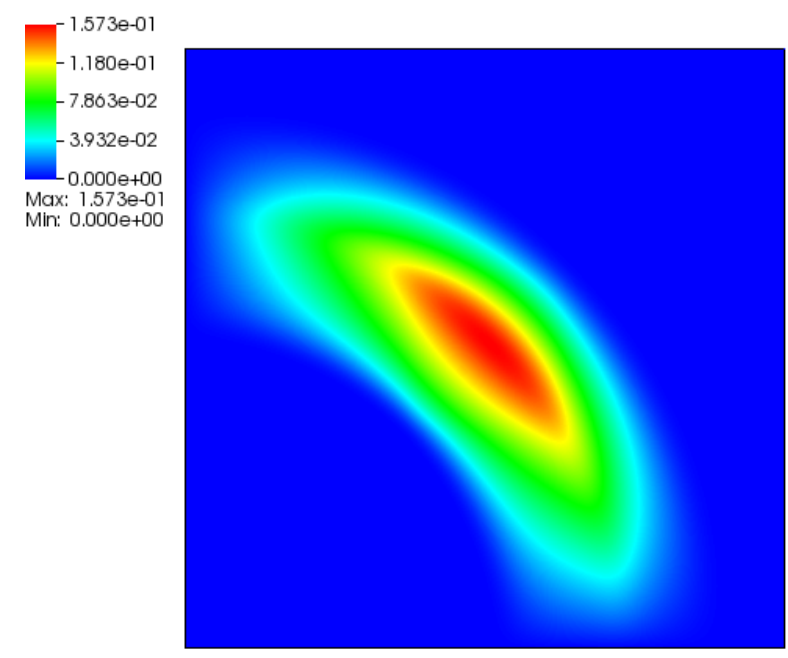

(B) $\boldsymbol{c}_{\mathrm{TRON}}$

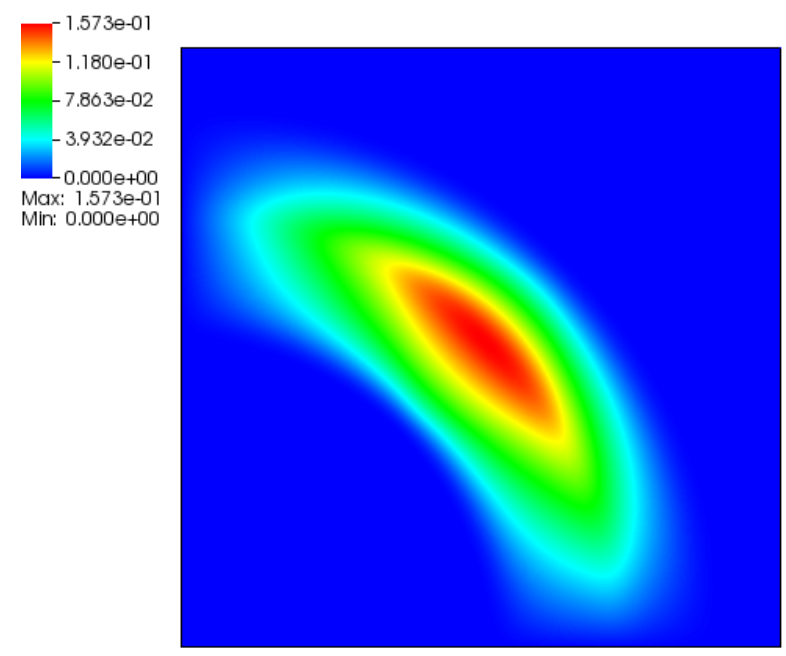

(D) $\boldsymbol{c}_{\mathrm{RS}}$

Figure 5. 2D diffusion: concentrations under the Galerkin ( $\left.\boldsymbol{c}_{\mathrm{GAL}}\right)$, TRON $\left(\boldsymbol{c}_{\mathrm{TRON}}\right)$, semi-smooth $\left(\boldsymbol{c}_{\mathrm{SS}}\right)$, and reduced-space active-set $\left(\boldsymbol{c}_{\mathrm{RS}}\right)$ methods where the white regions represent negative concentrations.

successfully eliminate negative concentrations, and the absolute difference plots in Figure 6 show that their results are quite different than from the one arising from the standard clipping procedure. Moreover, the absolute differences between the various QP and VI solvers, as seen from Figure 7, are extremely small and suggest that the QP and VI solvers have similar numerical accuracy. 


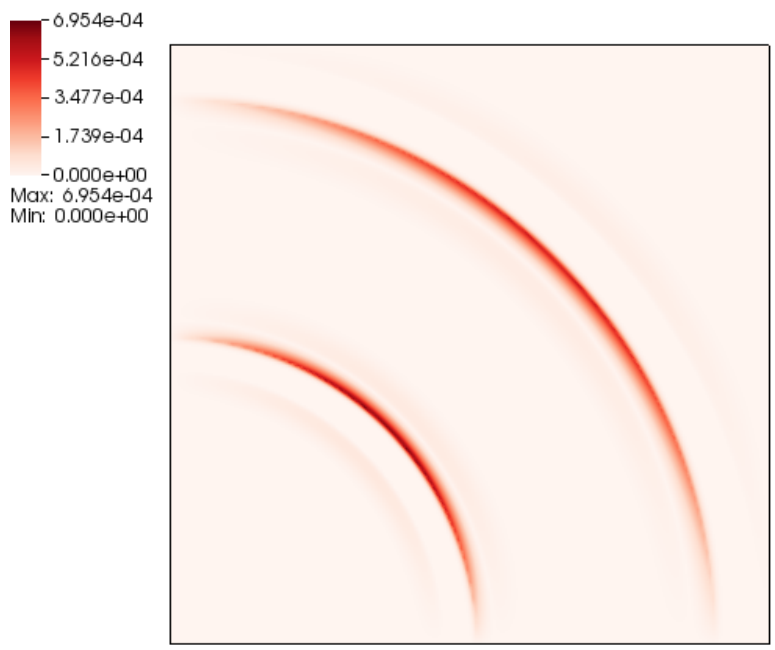

(A) $\left\|\boldsymbol{c}_{\mathrm{CLIP}}-\boldsymbol{c}_{\mathrm{TRON}}\right\|$
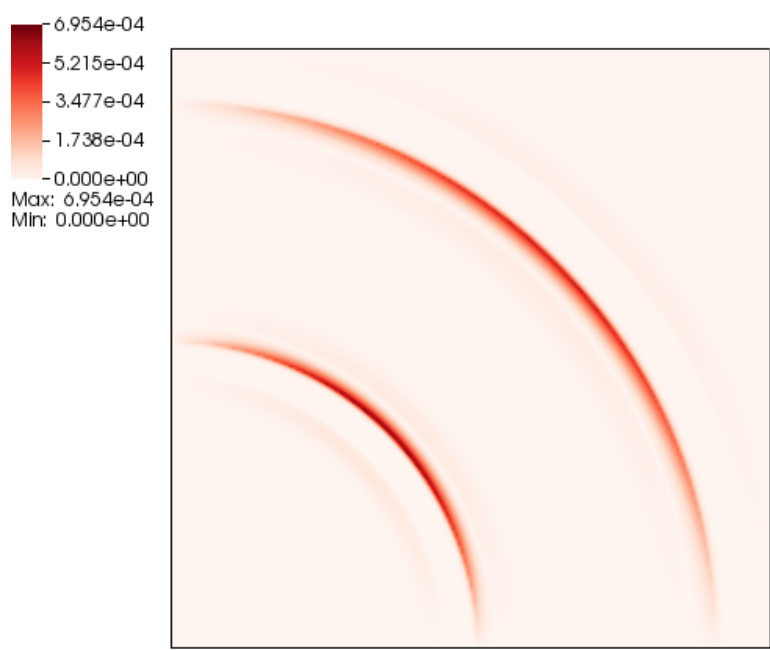

(в) $\left\|\boldsymbol{c}_{\mathrm{CLIP}}-\boldsymbol{c}_{\mathrm{SS}}\right\|$

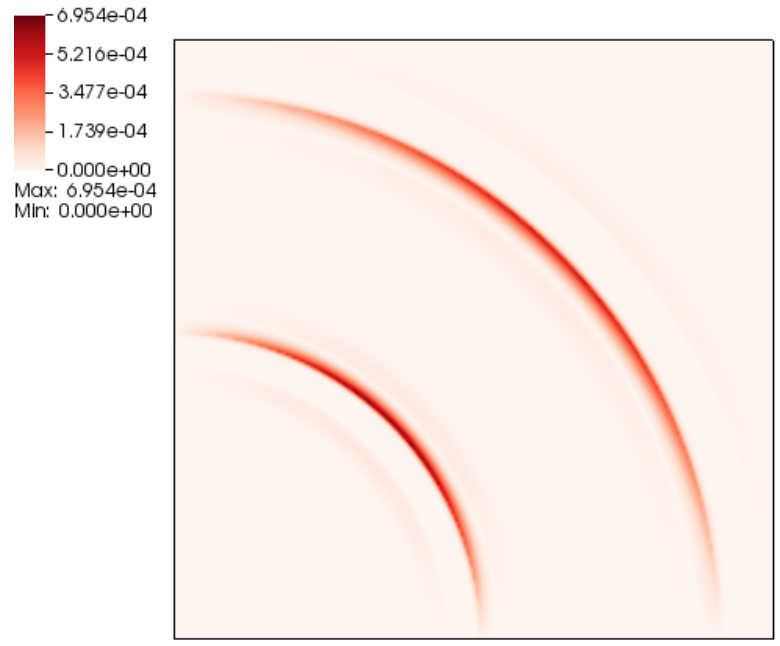

(C) $\left\|\boldsymbol{c}_{\mathrm{CLIP}}-\boldsymbol{c}_{\mathrm{RS}}\right\|$

FiguRE 6. 2D diffusion: absolute difference in concentrations between the clipped solution and the non-negative solution.

Next we consider the advection-diffusion problem under the SUPG formulation where only VI SS and VI - RS methods are applicable for enforcing the maximum principle and the non-negative constraint. Consider a bi-unit square: $\Omega:=(0,1) \times(0,1)$ with a square hole of dimension $\left[\frac{4}{9}, \frac{5}{9}\right] \times$ $\left[\frac{4}{9}, \frac{5}{9}\right]$ as shown in Figure 4b. The mesh is discretized into 96,430 unstructured triangular elements and 48,663 vertices. Homogeneous boundary conditions are applied on the outside boundary, and 


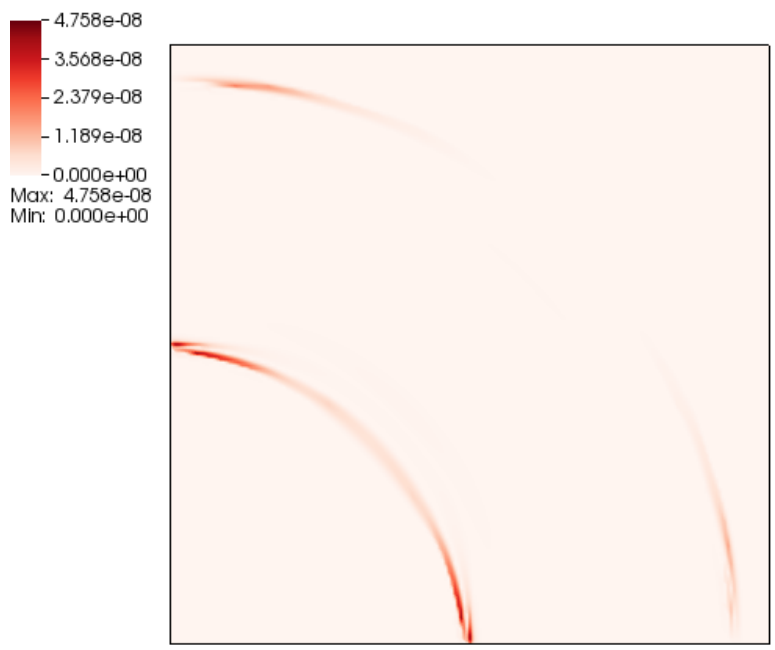

(A) $\left\|\boldsymbol{c}_{\mathrm{TRON}}-\boldsymbol{c}_{\mathrm{SS}}\right\|$

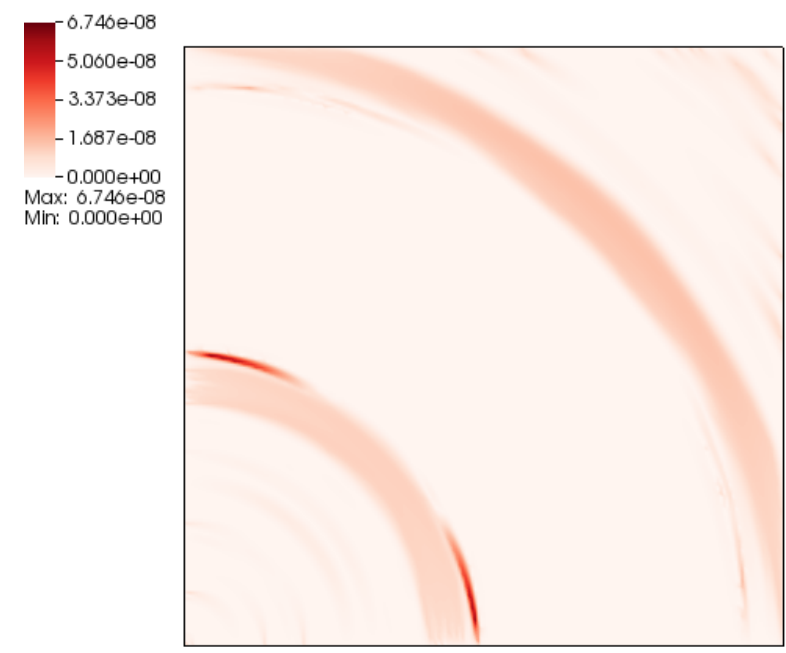

(B) $\left\|c_{\mathrm{TRON}}-\boldsymbol{c}_{\mathrm{RS}}\right\|$

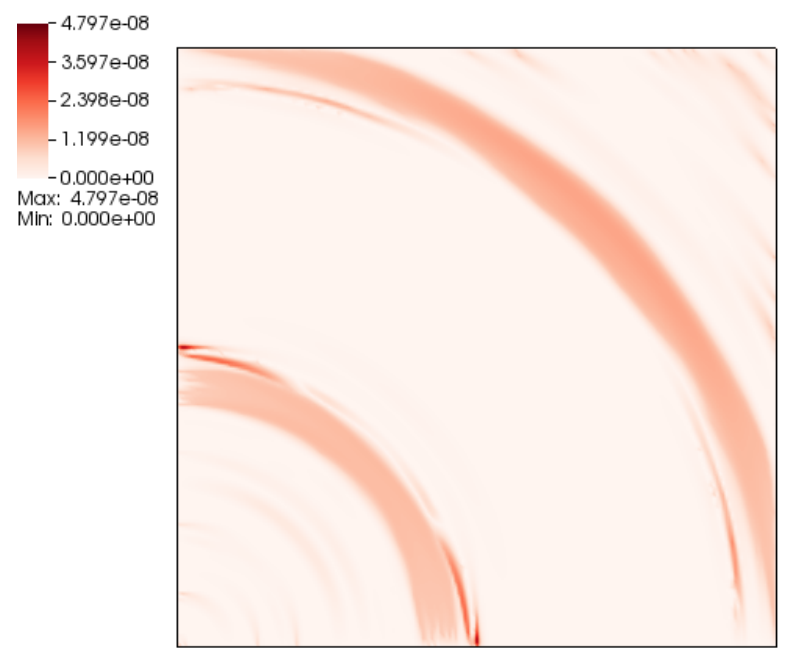

(C) $\left\|c_{\mathrm{SS}}-\boldsymbol{c}_{\mathrm{RS}}\right\|$

FiguRE 7. 2D diffusion: absolute difference in concentrations between the various non-negative methodologies

a Dirichlet boundary value $c^{\mathrm{p}}(\mathbf{x})=1$ is applied on the interior boundary $\Gamma^{\text {hole }}$. The velocity vector field $\mathbf{v}(\mathbf{x})$ is characterized by the following:

$$
\begin{aligned}
& v_{x}=\cos \left(2 \pi y^{2}\right) \\
& v_{y}=\sin (2 \pi x)+\cos \left(2 \pi x^{2}\right)
\end{aligned}
$$




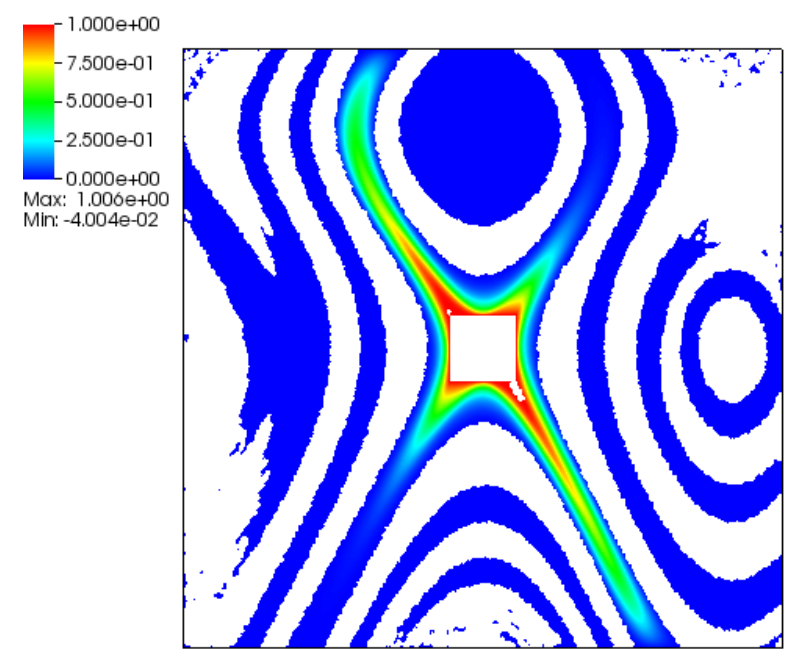

(A) $\boldsymbol{c}_{\mathrm{SUPG}}$

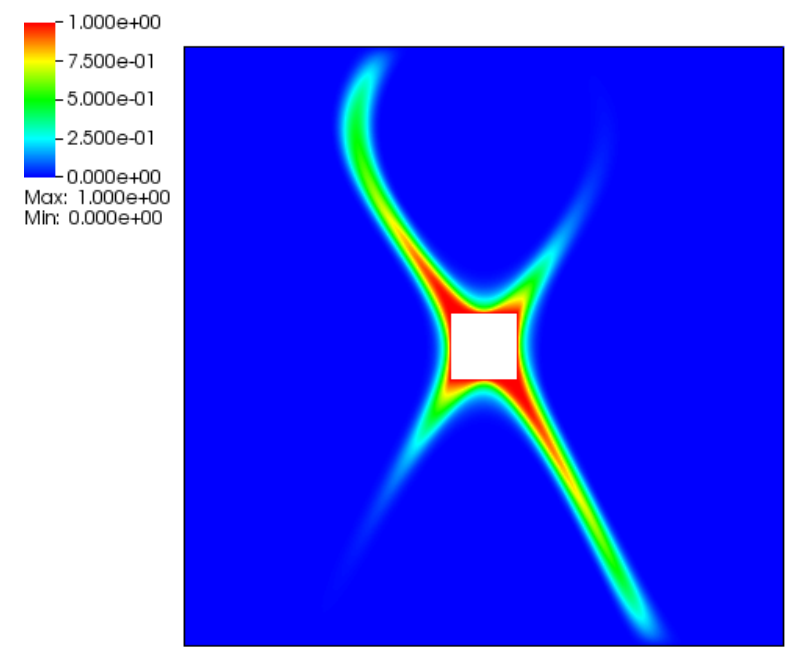

(B) $\boldsymbol{c}_{\mathrm{SS}}$

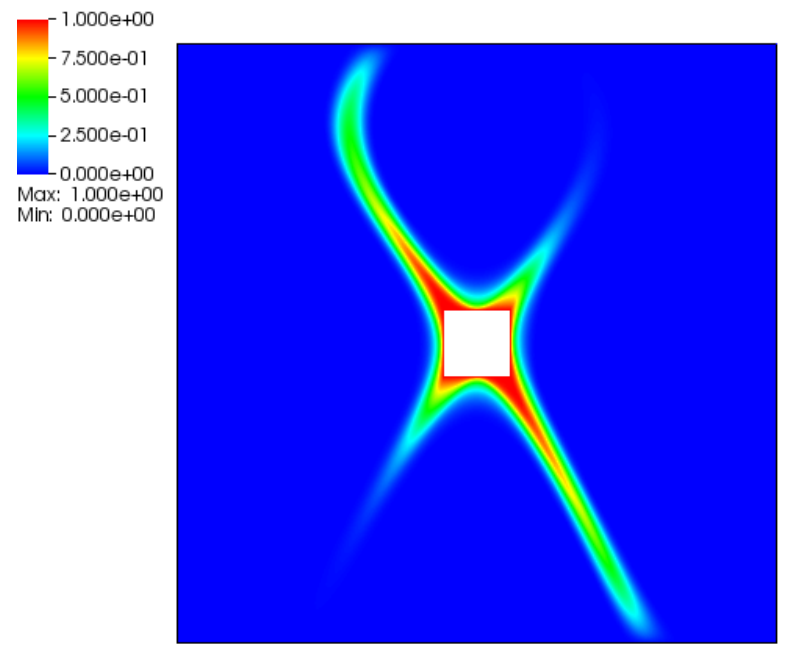

(C) $\boldsymbol{c}_{\mathrm{RS}}$

FigURE 8. 2D advection-diffusion: concentrations under the SUPG ( $\left.\boldsymbol{c}_{\mathrm{SUPG}}\right)$, semismooth $\left(\boldsymbol{c}_{\mathrm{SS}}\right)$, and reduced-space active-set $\left(\boldsymbol{c}_{\mathrm{RS}}\right)$ methods where the white regions represent negative concentrations.

and the diffusivity tensor $\mathbf{D}(\mathbf{x})$ for this problem is the dispersion tensor:

$$
\mathbf{D}(\mathbf{x})=\left(\alpha_{T}\|\mathbf{v}\|+D_{M}\right) \mathbf{I}+\left(\alpha_{L}-\alpha_{T}\right) \frac{\mathbf{v} \otimes \mathbf{v}}{\|\mathbf{v}\|}
$$




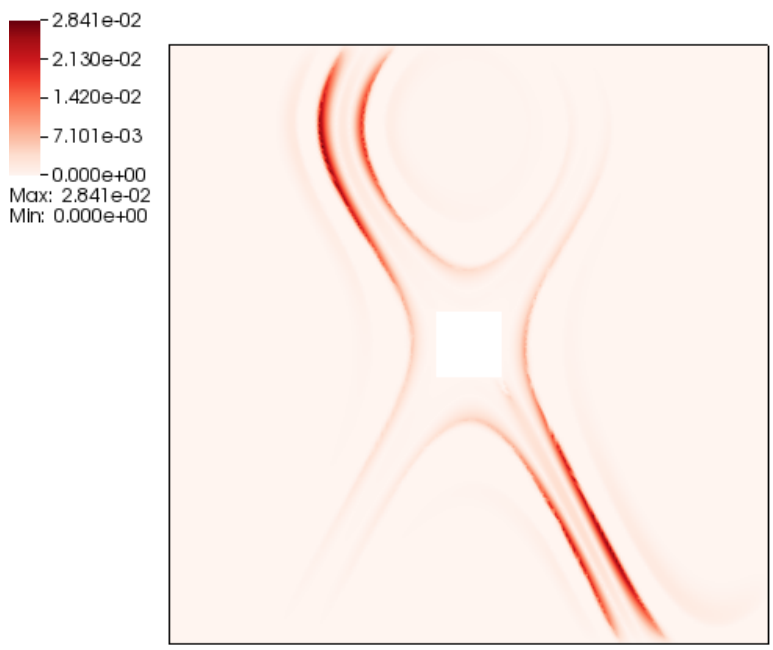

(A) $\left\|c_{\mathrm{CLIP}}-\boldsymbol{c}_{\mathrm{SS}}\right\|$

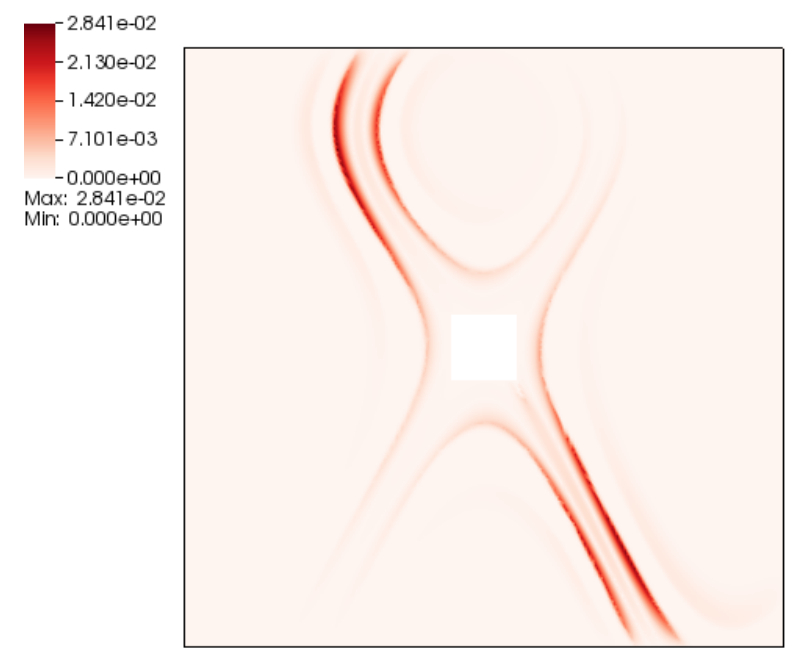

(в) $\left\|\boldsymbol{c}_{\mathrm{CLIP}}-\boldsymbol{c}_{\mathrm{RS}}\right\|$

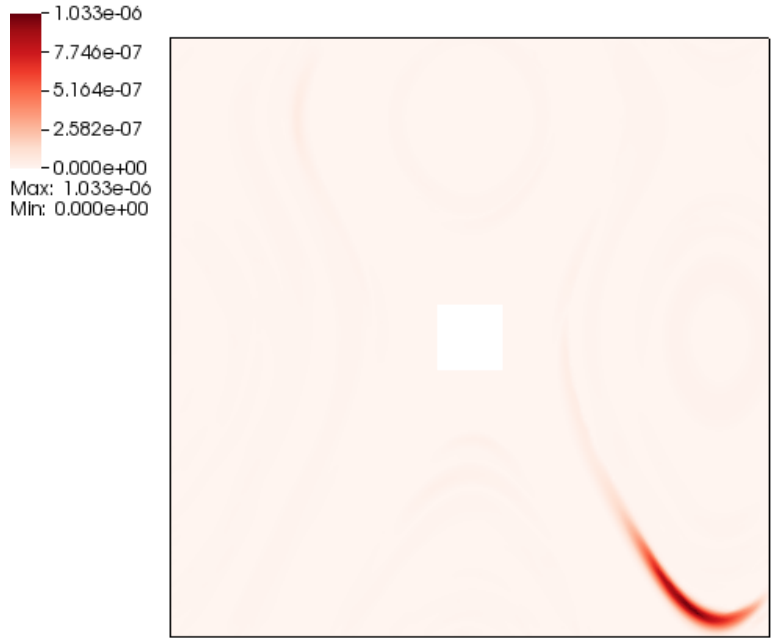

(C) $\left\|c_{\mathrm{SS}}-\boldsymbol{c}_{\mathrm{RS}}\right\|$

FiguRE 9. 2D advection-diffusion: absolute difference in concentrations between the clipped and non-negative solutions.

where $\alpha_{L}=10^{-1}, \alpha_{T}=10^{-5}$, and $D_{M}=10^{-9}$ denote the longitudinal dispersivity, transverse dispersivity and molecular diffusivity, respectively. Figure 8 depicts the numerical solutions under the SUPG, VI - SS, and VI - RS formulations. We see that the SUPG formulation results in negative concentrations as well as concentrations greater than the maximum prescribed boundary condition whereas the two VI solvers successfully correct these concentrations. The absolute difference plots, 

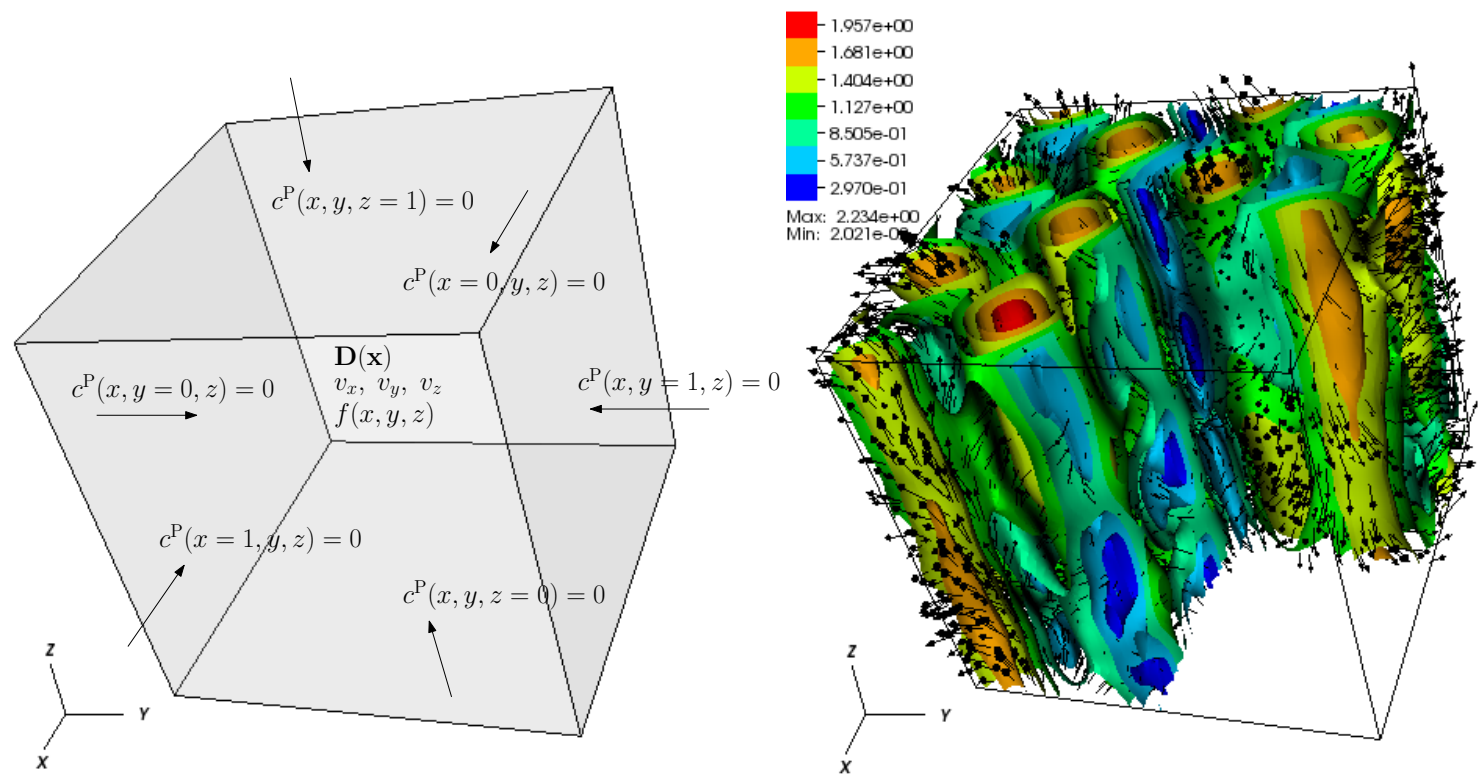

FiguRE 10. 3D benchmarks: Left figure contains a pictorial description of the boundary value problem. Right figure contains the corresponding velocity contour and vector field for the ABC flow (right) for the steady-state diffusion and advectiondiffusion problems.

as seen in Figure 9, indicate that the VI are also similar to one another and differ from the clipping procedure. These $2 \mathrm{D}$ benchmarks suggest that the $\mathrm{QP}$ and VI solvers are accurate alternatives to the clipping procedure for satisfying the discrete maximum principle and the non-negative constraint. Listings 1 and 2 contain the Firedrake project files for solving the GAL and SUPG formulations, respectively.

5.2. 3D benchmark. We now consider a 3D problem designed to capture two particular aspects that may arise in large-scale applications: 1) chaotic advection, which is pervasive in many porous media applications [Lester et al., 2013], and 2) random point sources, which in subsurface remediation problems are the sites where potential contaminant leaks occur. Predictive modeling involving such important aspects require numerical methodologies that are not only accurate but also fast and scalable in a parallel environment. Herein, our goal is to study the computational performance of the various QP and VI solvers under the GAL, SUPG, and DG formulations.

Consider a unit cube domain as shown in Figure 10 with chaotic advection flow characterized by the Arnold-Beltrami-Childress (ABC) flow [Zhao et al., 1993; Dombre et al., 1986]:

$$
\begin{aligned}
& v_{x}=0.3 \sin (2 \pi z)+\cos (3 \pi y) \\
& v_{y}=0.65 \sin (2 \pi x)+0.3 \cos (5 \pi z) \\
& v_{z}=\sin (4 \pi y)+0.65 \cos (6 \pi y)
\end{aligned}
$$

For this problem, we shall also let $\mathbf{D}(\mathbf{x})$ denote the dispersion tensor as shown in equation (5.3) where $\alpha_{L}=10^{-1}, \alpha_{T}=10^{-5}$, and $D_{M}=10^{-9}$. All six faces of the cube have homogeneous 


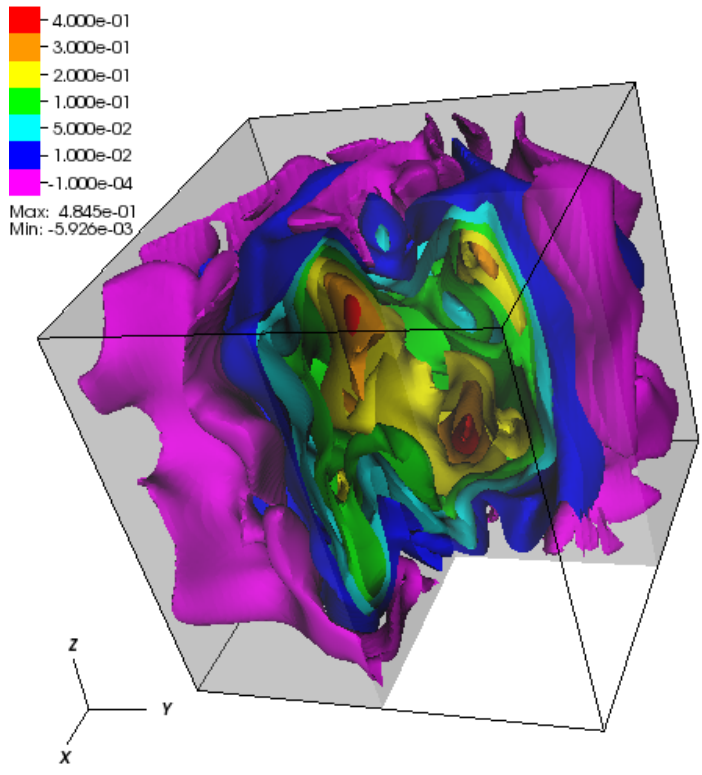

(A) GAL

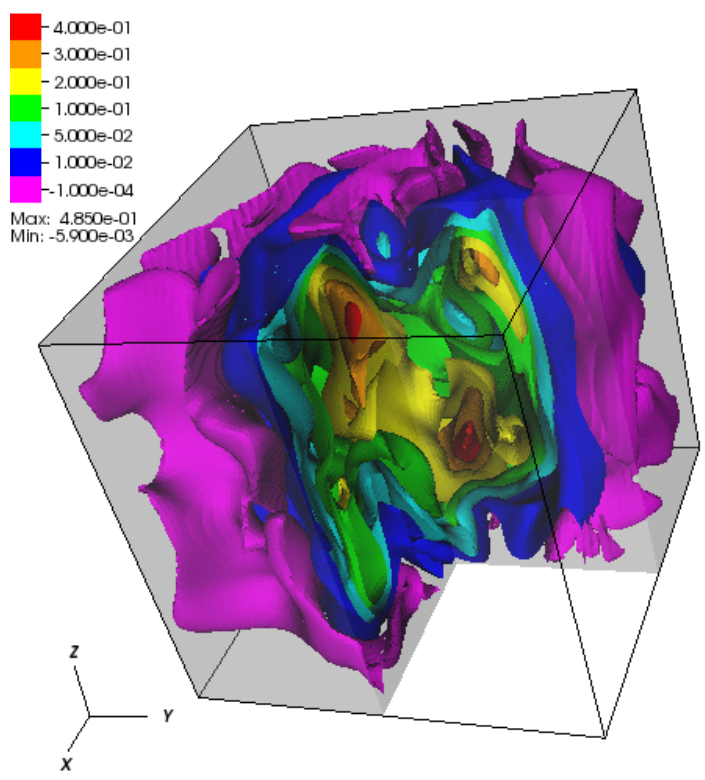

(c) DG

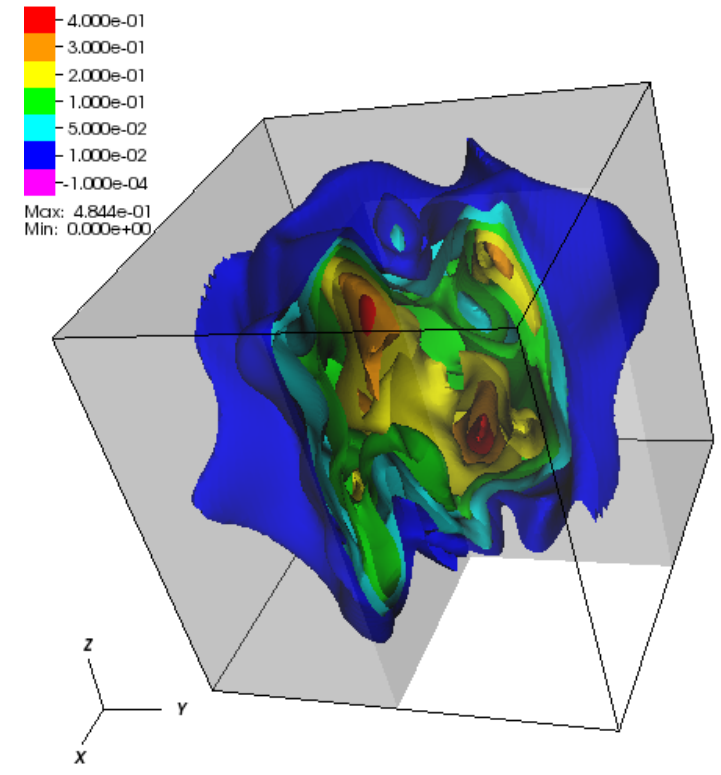

(в) GAL with VI - SS

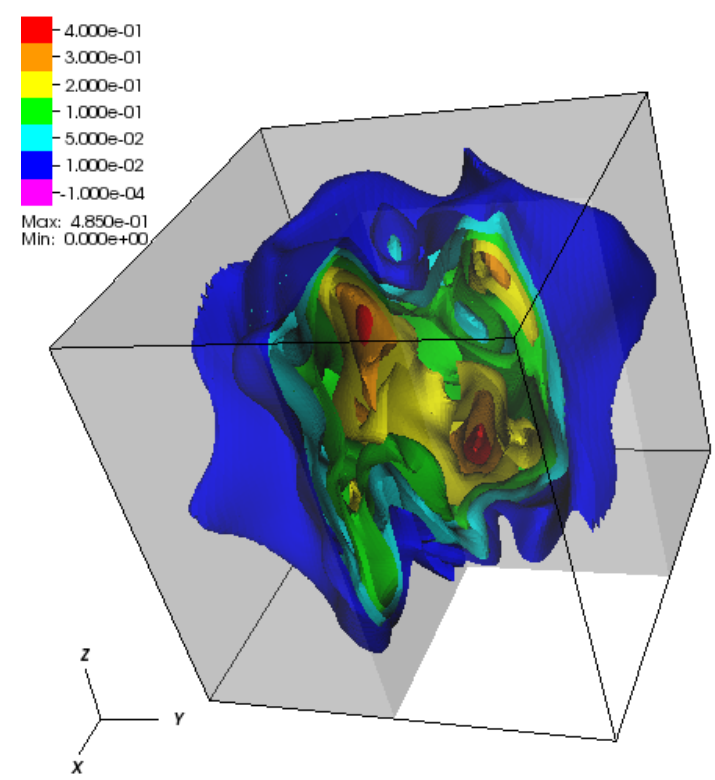

(D) DG with VI - SS

Figure 11. 3D diffusion: 3D contours of the concentrations for the GAL and DG formulations with and without and VI - SS for $h$-size $=1 / 80$, where the purple contours represent regions with negative concentrations (see online version for color figures.)

boundary conditions, and the following forcing function consisting of 8 randomly located point 
TABLE 1. 3D diffusion: minimum and maximum concentrations for various level of mesh refinement under the GAL formulation.

\begin{tabular}{lccc}
\hline$h$-size & Min. concentration & Max. concentration & \% degrees-of-freedom violated \\
\hline $1 / 10$ & -0.0224497 & 0.368322 & $280 / 1,331 \rightarrow 21.0 \%$ \\
$1 / 20$ & -0.0071611 & 0.339679 & $2,462 / 9,261 \rightarrow 26.6 \%$ \\
$1 / 30$ & -0.0083804 & 0.481598 & $8,449 / 29,791 \rightarrow 28.4 \%$ \\
$1 / 40$ & -0.0062918 & 0.378390 & $20,195 / 68,921 \rightarrow 29.3 \%$ \\
$1 / 50$ & -0.0067679 & 0.477119 & $39,500 / 132,651 \rightarrow 29.8 \%$ \\
$1 / 60$ & -0.0072030 & 0.518469 & $68,161 / 226,981 \rightarrow 30.0 \%$ \\
$1 / 70$ & -0.0066007 & 0.498127 & $109,554 / 357,911 \rightarrow 30.6 \%$ \\
$1 / 80$ & -0.0059264 & 0.484484 & $160,925 / 531,441 \rightarrow 30.3 \%$ \\
\hline
\end{tabular}

TABLE 2. 3D diffusion: minimum and maximum concentrations for various level of mesh refinement under the DG formulation.

\begin{tabular}{lccc}
\hline$h$-size & Min. concentration & Max. concentration & \% degrees-of-freedom violated \\
\hline $1 / 10$ & -0.0226040 & 0.372831 & $3,704 / 8,000 \rightarrow 46.3 \%$ \\
$1 / 20$ & -0.0071913 & 0.341955 & $27,496 / 64,000 \rightarrow 43.0 \%$ \\
$1 / 30$ & -0.0082811 & 0.483264 & $91,176 / 216,000 \rightarrow 42.2 \%$ \\
$1 / 40$ & -0.0062341 & 0.379389 & $213,000 / 512,000 \rightarrow 41.6 \%$ \\
$1 / 50$ & -0.0067168 & 0.478146 & $410,976 / 1,000,000 \rightarrow 41.1 \%$ \\
$1 / 60$ & -0.0071682 & 0.519338 & $702,504 / 1,728,000 \rightarrow 40.7 \%$ \\
$1 / 70$ & -0.0065727 & 0.498775 & $1,114,856 / 2,744,000 \rightarrow 40.6 \%$ \\
$1 / 80$ & -0.0058998 & 0.485012 & $1,624,496 / 4,096,000 \rightarrow 39.7 \%$ \\
\hline
\end{tabular}

sources is used throughout the domain:

$$
f(x, y, z)= \begin{cases}1 & \text { if }(x, y, z) \in[0.4,0.2,0.1] \times[0.5,0.3,0.2] \\ 1 & \text { if }(x, y, z) \in[0.8,0.4,0.2] \times[0.9,0.5,0.3] \\ 1 & \text { if }(x, y, z) \in[0.5,0.7,0.3] \times[0.6,0.8,0.4] \\ 1 & \text { if }(x, y, z) \in[0.3,0.5,0.2] \times[0.4,0.6,0.3] \\ 1 & \text { if }(x, y, z) \in[0.5,0.2,0.6] \times[0.6,0.3,0.7] \\ 1 & \text { if }(x, y, z) \in[0.6,0.5,0.7] \times[0.7,0.6,0.8] \\ 1 & \text { if }(x, y, z) \in[0.4,0.7,0.8] \times[0.5,0.8,0.9] \\ 1 & \text { if }(x, y, z) \in[0.1,0.4,0.7] \times[0.2,0.5,0.8] \\ 0 & \text { otherwise }\end{cases}
$$

To understand the parallel and algorithmic scalability of the QP and VI solvers, various levels of mesh refinement are considered, ranging from 1,331 to 1,030,301 degrees-of-freedom for the GAL/SUPG formulations and ranging from 8,000 to 4,096,000 degrees-of-freedom for the DG formulations. Up to $16 \mathrm{MPI}$ processes are used to study the weak-scaling and strong-scaling potential of these solvers.

Figure 11 depicts the GAL and DG solutions for the diffusion equation with and without VI - SS. It can be seen from the figures that negative concentrations are present regardless which finite element formulation is used. Tables 1 and 2 indicate that negative concentrations arise for 
TABLE 3. 3D diffusion: wall-clock time and number of solver iterations (KSP, VI, or QP) for various levels of mesh refinement under the GAL formulation.

\begin{tabular}{l|cc|cc|cc|cc}
\hline \multirow{2}{*}{$h$-size } & \multicolumn{2}{|c|}{ GAL } & \multicolumn{2}{c|}{ VI - SS } & \multicolumn{2}{c|}{ VI - RS } & \multicolumn{2}{c}{ QP - TRON } \\
& time (s) & iters & time $(\mathrm{s})$ & iters & time $(\mathrm{s})$ & iters & time $(\mathrm{s})$ & iters \\
\hline $1 / 10$ & 0.003 & 9 & 0.027 & 5 & 0.008 & 2 & 0.007 & 2 \\
$1 / 20$ & 0.036 & 15 & 0.477 & 12 & 0.147 & 5 & 0.135 & 5 \\
$1 / 30$ & 0.165 & 20 & 2.624 & 18 & 0.765 & 7 & 0.650 & 6 \\
$1 / 40$ & 0.525 & 24 & 7.576 & 20 & 2.246 & 8 & 1.758 & 6 \\
$1 / 50$ & 1.293 & 28 & 21.49 & 27 & 5.381 & 9 & 5.330 & 9 \\
$1 / 60$ & 2.556 & 31 & 43.72 & 30 & 12.01 & 11 & 12.20 & 11 \\
$1 / 70$ & 4.747 & 35 & 76.21 & 31 & 18.27 & 10 & 17.81 & 9 \\
$1 / 80$ & 7.962 & 39 & 140.7 & 37 & 36.40 & 13 & 38.06 & 13 \\
\hline
\end{tabular}

TABLE 4. 3D diffusion: wall-clock time and number of solver iterations (KSP, VI, or QP) for various levels of mesh refinement under the DG formulation.

\begin{tabular}{l|cc|cc|cc|cc}
\hline \multirow{2}{*}{$h$-size } & \multicolumn{2}{|c|}{ DG } & \multicolumn{2}{c|}{ VI - SS } & \multicolumn{2}{c|}{ VI - RS } & \multicolumn{2}{c}{ QP - TRON } \\
& time (s) & iters & time (s) & iters & time (s) & iters & time (s) & iters \\
\hline $1 / 10$ & 0.030 & 10 & 0.748 & 12 & 0.221 & 5 & 0.186 & 5 \\
$1 / 20$ & 0.446 & 14 & 1.410 & 20 & 4.528 & 8 & 3.715 & 7 \\
$1 / 30$ & 2.148 & 18 & 73.52 & 27 & 23.85 & 10 & 22.64 & 10 \\
$1 / 40$ & 6.278 & 21 & 251.5 & 34 & 69.33 & 11 & 70.77 & 11 \\
$1 / 50$ & 14.29 & 24 & 623.6 & 39 & 171.7 & 13 & 170.8 & 12 \\
$1 / 60$ & 28.25 & 27 & 1290 & 45 & 360.1 & 15 & 388.6 & 15 \\
$1 / 70$ & 51.95 & 31 & 2560 & 51 & 620.2 & 16 & 639.0 & 15 \\
$1 / 80$ & 85.53 & 34 & 5049 & 54 & 1107 & 19 & 1291 & 17 \\
\hline
\end{tabular}

the GAL and DG formulations, respectively, even as $h$-size is refined. It is interesting to note that the DG formulation not only has more degrees-of-freedom but has more regions with negative concentrations than its GAL counterpart. Using the initial guess solver from Step 2 of the proposed framework in 4.2.2 as a baseline for comparison, Tables 3 and 4 demonstrate how the wall-clock time and number of KSP/VI/QP solver iterations vary with $h$-refinement under a single MPI process. It should be noted that the timings for the QP and VI solvers consider both the assembly of the data structures as well as the actual solver. The heterogeneous nature of the problem causes the number of solvers iterations to increase with problem size, but the iteration counts begin to stabilize as the problem gets bigger. The VI - RS method outperforms VI - SS in both wall-clock time and VI iterations but has similar performance to QP - TRON.

Next we perform weak-scaling studies to investigate how increasing both problem size and number of MPI processes affects the performance of the VI and QP solvers. Each MPI process will handle approximately 100k degrees-of-freedom so the $h$-sizes for the GAL case are $1 / 46,1 / 58$, $1 / 73,1 / 92$, and $1 / 116$ for $1,2,4,8$, and 16 processes respectively whereas the $h$-sizes for the DG case are $1 / 23,1 / 29,1 / 37,1 / 46$, and $1 / 58$ for $1,2,4,8$, and 16 processes respectively. Figure 12 contains the scaling plots as well as the parallel efficiencies in the weak sense under the GAL and DG formulations. Generally speaking, the non-negative methodologies do not scale as well in the weak 


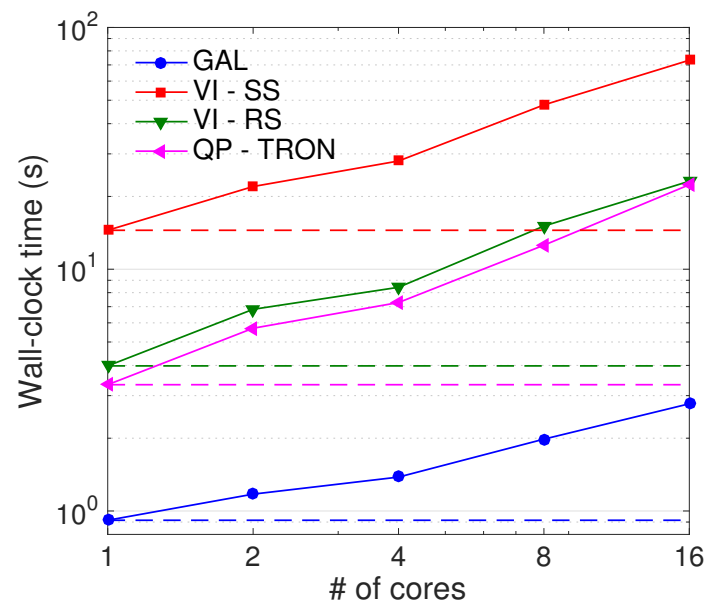

(A) GAL - solve time

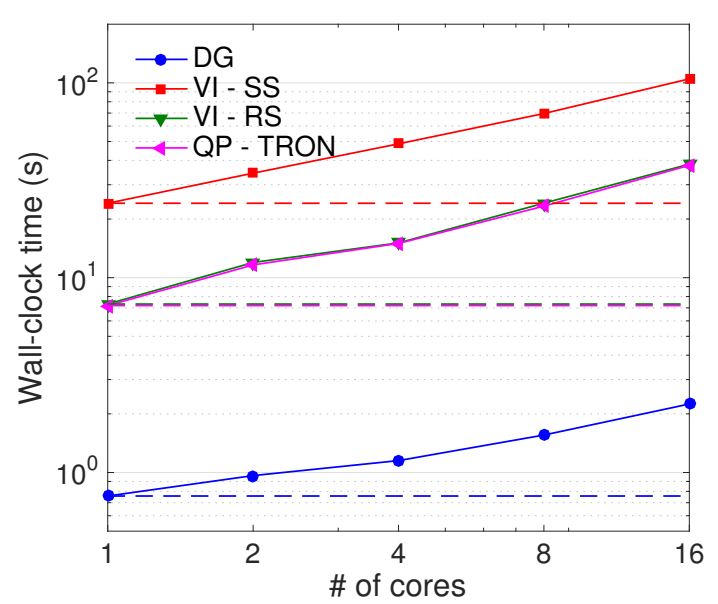

(c) DG - solve time

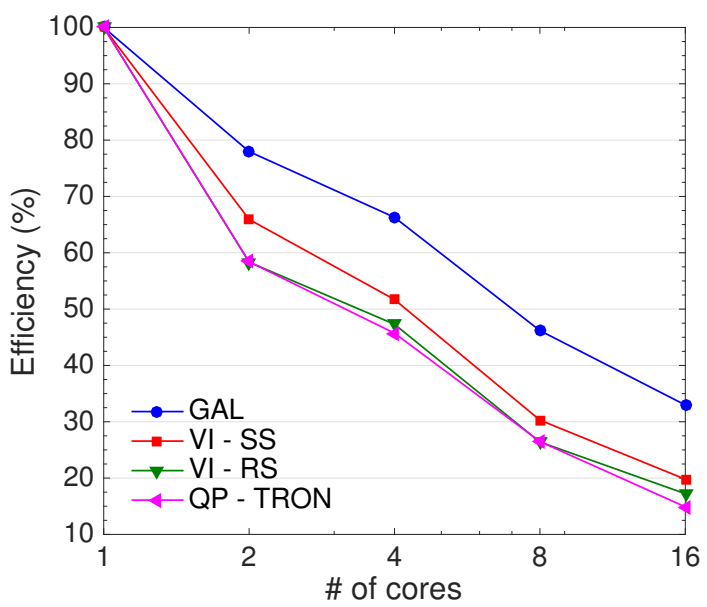

(в) GAL - parallel efficiency

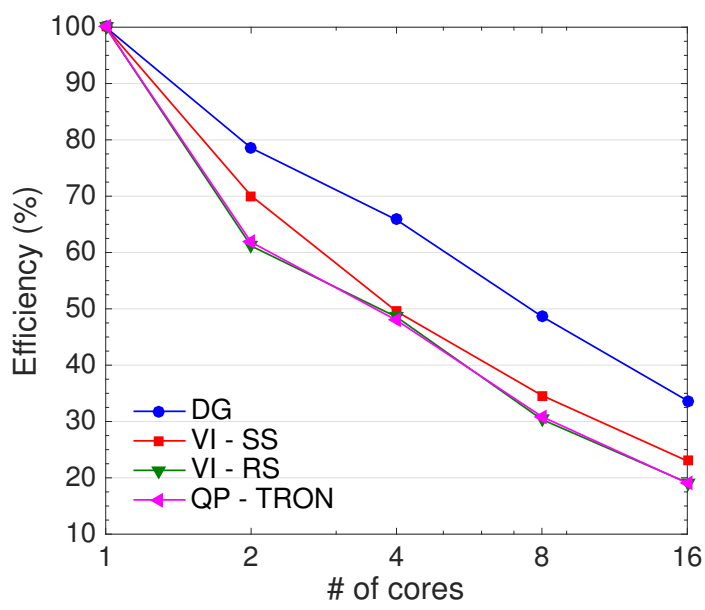

(D) DG - parallel efficiency

FiguRE 12. 3D diffusion: weak-scaling plots with approximately 100k degrees-offreedom per core and the corresponding parallel efficiencies.

sense for two reasons: 1) the wall-clock time and solver iteration for the QP and VI methodologies are not linearly proportional to problem size (increasing solver iterations with $h$-size as seen from Tables 3 and 4), and 2) the lower KSP relative tolerance for the gradient descent computations make the overall solver more sensitive to the memory-bandwidth, meaning that speedup is reduced as the compute nodes become populated with more MPI processes (see Sections 4 and 5 of [Chang et al., 2017] for a more thorough discussion).

However, the weak-scaling plots alone make it difficult to distinguish whether parallel performance deteriorates due to communication overhead or suboptimal algorithmic convergence. To better understand why parallel performance degrades as the number of MPI processes increases, we conduct strong-scaling studies by setting the $h$-size to $1 / 80$ and $1 / 40$ for the GAL and DG formulations respectively (roughly 500k degrees-of-freedom) and study how increasing the number 


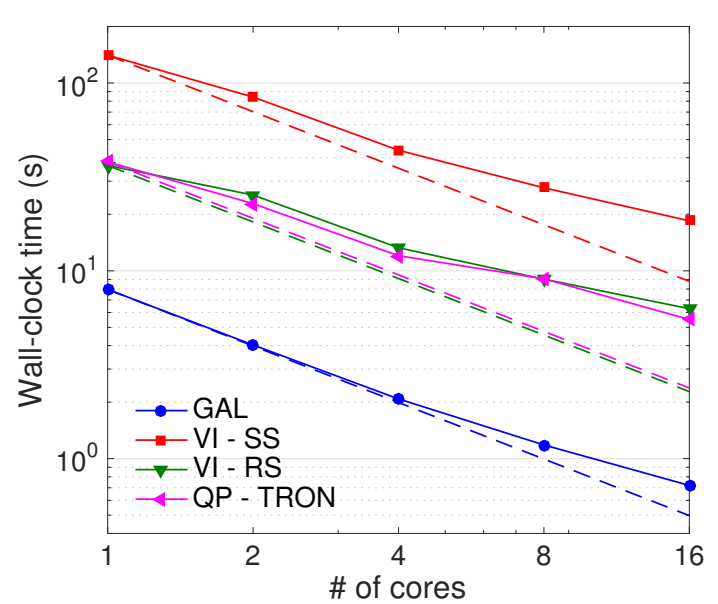

(A) GAL - solver time

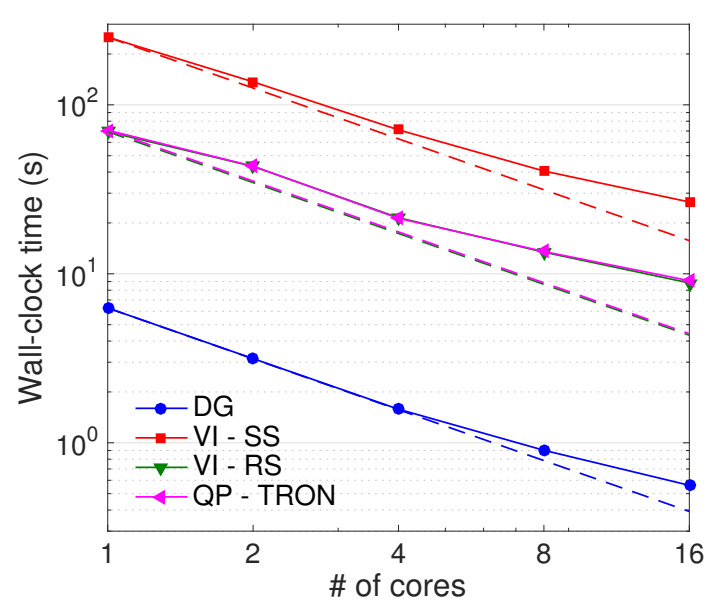

(c) DG - solver time

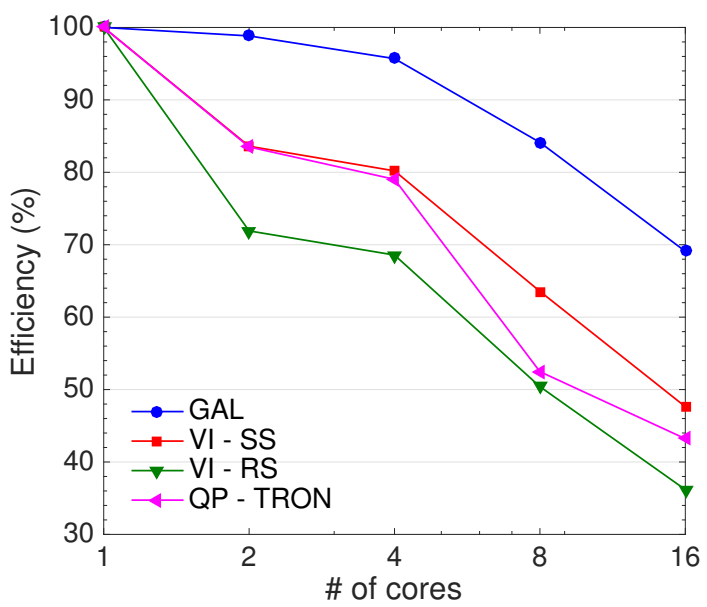

(B) GAL - parallel efficiency

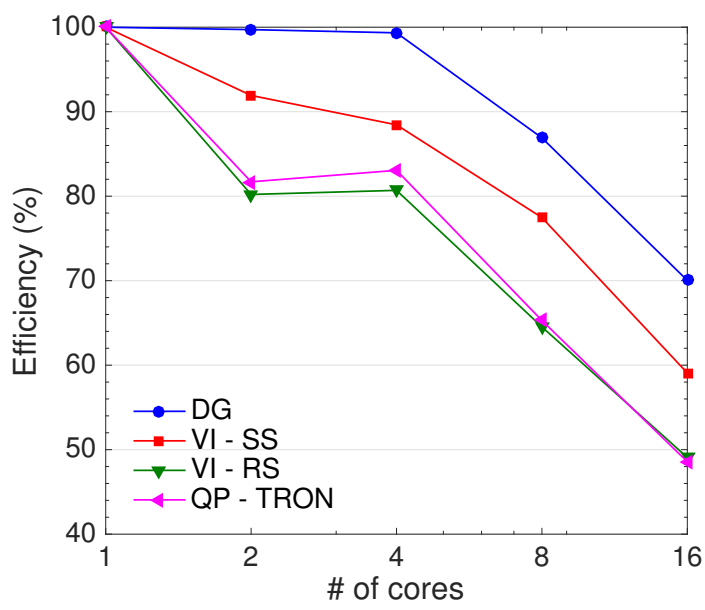

(D) DG - parallel efficiency

FiguRE 13. 3D diffusion: strong-scaling plots for approximately 500k degrees of freedom $(h$-size $=1 / 80$ and $1 / 40$ for GAL and DG respectively) and the corresponding parallel efficiencies.

of MPI processes (hence communication overhead) affects the parallel performance. Figure 13 contains the strong-scaling plots, and we see that the QP and VI solvers still do not scale as well. Regardless of the finite element formulation used, the QP and VI - RS methods have roughly the same strong-scaling performance whereas the VI - SS method has slightly better strong-scaling.

For the advection-diffusion equation, the same problem is considered but advection due to the $\mathrm{ABC}$ flow is now taken into account. A Firedrake project implementation of the DG formulation can be found in Listing 3. Like the diffusion equation, the advection-diffusion equation also exhibits negative concentrations as seen from Figure 14. Table 5 depicts violations under the SUPG formulation to be no greater than $30 \%$ whereas the DG formulation exhibits huge violations as seen from Table 6. Moreover, the single MPI process metrics shown in Tables 7 and 8 clearly indicate that the advection-diffusion equations are generally more expensive to solve than its diffusion counterpart. 


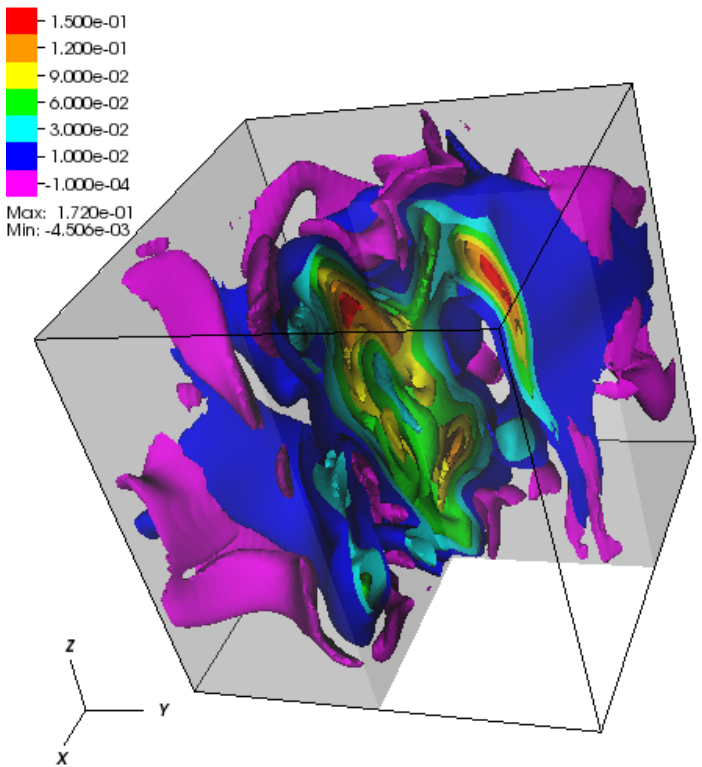

(A) SUPG

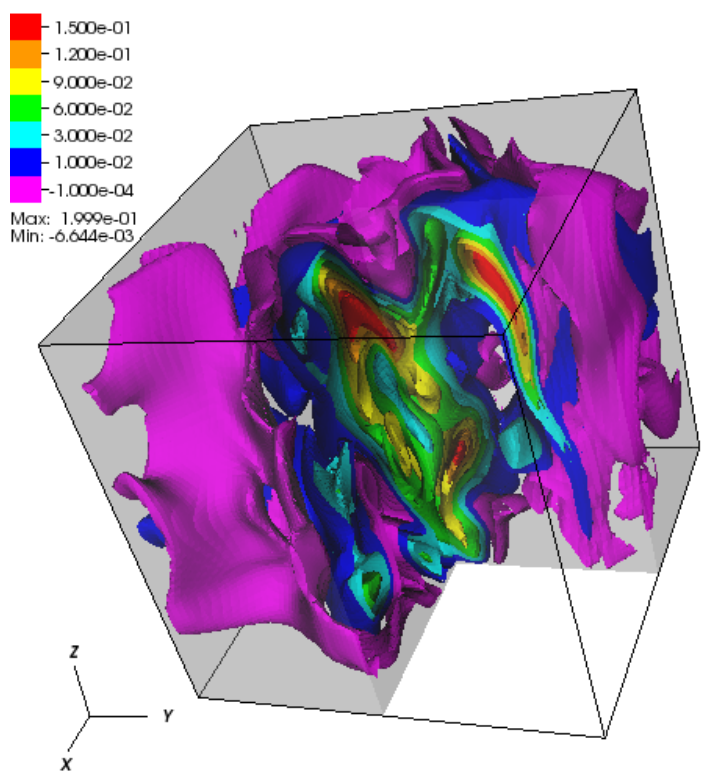

(C) DG

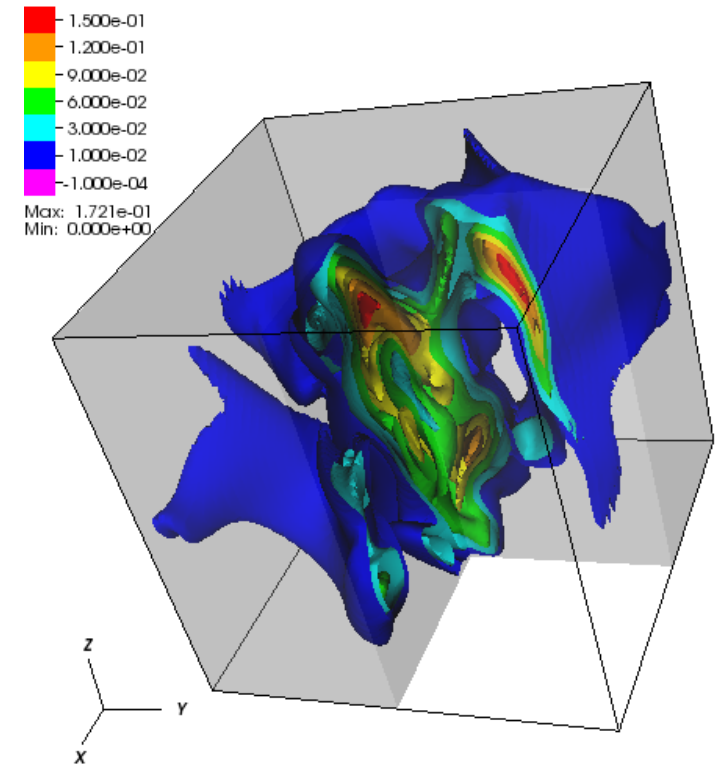

(B) SUPG with VI - SS

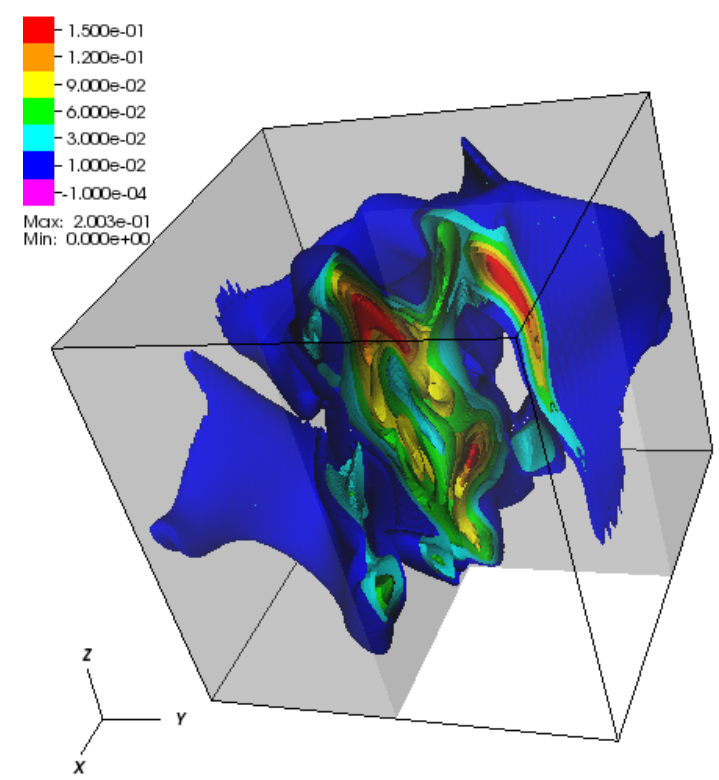

(D) DG with VI - SS

Figure 14. 3D advection-diffusion: 3D contours of the concentrations for the SUPG and DG formulations with and without and VI - SS for $h$-size $=1 / 80$, where the purple contours represent regions with negative concentrations (see online version for color figures.)

These metrics tell us that the VI iterations also begin to stabilize as the problem size increases and that the VI - RS method is faster than the VI - SS method for the advection-diffusion equation. 
TABLE 5. 3D advection-diffusion: minimum and maximum concentrations for various level of mesh refinement under the SUPG formulation.

\begin{tabular}{lccc}
\hline$h$-size & Min. concentration & Max. concentration & \% degrees-of-freedom violated \\
\hline $1 / 10$ & -0.0135676 & 0.187489 & $212 / 1,331 \rightarrow 15.9 \%$ \\
$1 / 20$ & -0.0068733 & 0.180922 & $2,323 / 9,261 \rightarrow 25.1 \%$ \\
$1 / 30$ & -0.0091657 & 0.210942 & $7,964 / 29,791 \rightarrow 26.7 \%$ \\
$1 / 40$ & -0.0055686 & 0.171690 & $18,235 / 68,921 \rightarrow 26.5 \%$ \\
$1 / 50$ & -0.0064795 & 0.185440 & $35,221 / 132,651 \rightarrow 26.6 \%$ \\
$1 / 60$ & -0.0063168 & 0.189047 & $61,171 / 226,981 \rightarrow 26.9 \%$ \\
$1 / 70$ & -0.0053682 & 0.179675 & $99,668 / 357,911 \rightarrow 27.8 \%$ \\
$1 / 80$ & -0.0045065 & 0.172049 & $147,462 / 531,441 \rightarrow 27.7 \%$ \\
\hline
\end{tabular}

TABLE 6. 3D advection-diffusion: minimum and maximum concentrations for various level of mesh refinement under the DG formulation.

\begin{tabular}{lccc}
\hline$h$-size & Min. concentration & Max. concentration & \% degrees-of-freedom violated \\
\hline $1 / 10$ & -0.0151514 & 0.259127 & $4,976 / 8,000 \rightarrow 62.2 \%$ \\
$1 / 20$ & -0.0162537 & 0.211295 & $37,464 / 64,000 \rightarrow 58.5 \%$ \\
$1 / 30$ & -0.0137824 & 0.237722 & $120,296 / 216,000 \rightarrow 55.7 \%$ \\
$1 / 40$ & -0.0067079 & 0.186956 & $276,832 / 512,000 \rightarrow 54.1 \%$ \\
$1 / 50$ & -0.0057574 & 0.203852 & $526,080 / 1,000,000 \rightarrow 52.6 \%$ \\
$1 / 60$ & -0.0065627 & 0.203093 & $891,768 / 1,728,000 \rightarrow 51.6 \%$ \\
$1 / 70$ & -0.0069389 & 0.193418 & $1,410,208 / 2,744,000 \rightarrow 51.4 \%$ \\
$1 / 80$ & -0.0066445 & 0.199912 & $2,069,752 / 4,096,000 \rightarrow 50.5 \%$ \\
\hline
\end{tabular}

The weak-scaling plots, as seen from Figure 15, indicate that the VI solvers are identical in performance, but the strong-scaling plots from Figure 16 suggest that VI - SS has slightly better parallel speedup than VI - RS. These steady-state numerical experiments suggest that the VI - RS is the preferred methodology for solving large-scale advection-diffusion equations because it takes less time to solve than other more expensive VI algorithms like VI - SS. However, if advection becomes negligible thus reducing the system to a symmetric diffusion problem, one could employ either QP - TRON or VI - RS as these solvers are equal in both parallel and algorithmic scalability.

5.3. Solver tolerances. So far the results in this section suggest that the VI approach is a fairly computationally expensive for both diffusion and advection-diffusion type equations. This is, partly, because we used a stringent solver convergence criterion (an absolute residual tolerance of $10^{-8}$ ) for both QP and VI. The exact choice of tolerance is indeed problem and application dependent, but our proposed computational framework sets the solution to the unconstrained problem as the initial guess. Therefore, we have the luxury to use a more relaxed solver convergence criterion and investigate how a relative residual tolerance of $10^{-1}$ affects the performance of the VI solvers numerically and algorithmically. Table 9 contains the time-to-solution and the total number of VI iterations for the advection-diffusion problem under the SUPG and DG formulations. Relaxing the tolerance greatly reduces the amount of wall-clock time and solver iterations needed 
TABLE 7. 3D advection-diffusion: wall-clock time and number of linear (KSP) or nonlinear (VI) solve iterations for various levels of mesh refinement under the SUPG formulation.

\begin{tabular}{l|cc|cc|cc}
\hline \multirow{2}{*}{$h$-size } & \multicolumn{2}{|c|}{ SUPG } & \multicolumn{2}{c|}{ VI - SS } & \multicolumn{2}{c}{ VI - RS } \\
& time (s) & iters & time (s) & iters & time (s) & iters \\
\hline $1 / 10$ & 0.003 & 9 & 0.028 & 5 & 0.009 & 2 \\
$1 / 20$ & 0.045 & 16 & 0.504 & 11 & 0.146 & 4 \\
$1 / 30$ & 0.221 & 22 & 2.525 & 14 & 0.710 & 5 \\
$1 / 40$ & 0.768 & 29 & 10.21 & 20 & 2.592 & 6 \\
$1 / 50$ & 2.019 & 35 & 27.27 & 23 & 8.842 & 10 \\
$1 / 60$ & 4.530 & 43 & 65.61 & 30 & 19.20 & 10 \\
$1 / 70$ & 8.178 & 47 & 117.3 & 28 & 34.54 & 11 \\
$1 / 80$ & 14.70 & 55 & 229.4 & 34 & 66.07 & 12 \\
\hline
\end{tabular}

TABLE 8. 3D advection-diffusion: wall-clock time and number of linear (KSP) or nonlinear (VI) solve iterations for various levels of mesh refinement under the DG formulation.

\begin{tabular}{l|cc|cc|cc}
\hline \multirow{2}{*}{$h$-size } & \multicolumn{2}{|c|}{ DG } & \multicolumn{2}{c|}{ VI - SS } & \multicolumn{2}{c}{ VI - RS } \\
& time (s) & iters & time (s) & iters & time (s) & iters \\
\hline $1 / 10$ & 0.031 & 10 & 0.807 & 13 & 0.202 & 5 \\
$1 / 20$ & 0.459 & 14 & 14.46 & 21 & 3.890 & 8 \\
$1 / 30$ & 2.352 & 19 & 73.84 & 27 & 19.56 & 10 \\
$1 / 40$ & 6.819 & 22 & 251.3 & 35 & 56.94 & 11 \\
$1 / 50$ & 15.49 & 25 & 629.1 & 40 & 137.1 & 13 \\
$1 / 60$ & 30.65 & 28 & 1387 & 47 & 292.3 & 15 \\
$1 / 70$ & 57.56 & 32 & 2267 & 51 & 570.0 & 18 \\
$1 / 80$ & 97.15 & 36 & 7105 & 60 & 917.2 & 18 \\
\hline
\end{tabular}

with acceptable compromise on the accuracy as seen from the absolute difference in concentrations in Figure 17. The weak scaling plots in Figures 18 indicate that the VI - SS has relatively good algorithmic performance with respect to problem size. whereas the strong-scaling plots in Figure 19 still indicate that VI - RS is not as scalable as the VI - RS.

REMARK 2. These parallel performance studies do not indicate how truly efficient the PETSc and TAO implementations of the QP and VI solvers are in the context of high performance computing. It should be noted that a serially efficient algorithm will likely have poor parallel performance due to dominating effects from communication overhead and memory latencies. Moreover, the use of assembled sparse matrices coupled with memory bandwidth effects explains why the scaling can degrade even when only 2 MPI processes are used. A more thorough discussion on memory bandwidth limitations as well as a roofline-like hardware performance model based on the perfect cache model can be found in Section 4 of [Chang et al., 2017] and the references within. 


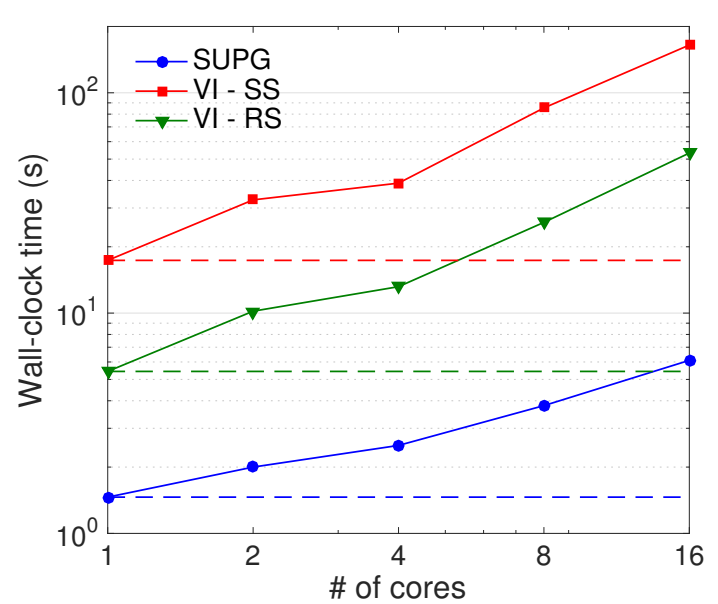

(A) SUPG - solve time

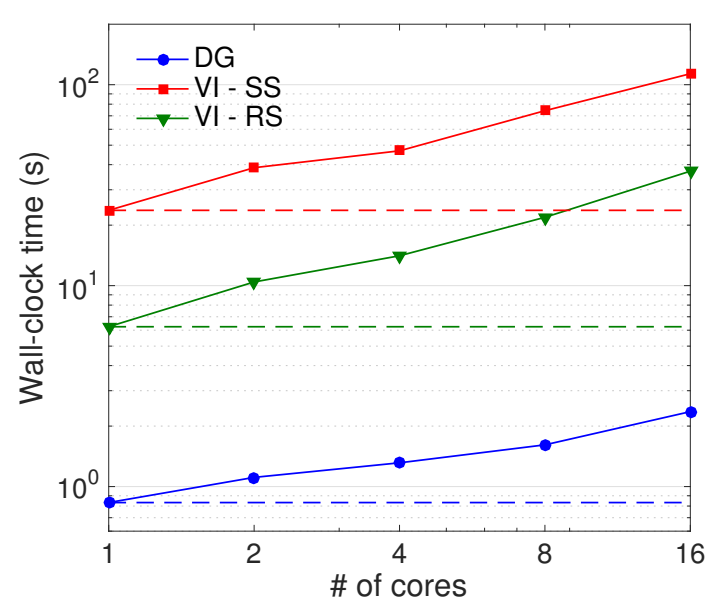

(c) DG - solve time

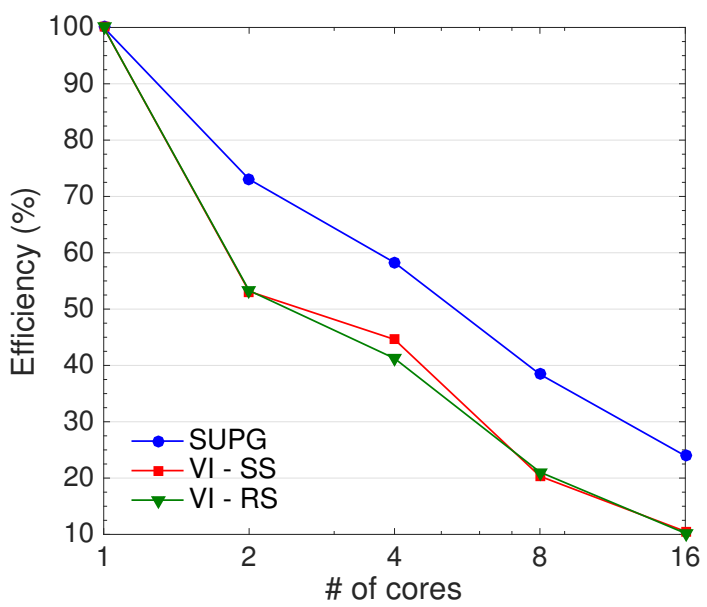

(в) SUPG - parallel efficiency

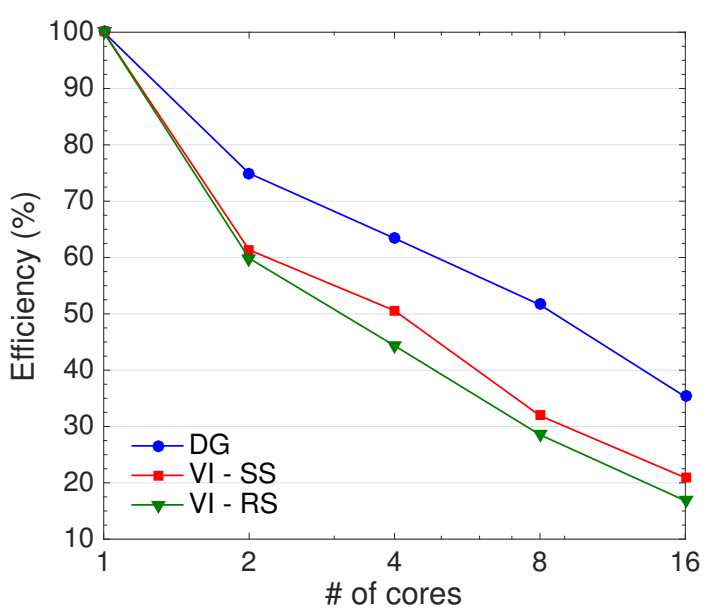

(D) DG - parallel efficiency

FigURE 15. 3D advection-diffusion: weak-scaling plots with approximately 100k degrees-of-freedom per core and the corresponding parallel efficiencies.

\section{EXTENSION TO TRANSIENT ANALYSIS AND COUPLED PROBLEMS}

We now illustrate that the proposed framework, which is based on variational inequalities, can be extended to perform a transient analysis. The resulting governing equations will then be parabolic variational inequalities. This extension will be illustrated by considering the displacement of miscible fluids in porous media wherein a fluid displaces a fluid with higher viscosity [Stalkup, 1983]. Some of the applications of miscible displacement include oil recovery and carbon-dioxide sequestration [Chen and Meiburg, 1998a,b]. The phenomenon is commonly modeled using coupled flow and transport equations, which will be presented below. In this section, we will also show how negative concentrations can have serious ramifications when simulating non-linear transport phenomenon like the displacement of miscible fluids. 


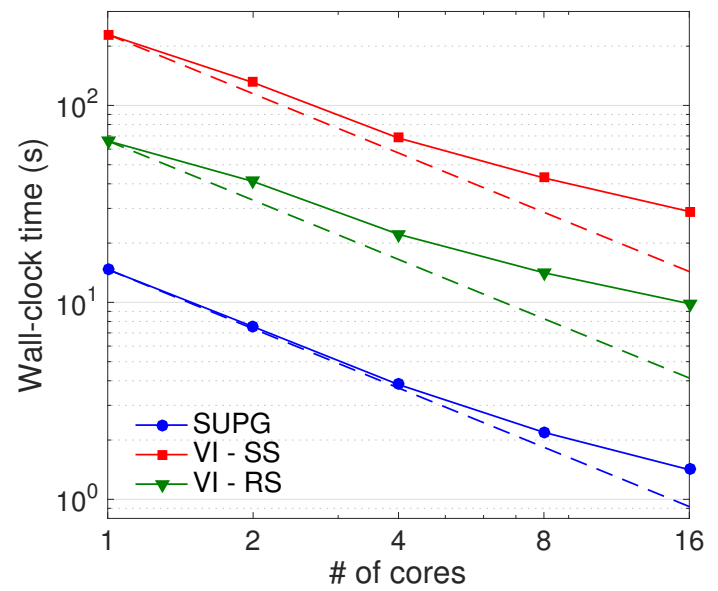

(A) SUPG - solve time

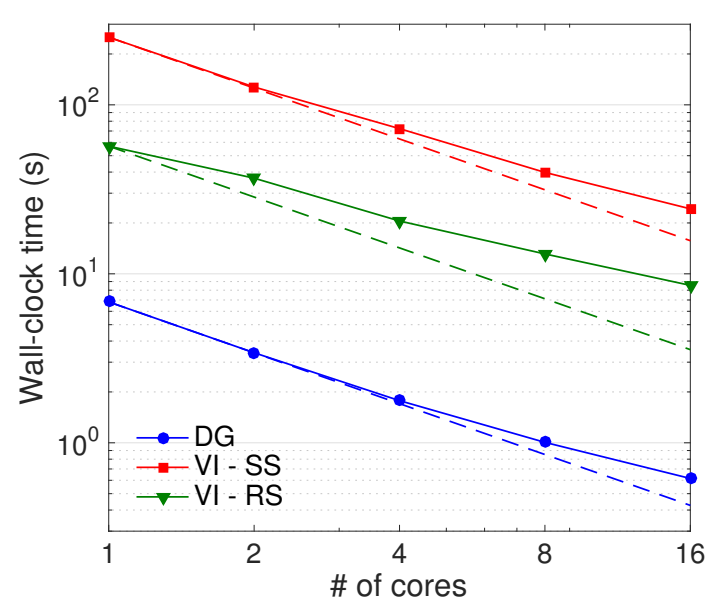

(C) DG - solve time

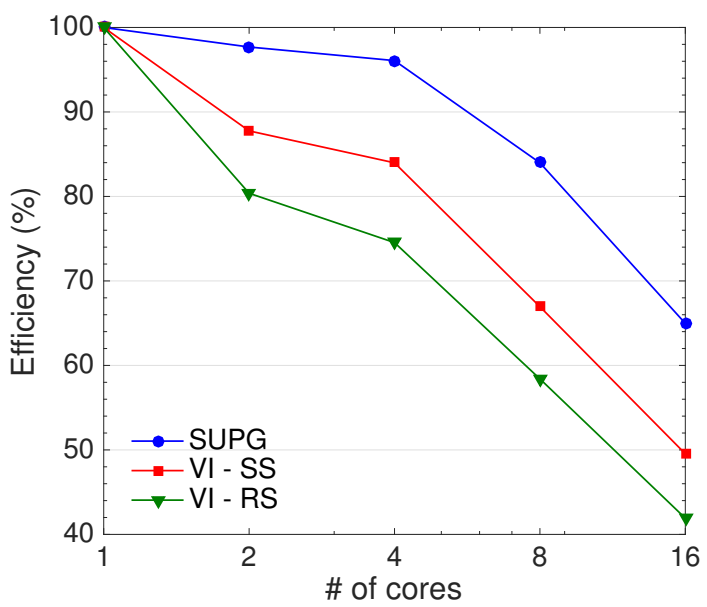

(B) SUPG - parallel efficiency

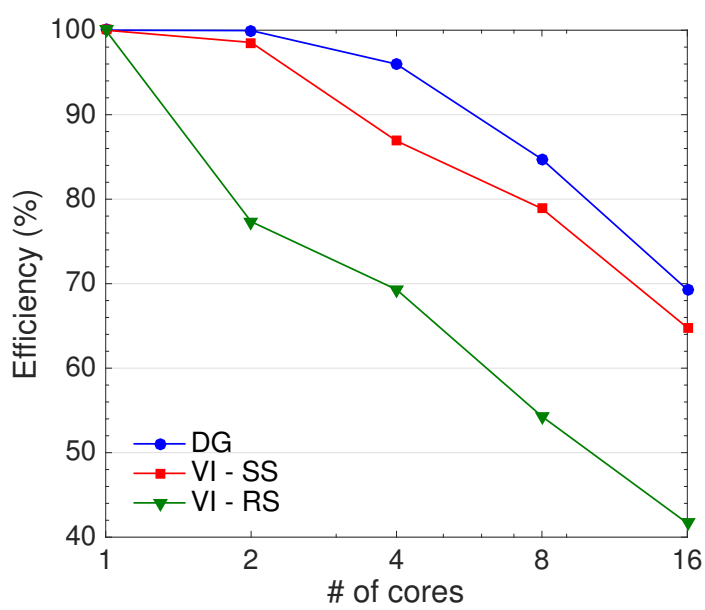

(D) DG - parallel efficiency

FigURE 16. 3D advection-diffusion: strong-scaling plots for approximately 500k degrees of freedom ( $h$-size $=1 / 80$ and $1 / 40$ for GAL and DG respectively) and the corresponding parallel efficiencies.

6.1. Governing equations and temporal discretization. We denote the time by $t \in$ $[0, \mathcal{T}]$, where $\mathcal{T}$ denotes the length of the time interval of interest. For the Darcy equation, the boundary is divided into two parts: $\Gamma^{p}$ and $\Gamma^{v}$, such that $\Gamma^{p} \cup \Gamma^{v}=\partial \Omega$ and $\Gamma^{p} \cap \Gamma^{v}=\emptyset$. $\Gamma^{p}$ and $\Gamma^{v}$ denote the parts of the boundary on which pressure and velocity boundary conditions are enforced respectively. We shall denote time-dependent pressure by $p(\mathbf{x}, t)$, time-dependent velocity by $\mathbf{v}(\mathbf{x}, t)$, concentration-dependent viscosity by $\mu(c(\mathbf{x}, t))$, permeability by $k(\mathbf{x})$, density by $\rho$, timedependent specific body force by $\mathbf{b}(\mathbf{x}, t)$, time-dependent concentration by $c(\mathbf{x}, t)$, prescribed initial concentration by $c_{0}(\mathbf{x})$, time dependent volumetric source by $f(\mathbf{x}, t)$, and time-dependent diffusivity tensor by $\mathbf{D}(\mathbf{x}, t)$. For the boundary conditions, the prescribed time-dependent concentration is denoted by $c^{\mathrm{p}}(\mathbf{x}, t)$, prescribed time-dependent pressure by $p^{\mathrm{P}}(\mathbf{x}, t)$, prescribed time-dependent normal component of the velocity by $v_{n}(\mathbf{x}, t)$, and prescribed time-dependent flux by $q^{\mathrm{P}}(\mathbf{x}, t)$. The 
TABLE 9. 3D advection-diffusion: wall-clock time and number of VI solve iterations for various levels of mesh refinement when the relative solver tolerance is set to $10^{-1}$.

\begin{tabular}{l|cc|cc|cc|cc}
\hline \multirow{2}{*}{$h$-size } & \multicolumn{2}{|c|}{ SUPG: VI - SS } & \multicolumn{2}{c|}{ SUPG: VI - RS } & \multicolumn{2}{c|}{ DG: VI - SS } & \multicolumn{2}{c}{ DG: VI - RS } \\
& time (s) & iters & time (s) & iters & time (s) & iters & time (s) & iters \\
\hline $1 / 10$ & 0.051 & 2 & 0.005 & 1 & 0.653 & 2 & 0.088 & 2 \\
$1 / 20$ & 0.376 & 2 & 0.076 & 2 & 5.471 & 2 & 1.512 & 3 \\
$1 / 30$ & 1.316 & 2 & 0.315 & 2 & 19.64 & 2 & 6.693 & 3 \\
$1 / 40$ & 4.425 & 3 & 1.308 & 3 & 48.70 & 2 & 18.20 & 3 \\
$1 / 50$ & 6.723 & 2 & 3.125 & 3 & 97.53 & 2 & 50.98 & 4 \\
$1 / 60$ & 11.80 & 2 & 6.232 & 3 & 174.2 & 2 & 96.47 & 4 \\
$1 / 70$ & 19.68 & 2 & 14.92 & 4 & 290.0 & 2 & 196.6 & 5 \\
$1 / 80$ & 30.83 & 2 & 24.73 & 4 & 430.1 & 2 & 305.6 & 5 \\
\hline
\end{tabular}

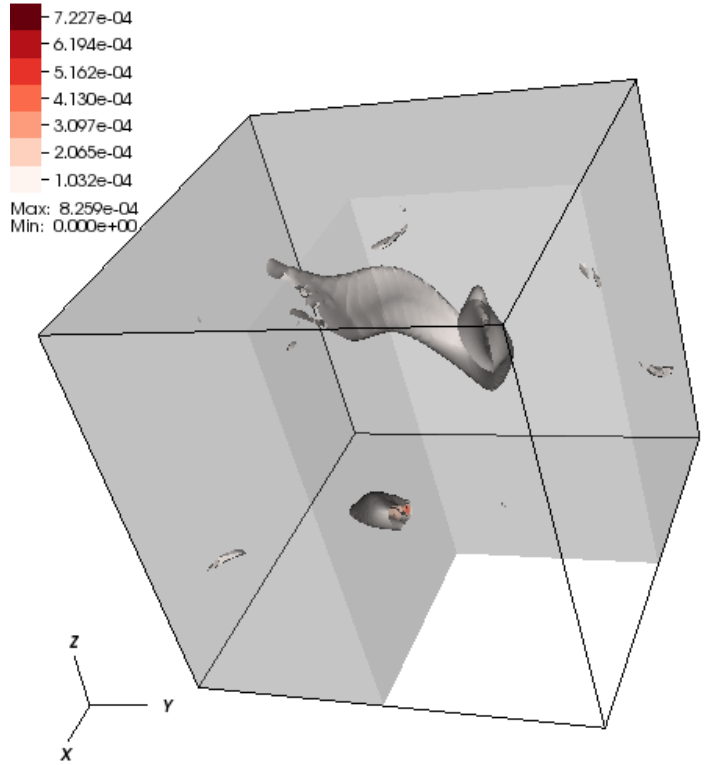

(A) SUPG

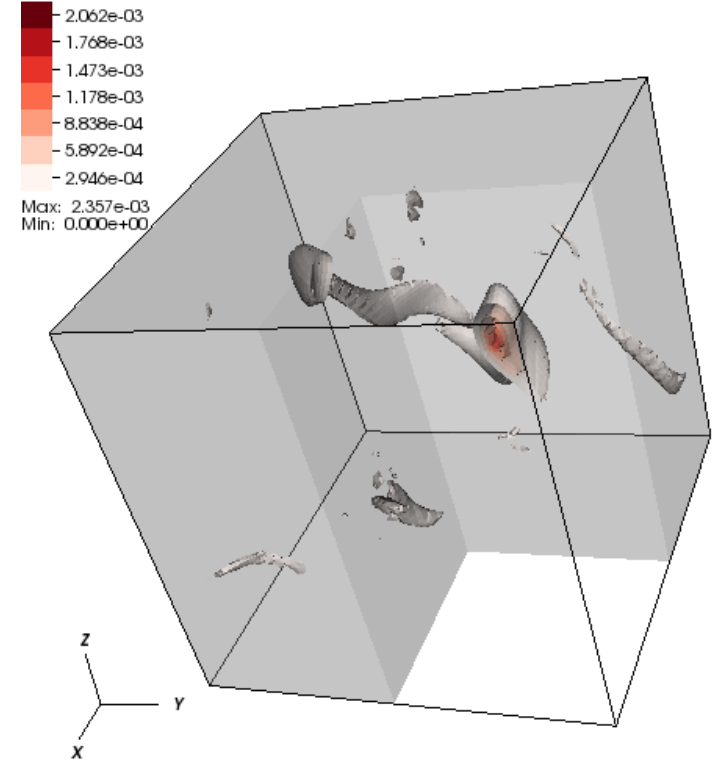

(B) DG

FiguRE 17. 3D advection-diffusion: 3D contours of the absolute difference in concentrations between the VI - RS solution that converged at a relative residual tolerance of $10^{-1}$ and the VI - RS solution that converged at an absolute residual tolerance of $10^{-8}$.

initial boundary value problem for the coupled flow and advective-diffusive equations can be written as follows:

$$
\begin{aligned}
& \frac{\mu(c(\mathbf{x}, t))}{k(\mathbf{x})} \mathbf{v}(\mathbf{x}, t)+\operatorname{grad}[p(\mathrm{x}, t)]=\rho \mathbf{b}(\mathbf{x}, t) \\
& \operatorname{div}[\mathbf{v}(\mathbf{x}, t)]=0 \\
& p(\mathbf{x}, t)=p^{\mathrm{P}}(\mathbf{x}, t)
\end{aligned}
$$

$$
\begin{aligned}
& \text { in } \Omega \times(0, \mathcal{T}) \\
& \text { in } \Omega \times(0, \mathcal{T}) \\
& \text { on } \Gamma^{p} \times(0, \mathcal{T})
\end{aligned}
$$




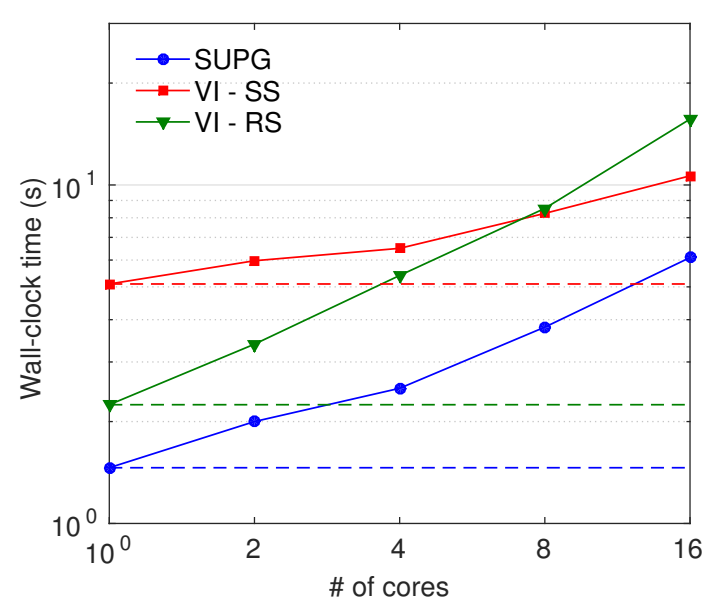

(A) SUPG - solve time

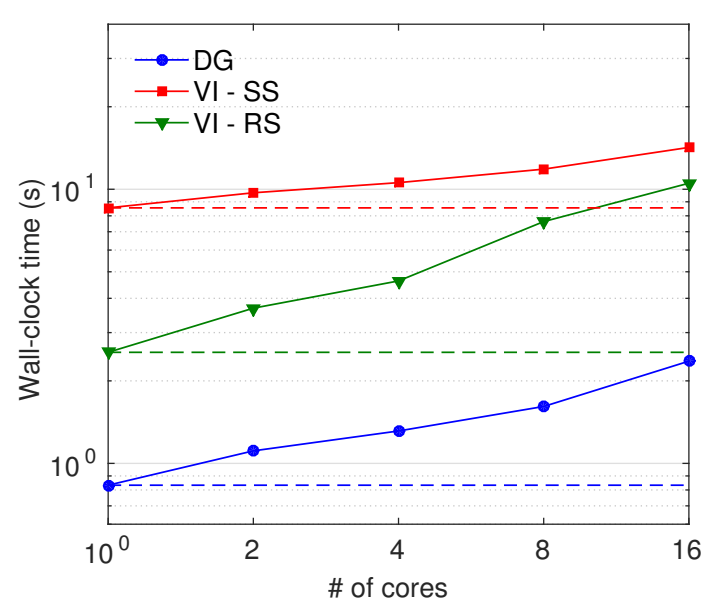

(c) DG - solve time

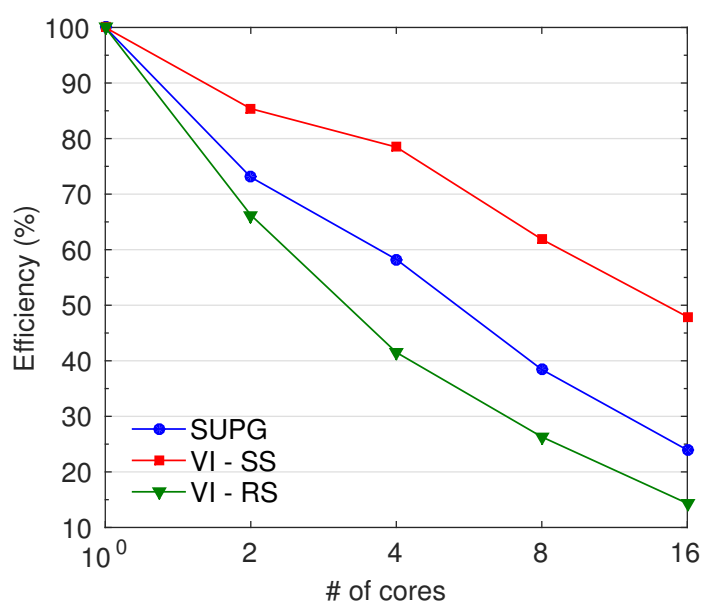

(в) SUPG - parallel efficiency

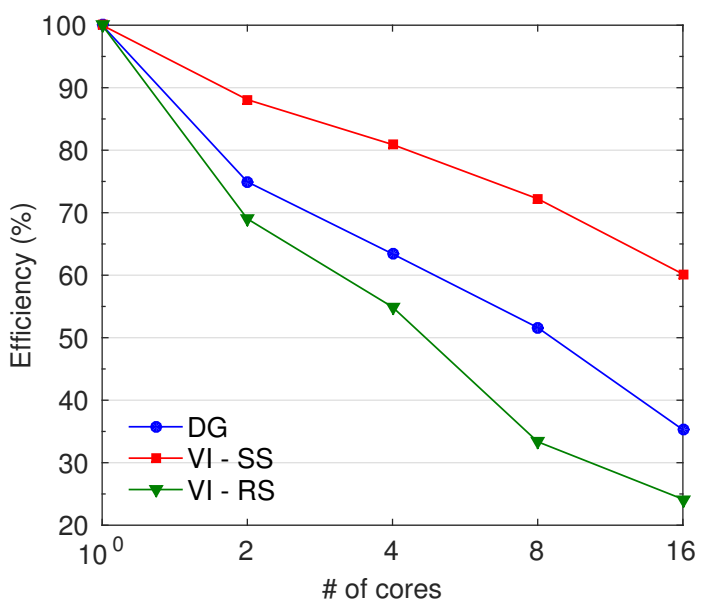

(D) DG - parallel efficiency

FigURE 18. 3D advection-diffusion: weak-scaling plots of the relaxed VI solvers with approximately 100k degrees-of-freedom per core and the corresponding parallel efficiencies.

$$
\begin{array}{ll}
\mathbf{v}(\mathbf{x}, t) \cdot \widehat{\mathbf{n}}=v_{n}(\mathbf{x}, t) & \text { on } \Gamma^{v} \times(0, \mathcal{T}) \\
\frac{\partial c(\mathbf{x}, t)}{\partial t}+\mathbf{v}(\mathbf{x}, t) \cdot \operatorname{grad}[c(\mathbf{x}, t)]-\operatorname{div}[\mathbf{D}(\mathbf{x}, t) \operatorname{grad}[c(\mathbf{x}, t)]]=f(\mathbf{x}, t) & \text { in } \Omega \times(0, \mathcal{T}) \\
c(\mathbf{x}, t)=c^{\mathrm{P}}(\mathbf{x}, t) & \text { on } \Gamma^{\mathrm{D}} \times(0, \mathcal{T}) \\
\widehat{\mathbf{n}}(\mathbf{x}) \cdot(\mathbf{v}(\mathbf{x}, t) c(\mathbf{x}, t)-\mathbf{D}(\mathbf{x}, t) \operatorname{grad}[c(\mathbf{x}, t)])=q^{\mathrm{p}}(\mathbf{x}, t) & \text { on } \Gamma_{\text {inflow }}^{\mathrm{N}} \times(0, \mathcal{T}) \\
-\widehat{\mathbf{n}}(\mathbf{x}) \cdot \mathbf{D}(\mathbf{x}, t) \operatorname{grad}[c(\mathbf{x}, t)]=q^{\mathrm{P}}(\mathbf{x}, t) & \text { on } \Gamma_{\text {outflow }}^{\mathrm{N}} \times(0, \mathcal{T}) \\
c(\mathbf{x}, 0)=c_{0}(\mathbf{x}) & \text { in } \Omega
\end{array}
$$

where equations (6.1a) through (6.1d) represent the Darcy equation, and equations (6.1e) through (6.1i) represent the transient advection-diffusion equation. To complete the coupled problem, the 


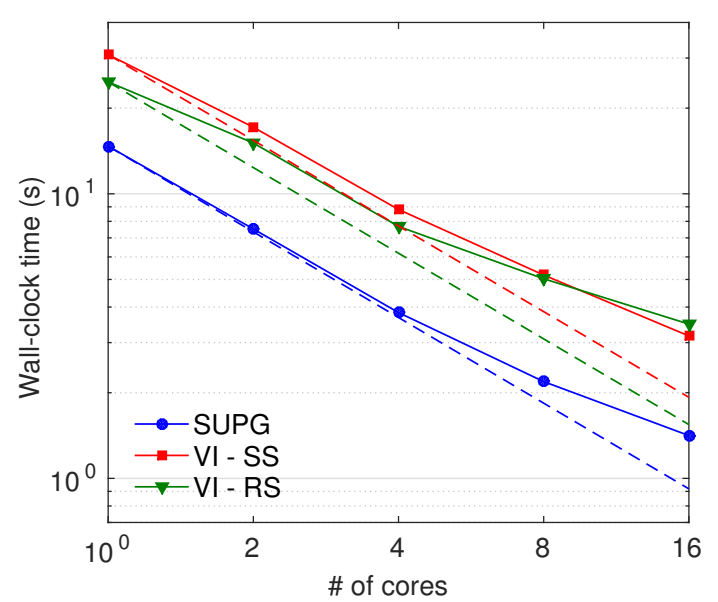

(A) SUPG - solve time

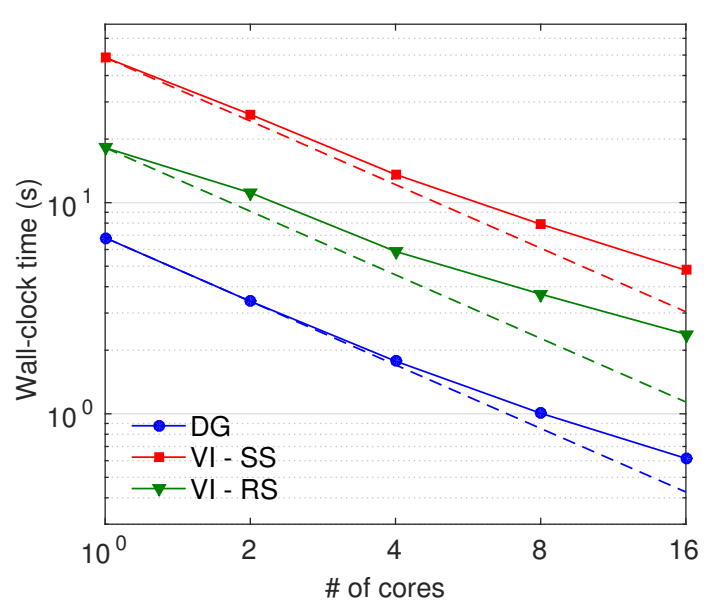

(C) DG - solve time

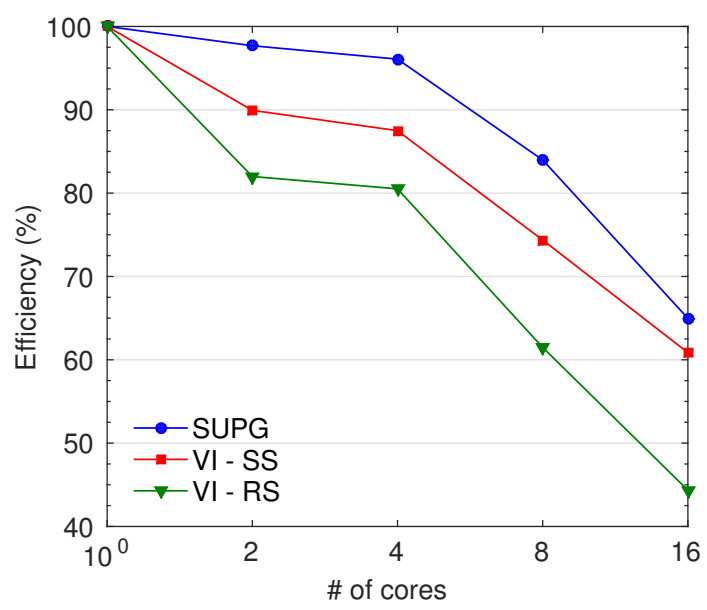

(B) SUPG - parallel efficiency

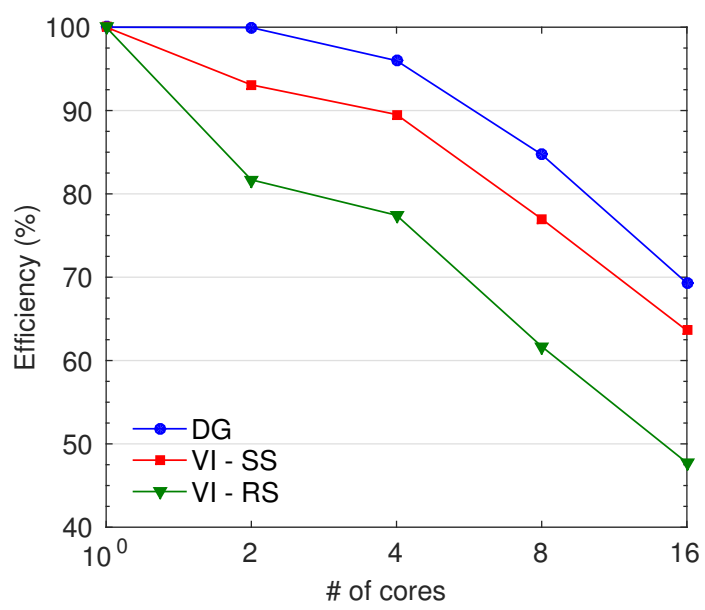

(D) DG - parallel efficiency

FIGURE 19. 3D advection-diffusion: strong-scaling plots of the relaxed VI solvers for approximately $500 \mathrm{k}$ degrees of freedom $(h$-size $=1 / 80$ and $1 / 40$ for GAL and DG respectively) and the corresponding parallel efficiencies.

viscosity is assumed to depend exponentially on concentration:

$$
\begin{aligned}
& \mu(c(\mathbf{x}, t))=\mu_{0} \exp \left[R_{c} c(\mathbf{x}, t)\right] \\
& \mu(c(\mathbf{x}, t))=\mu_{0} \exp \left[R_{c}(1-c(\mathbf{x}, t))\right]
\end{aligned}
$$

where $\mu_{0}$ is the base viscosity of the less viscous fluid and $R_{c}$ is the log-mobility ratio in an isothermal miscible displacement. 


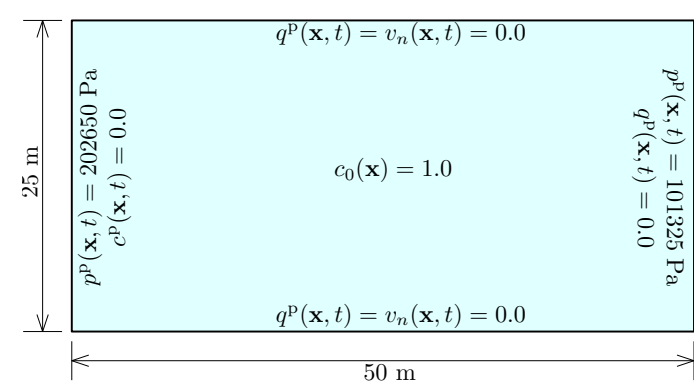

(A) Problem description

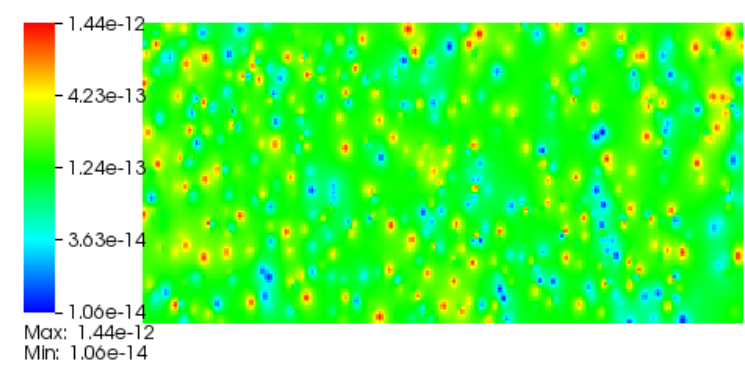

(B) $\log$ scale permeability field $\left(\mathrm{m}^{2}\right)$

Figure 20. 2D miscible displacement: Pictorial description of the boundary value problems for the coupled Darcy and advection-diffusion equations and the corresponding random permeability.

To solve the transient advection-diffusion equation, we employ the method of horizontal lines [Grossmann et al., 2007], which first discretizes the time derivatives, thereby giving rise to timeindependent equations. The time interval of interest is divided into $N$ sub-intervals. That is,

$$
[0, \mathcal{T}]:=\bigcup_{n=0}^{N}\left[t_{n}, t_{n+1}\right]
$$

where $t_{n}$ denotes the $n$-th time-level. We assume that the time-step is uniform, which can be written as:

$$
\Delta t=t_{n+1}-t_{n}
$$

One can then employ the finite-dimensional VI solvers for these resulting equations, which were described earlier in this paper. This implies that we will still be solving elliptic VIs of first kind but at each time level. This procedure will be illustrated below using the backward Euler method.

TABLE 10. 2D miscible displacement: problem parameters

\begin{tabular}{ll}
\hline Parameter & Value \\
\hline $\mathbf{b}(\boldsymbol{x})$ & $\{0,0\} \mathrm{m} / \mathrm{s}^{2}$ \\
$\mu(c(\mathbf{x}, t))$ & See equation $(6.2 \mathrm{a})$ \\
$\mu_{0}$ & $3.95 \cdot 10^{-5} \mathrm{~Pa} \mathrm{~s}$ \\
$R_{c}$ & 3 \\
$k(\mathbf{x})$ & varies \\
$f(\mathbf{x}, t)$ & 0 \\
$\rho$ & $479 \mathrm{~kg} / \mathrm{m}^{3}$ \\
$\alpha_{L}$ & $10^{-1} \mathrm{~m}$ \\
$\alpha_{T}$ & $10^{-5} \mathrm{~m}$ \\
$\alpha_{D}$ & $10^{-9} \mathrm{~m}^{2} / \mathrm{s}$ \\
Number of elements & 31,250 \\
Darcy degrees-of-freedom & 94,125 \\
Advection-diffusion degrees-of-freedom & 125,000 \\
\hline
\end{tabular}




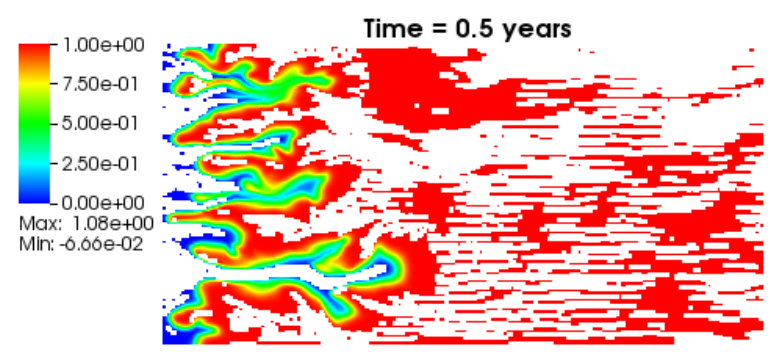

(A) $\boldsymbol{c}_{\mathrm{DG}}$ at $t=0.5$ years

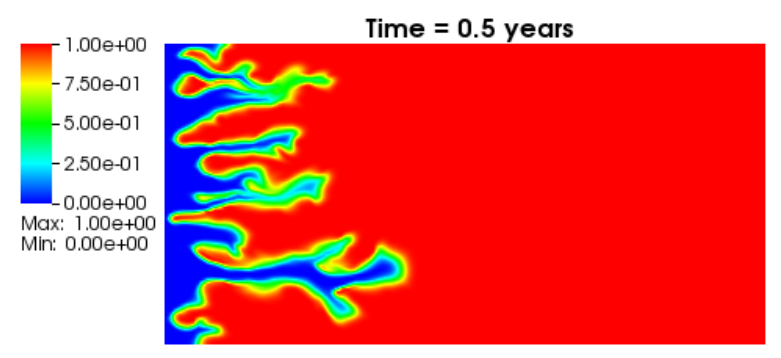

(C) $\boldsymbol{c}_{\mathrm{RS}}$ at $t=0.5$ years

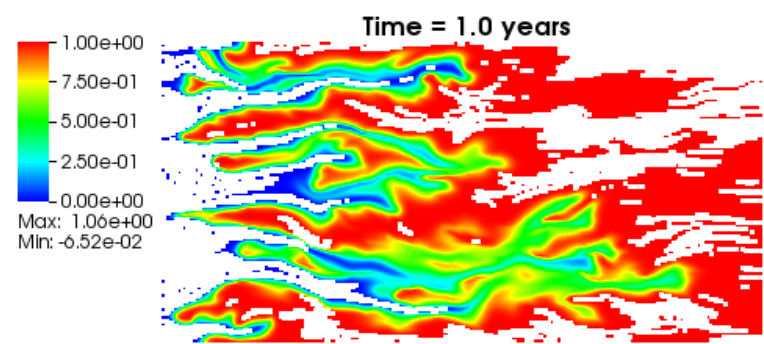

(B) $\boldsymbol{c}_{\mathrm{DG}}$ at $t=1.0$ years

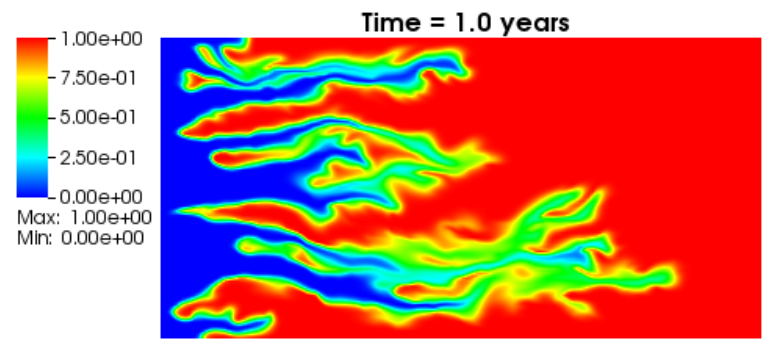

(D) $\boldsymbol{c}_{\mathrm{RS}}$ at $t=1.0$ years

Figure 21. 2D miscible displacement: Concentration fields under the DG $\left(\boldsymbol{c}_{\mathrm{DG}}\right)$ formulation and VI - RS $\left(\boldsymbol{c}_{\mathrm{RS}}\right)$ method at various time levels. White regions denote violations of the maximum principle and the non-negative constraint.

However, a detailed discussion on the effect of time-stepping schemes in meeting maximum principles can be found in [Nakshatrala et al., 2016]. For a transient analysis, the proposed framework outlined in Section 4.2.2 is modified as follows:

Step 1: Set $t=0.0, n=0$, and $\boldsymbol{c}^{(n)}=\boldsymbol{c}_{0}$.

Step 2: Solve Darcy equation:

(a) Compute $\mu\left(\boldsymbol{c}^{(n)}\right)$ using equation (6.2).

(b) Assemble $\boldsymbol{K}_{v v}, \boldsymbol{K}_{v p}, \boldsymbol{K}_{p v}, \boldsymbol{K}_{p p}, \boldsymbol{f}_{v}$ and $\boldsymbol{f}_{p}$.

(c) Solve for $\boldsymbol{v}^{(n)}$.

Step 3: Solve advection-diffusion equation:

(a) Compute $\mathbf{D}^{(n)}$ using equation (5.3).

(b) Assemble $\boldsymbol{K}_{c}$ and $\boldsymbol{f}_{c}$ using $\boldsymbol{c}^{(n)}$.

(c) Solve for $\boldsymbol{c}_{\mathrm{DG}}^{(n+1)}$.

(d) Clip $\boldsymbol{c}_{\mathrm{DG}}^{(n+1)}$ and obtain $\boldsymbol{c}_{\mathrm{CLIP}}^{(n+1)}$.

(e) Solve the bounded constraint problem for $\boldsymbol{c}_{\mathrm{RS}}^{(n+1)}$ with $\boldsymbol{c}_{\mathrm{CLIP}}^{(n+1)}$ as the initial guess.

Step 4: Set $\boldsymbol{c}^{(n+1)} \longleftarrow c_{\mathrm{RS}}^{(n+1)}, t \longleftarrow t+\Delta t$, and $n \longleftarrow n+1$.

Step 5: If $n<N$ go to Step2.

where $\boldsymbol{K}_{v v}, \boldsymbol{K}_{v p}, \boldsymbol{K}_{p v}, \boldsymbol{K}_{p p}, \boldsymbol{f}_{v}$, and $\boldsymbol{f}_{p}$ are the assembled matrices and vectors for the Darcy equation, and $\boldsymbol{K}_{c}$ and $\boldsymbol{f}_{c}$ are for the transient advection-diffusion equation. The finite element 


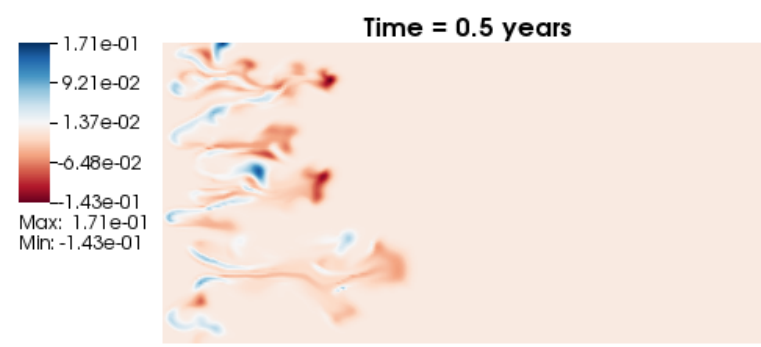

(A) $\boldsymbol{c}_{\mathrm{CLIP}}-\boldsymbol{c}_{\mathrm{RS}}$ at $t=0.5$ years

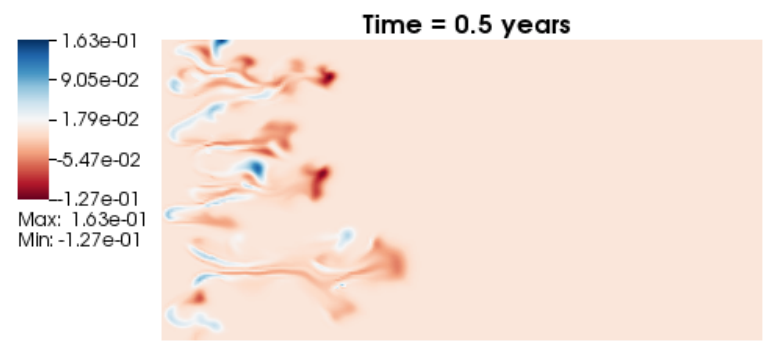

(C) $\boldsymbol{c}_{\mathrm{DG}}-\boldsymbol{c}_{\mathrm{RS}}$ at $t=0.5$ years

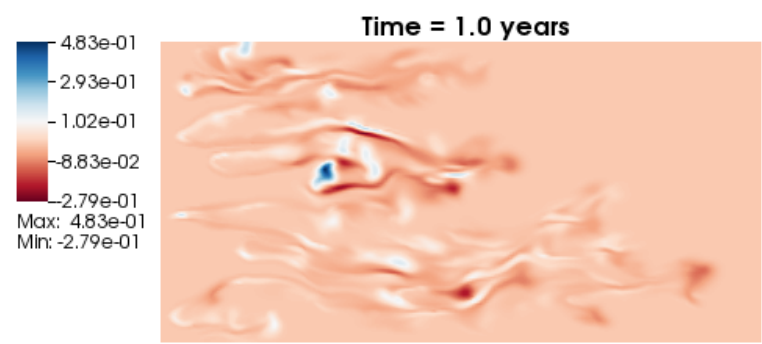

(B) $\boldsymbol{c}_{\mathrm{CLIP}}-\boldsymbol{c}_{\mathrm{RS}}$ at $t=1.0$ years

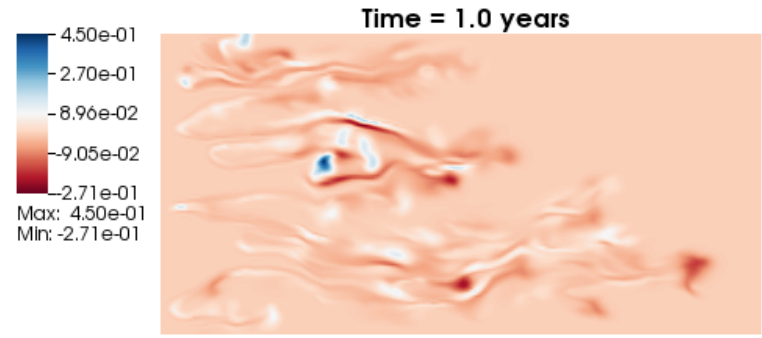

(D) $\boldsymbol{c}_{\mathrm{DG}}-\boldsymbol{c}_{\mathrm{RS}}$ at $t=1.0$ years

Figure 22. 2D miscible displacement: Differences between concentration fields under the DG formulation $\left(\boldsymbol{c}_{\mathrm{DG}}\right)$, VI - RS method $\left(\boldsymbol{c}_{\mathrm{RS}}\right)$, and clipping procedure $\left(\boldsymbol{c}_{\text {CLIP }}\right)$.

discretization and solution strategy for the steady-state Darcy equations can be found in Appendix B.

6.2. Numerical results. Consider a $50 \mathrm{~m} \times 25 \mathrm{~m}$ rectangular domain with heterogeneous permeability, as shown in Figure 20. The flow will be modeled using Darcy equations, in which the viscosity depends on the concentration of the attendant chemical species, and the transport of the chemical species will be modeled using advection-diffusion equations. For the flow subproblem, we prescribe the pressure boundary conditions on the left and right sides of the domain and no flow boundary conditions on the top and bottom. For the transport subproblem, an initial concentration of unity is prescribed everywhere in the domain, and a Dirichlet boundary condition of zero along the left side and zero flux boundary conditions on the remaining sides are prescribed. A time-step $\Delta t=1$ day is used to simulate the miscible displacement over a time interval $\mathcal{T}=1$ year. All other problem parameters and material properties can be found in Table 10. Figure 21 depicts the concentration profiles under the DG and VI - RS methods at $t=0.5$ and $t=1.0$ years. It can be seen that violations in the maximum principles occur even under the coupled flow and transport computational framework. Furthermore, the violations do not go away as the simulation progresses in time. As it may be difficult to distinguish between the VI - RS and DG or clipping procedures by directly plotting the solutions, we show the differences in the solutions in Figure 22. The ramification shown by the difference plots is that the development of the plumes and 


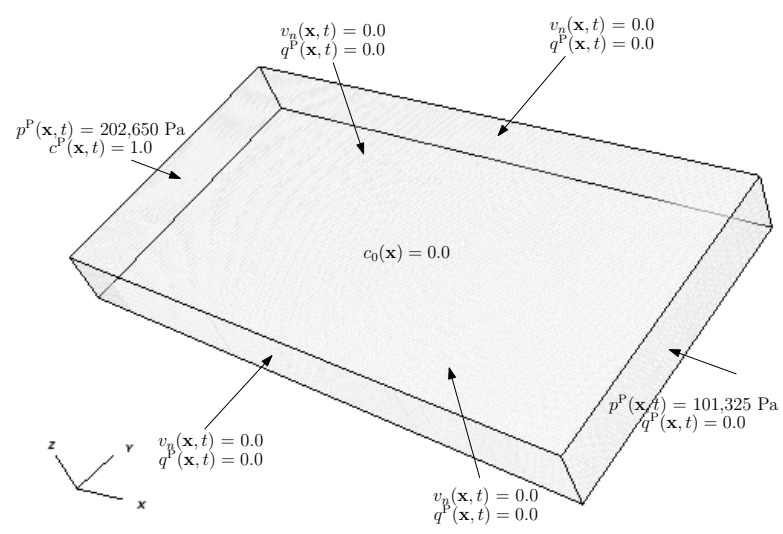

(A) Problem description

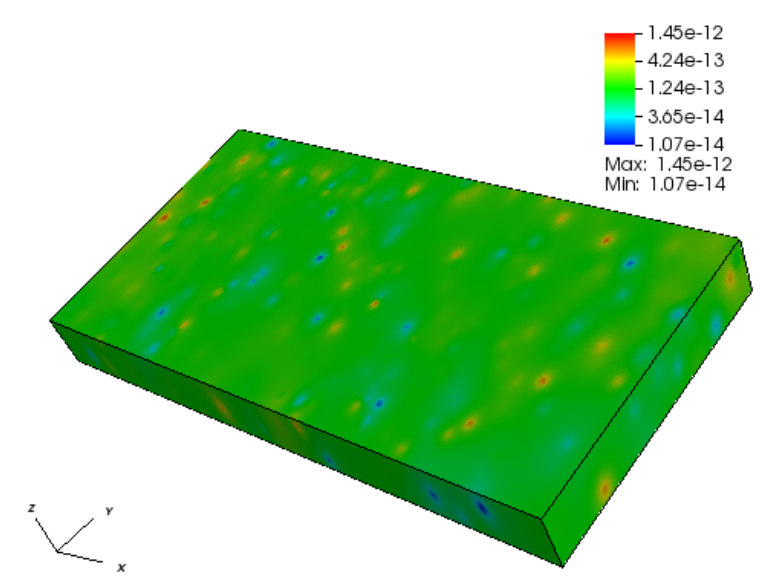

(B) Log scale permeability field $\left(\mathrm{m}^{2}\right)$

Figure 23. 3D miscible displacement: Pictorial description of the boundary value problems $(50 \mathrm{~m} \times 25 \mathrm{~m} \times 5 \mathrm{~m}$ domain with 781,250 structured hexahedrons $)$ for the coupled Darcy and advection-diffusion equations and the corresponding random permeability.

fingerings throughout the computational domain is altered by the VI approach. It is also shown that the clipping procedure is significantly different from the VI approach.

To understand the performance of our VI-based solvers for large-scale versions of this problem, we now consider a $50 \mathrm{~m} \times 25 \mathrm{~m} \times 5 \mathrm{~m}$ box domain with heterogeneous permeability as shown in Figure 23. Table 11 lists all the necessary problem parameters, and the same time-step and time interval from the previous problem is used. This problem is now solved in parallel across 40 MPI processes with a relative residual convergence criterion of $10^{-3}$, and Figure 24 depicts the numerical results

TABLE 11. 3D miscible displacement: problem parameters

\begin{tabular}{ll}
\hline Parameter & Value \\
\hline $\mathbf{b}(\boldsymbol{x})$ & $\{0,0,-9.81\} \mathrm{m} / \mathrm{s}^{2}$ \\
$\mu(c(\mathbf{x}, t))$ & See equation $(6.2 \mathrm{~b})$ \\
$\mu_{0}$ & $3.95 \cdot 10^{-5} \mathrm{~Pa} \mathrm{~s}$ \\
$R_{c}$ & 3 \\
$k(\mathbf{x})$ & varies \\
$f(\mathbf{x}, t)$ & 0 \\
$\rho$ & $479 \mathrm{~kg} / \mathrm{m}^{3}$ \\
$\alpha_{L}$ & $10^{-1} \mathrm{~m}$ \\
$\alpha_{T}$ & $10^{-5} \mathrm{~m}$ \\
$\alpha_{D}$ & $10^{-9} \mathrm{~m}^{2} / \mathrm{s}$ \\
Number of elements & 781,250 \\
Darcy degrees-of-freedom & $3,165,625$ \\
Advection-diffusion degrees-of-freedom & $6,250,000$ \\
\hline
\end{tabular}




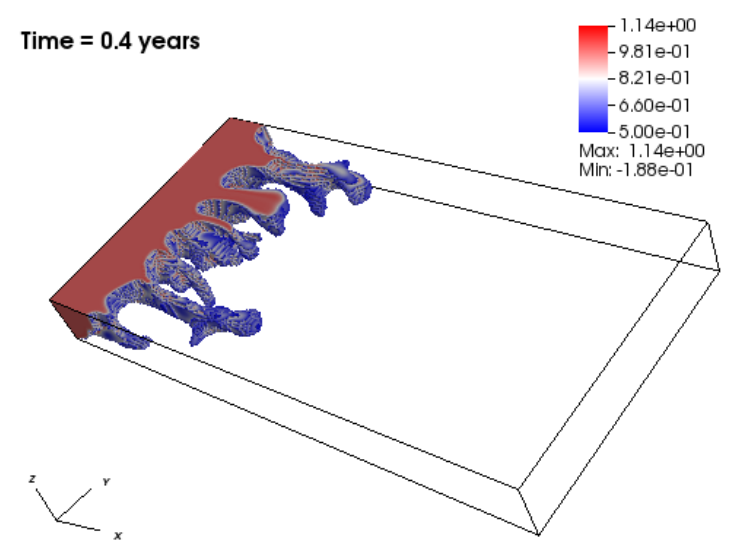

(A) $\boldsymbol{c}_{\mathrm{DG}}$ at $t=0.4$ years

\section{Time $=0.4$ years}

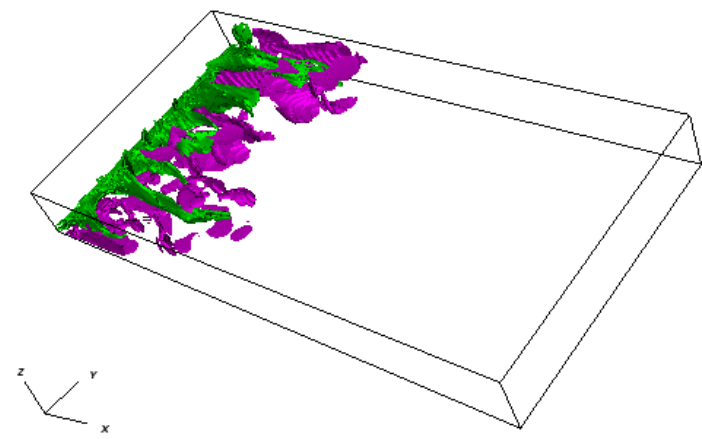

(C) $\boldsymbol{c}_{\mathrm{DG}}$ at $t=0.4$ years

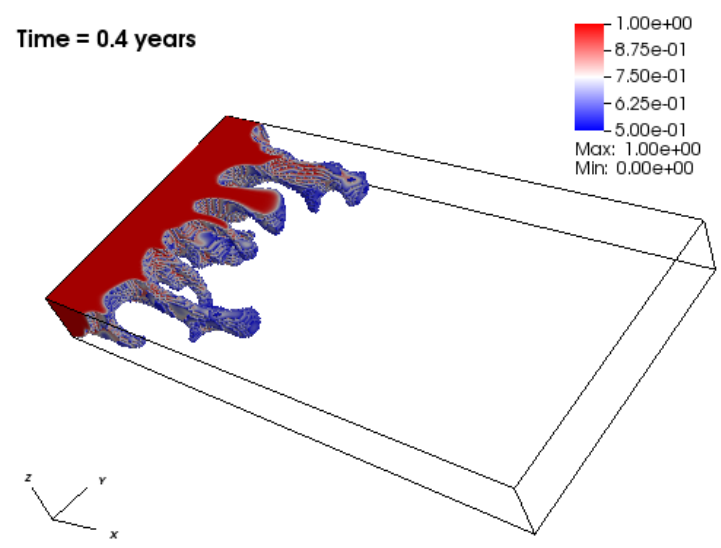

(E) $\boldsymbol{c}_{\mathrm{RS}}$ at $t=0.4$ years

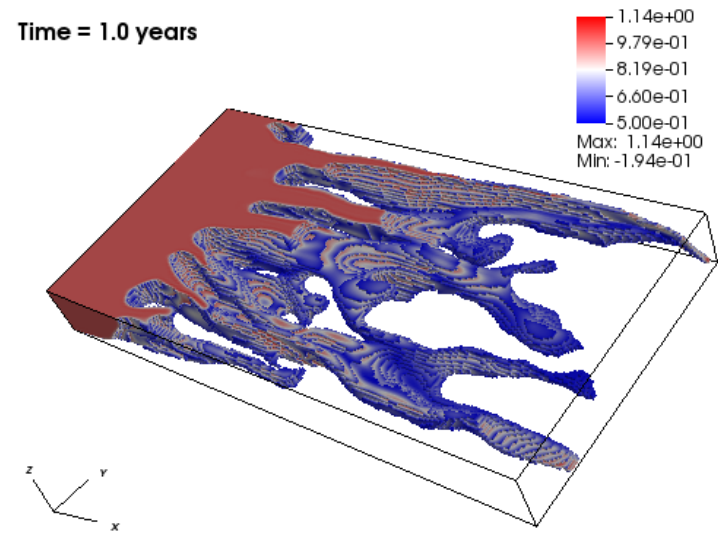

(B) $\boldsymbol{c}_{\mathrm{DG}}$ at $t=1.0$ years

Time $=1.0$ years

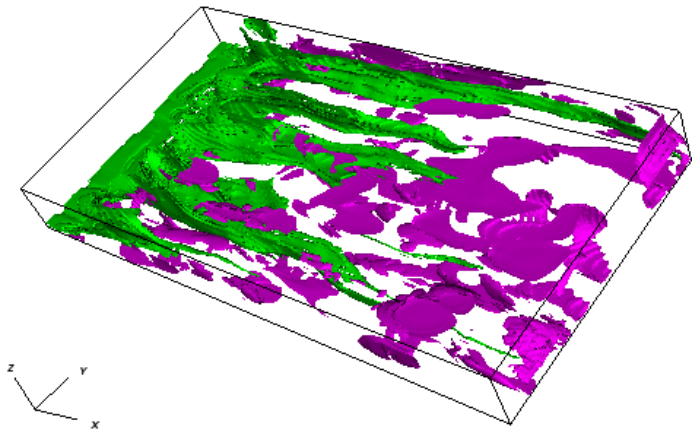

(D) $\boldsymbol{c}_{\mathrm{DG}}$ at $t=1.0$ years

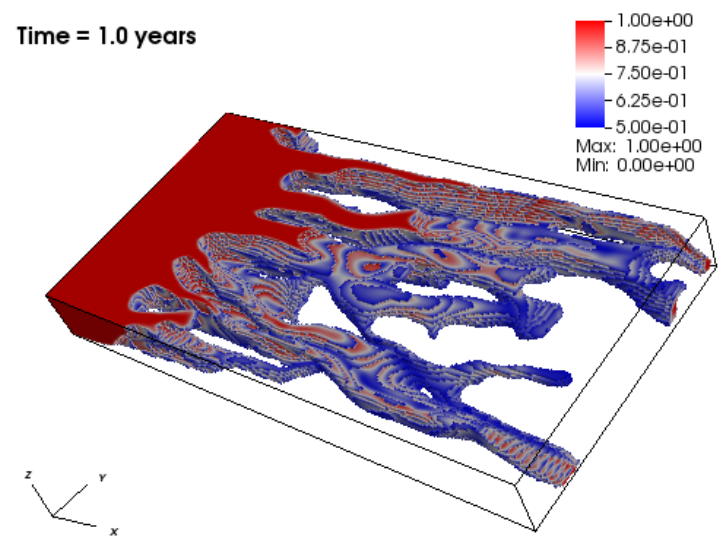

(F) $\boldsymbol{c}_{\mathrm{RS}}$ at $t=1.0$ years

Figure 24. 3D miscible displacement: Top (DG) and bottom (VI) show regions with concentrations above 0.5. Middle figures show regions with concentrations above 1.0 (green) and below 0.0 (purple) (see online version for color figures.) 


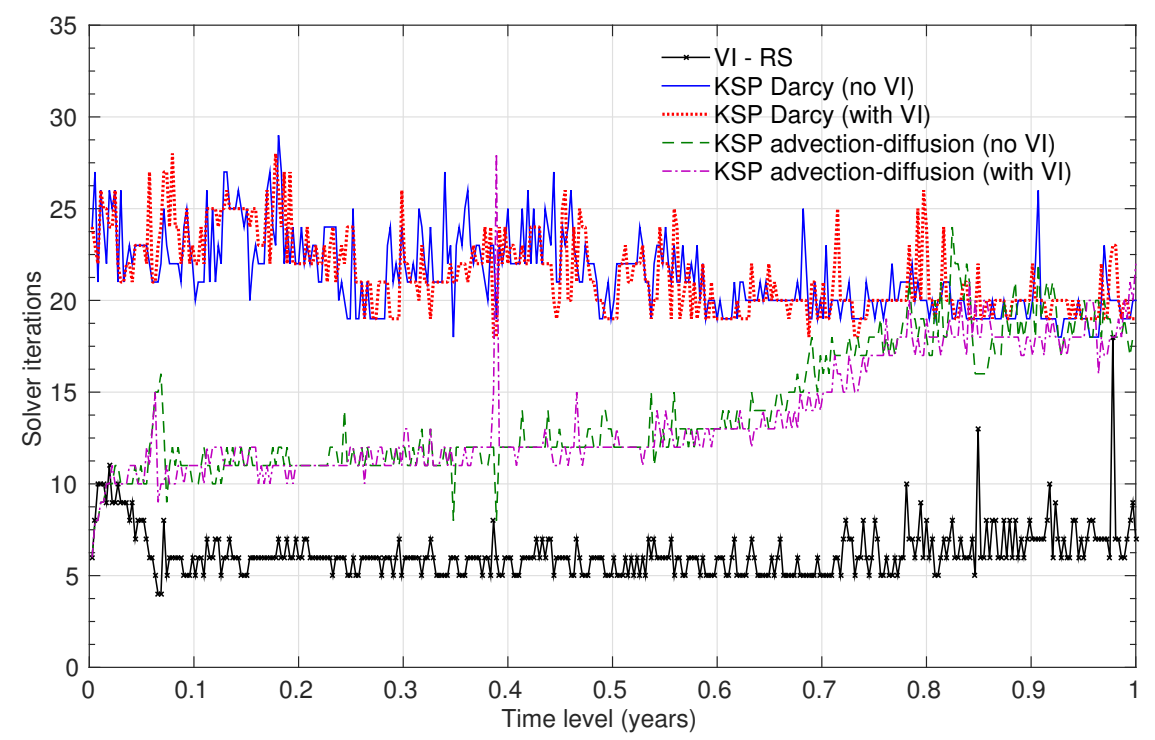

FiguRE 25. 3D miscible displacement: Total number of KSP iterations at each time level for the Darcy and advection-diffusion equation with and without VI. Also shown is the total number of VI iterations at each time level.

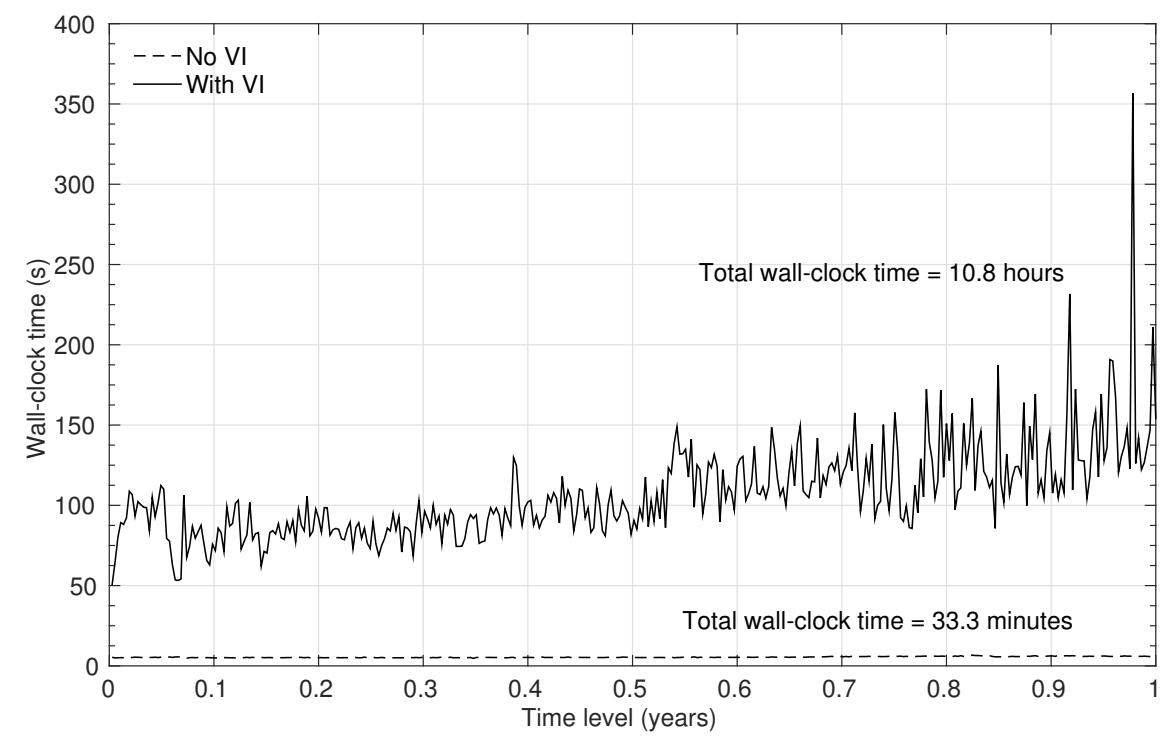

FIGURE 26. 3D miscible displacement: The wall-clock time at each time level with and without VI across 40 cores. Also shown is the sum of the wall-clock time across all time levels.

under the DG formulation without VI - RS and DG formulation with VI - RS. The exact regions where violations in the maximum principle and the non-negative constraint occur are shown in 
Figures $24 \mathrm{c}$ and $24 \mathrm{~d}$. First, we note that this proposed framework can successfully eliminate the violations that occur in a large-scale miscible displacement simulation. We also note that the development and displacement of the plumes is significantly affected by whether VI - RS is applied or not; the differences between Figures $24 \mathrm{~b}$ and $24 \mathrm{f}$ are quite evident unlike the $2 \mathrm{D}$ example. Next, we note from Figure 25 that enforcing the bounded constraints under the VI - RS method will not drastically increase the total number of KSP iterations needed for either the Darcy or advectiondiffusion equations. However, the wall-clock time shown in Figure 26 indicates that the VI - RS method is very expensive. The oscillatory behavior of both the solver iterations and wall-clock time at each time level is largely attributed to the heterogeneous nature of the problem as well as the number of maximum principle violating degrees-of-freedom that naturally arise out of the DG formulation. Although VI-based solvers like VI - RS can enforce maximum principles and the non-negative constraint, we have observed that applying such methodologies can make the overall advection-diffusion finite element simulation up to 20 times as expensive even in a parallel environment.

Before we draw any further conclusions of this paper, we acknowledge that we did not perform a numerical $h$-convergence study. This is due to, despite our best efforts, failure to find an advection-diffusion boundary value problem that considers anisotropy, has an analytical solution, and violates discrete maximum principles. We, therefore, illustrated the performance of the proposed computational framework through other means, as presented in the previous sections.

\section{CONCLUDING REMARKS}

We presented a robust computational framework based on VIs for diffusion and advectiondiffusion equations that satisfies the discrete maximum principles and the non-negative constraint. The framework is applicable to large-scale and transient problems, and can be solved in a parallel setting. The main contributions of this paper and the salient features of the proposed formulation can be summarized as follows.

(C1) Realizing and posing the advection-diffusion problem as a variational inequality (VI) to meet the discrete maximum principles and the non-negative constraint.

(C2) For large-scale problems, we have demonstrated that QP solvers, which is a special case of VIs, are just as good as VI solvers for symmetric and positive-definite problems like the diffusion equation. On the other hand, the proposed VI-based framework can also handle non-self-adjoint operators.

(C3) Unlike the non-negative framework proposed in [Mudunuru and Nakshatrala, 2016a], which is based on a mixed least-squares WF, the proposed framework can utilize any finite element formulation including single-field formulations, and these formulations need not result in symmetric and positive definite coefficient matrices.

(C4) The proposed framework allows one to leverage on existing state-of-the-art computational frameworks for solving VIs. In particular, the Firedrake project, which provides access to parallel solvers in PETSc and TAO libraries, can serve as a suitable platform for implementing the proposed framework, as illustrated in this paper.

(C5) This framework is suitable for many important applications like miscible displacement, subsurface remediation, and transport of radionuclides. In these applications, one encounters not only highly anisotropic medium properties but also highly non-linear phenomena due to aqueous complexation and kinetic reactions. 
A logical next-step of our work is to extend this computational framework to advective-diffusivereactive equations with multiple chemical species along with kinetic reactions.

\section{ACKNOWLEDGMENTS}

The authors acknowledge the financial support from the Department of Energy Office of Science Graduate Student Research (SCGSR) award. The authors also acknowledge the use of the Opuntia Cluster from the Center of Advanced Computing and Data Systems (CACDS) at the University of Houston to carry out the research presented here. The opinions expressed in this paper are those of the authors and do not necessarily reflect those of the sponsors.

\section{Appendix A. Firedrake Project}

The Firedrake project [Rathgeber et al., 2016; Luporini et al., 2016, 2015] is a python-based library that provides an automated system for the solution of partial differential equations using the finite element method. Like the FEniCS Project [Logg et al., 2012; Alnæs et al., 2015], it is also built upon several scientific packages and can employ parallel computing tools across either CPUs or GPUs to obtain the solution. Two of its main leveraged components are the Unified Form Language (UFL) [Alnæs et al., 2014], used to declare finite element discretizations of variational forms, and the PyOP2 system [Rathgeber et al., 2012; Markall et al., 2013], used for the parallel assembly of the finite element discrete formulations. The main difference between the FEniCS and Firedrake project is that all data structures, linear solvers, non-linear solvers, and optimization solvers for the latter are provided entirely by the PETSc and TAO libraries. The mesh can either be generated internally or imported from third party mesh generators like GMSH [Geuzaine and Remacle, 2009], and the parallel partitioning of the mesh is achieved through packages like Chaco [Hendrickson and Leland, 1995]. Another important feature utilized in this paper is extruded meshes. The internal mesh algorithm generates and partitions a $2 \mathrm{D}$ quadrilateral base mesh and is extruded into a hexahedron mesh using the algorithms listed in [Homolya and Ham, 2016; McRae et al., 2016]. To facilitate the readers to be able to reproduce the results presented in this paper, we provided some useful Firedrake-related files below.

Listing 1. 2D GAL diffusion example

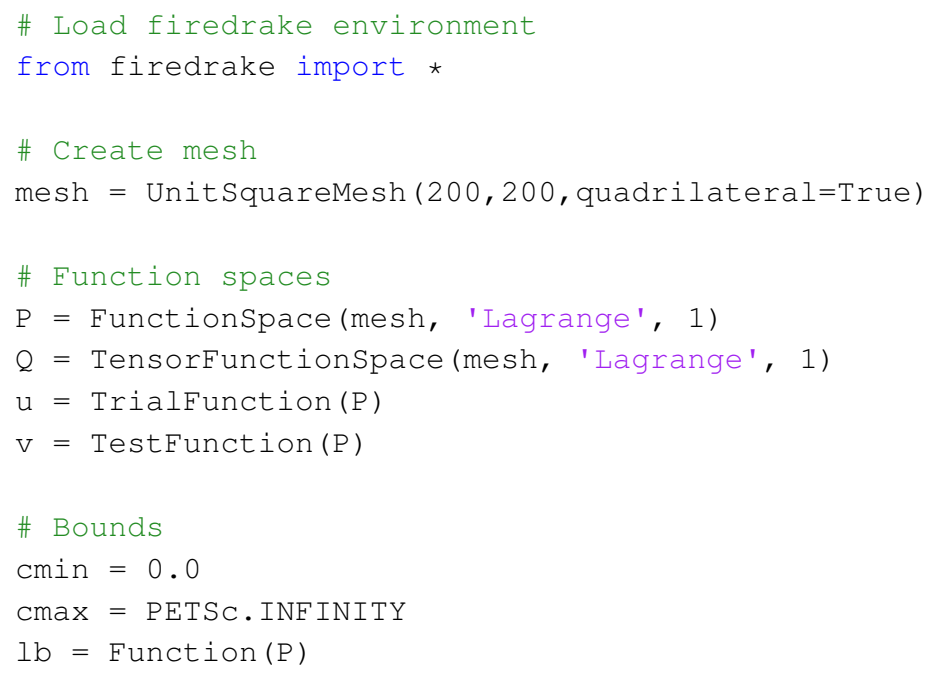




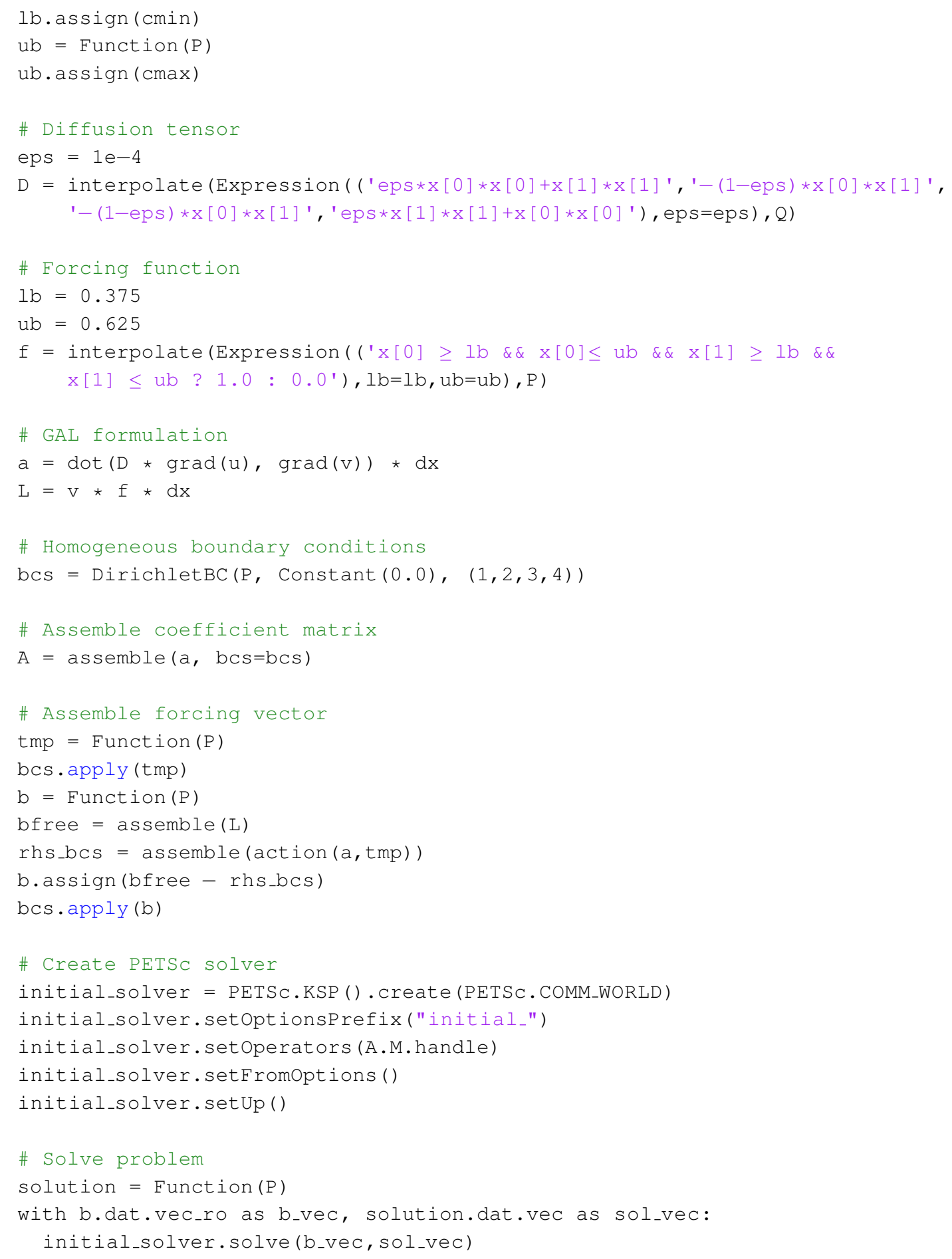

Listing 2. 2D SUPG advection-diffusion example

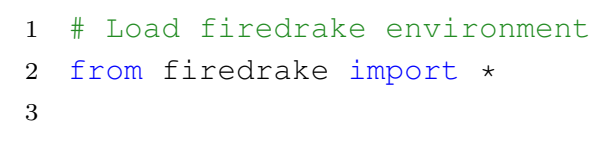




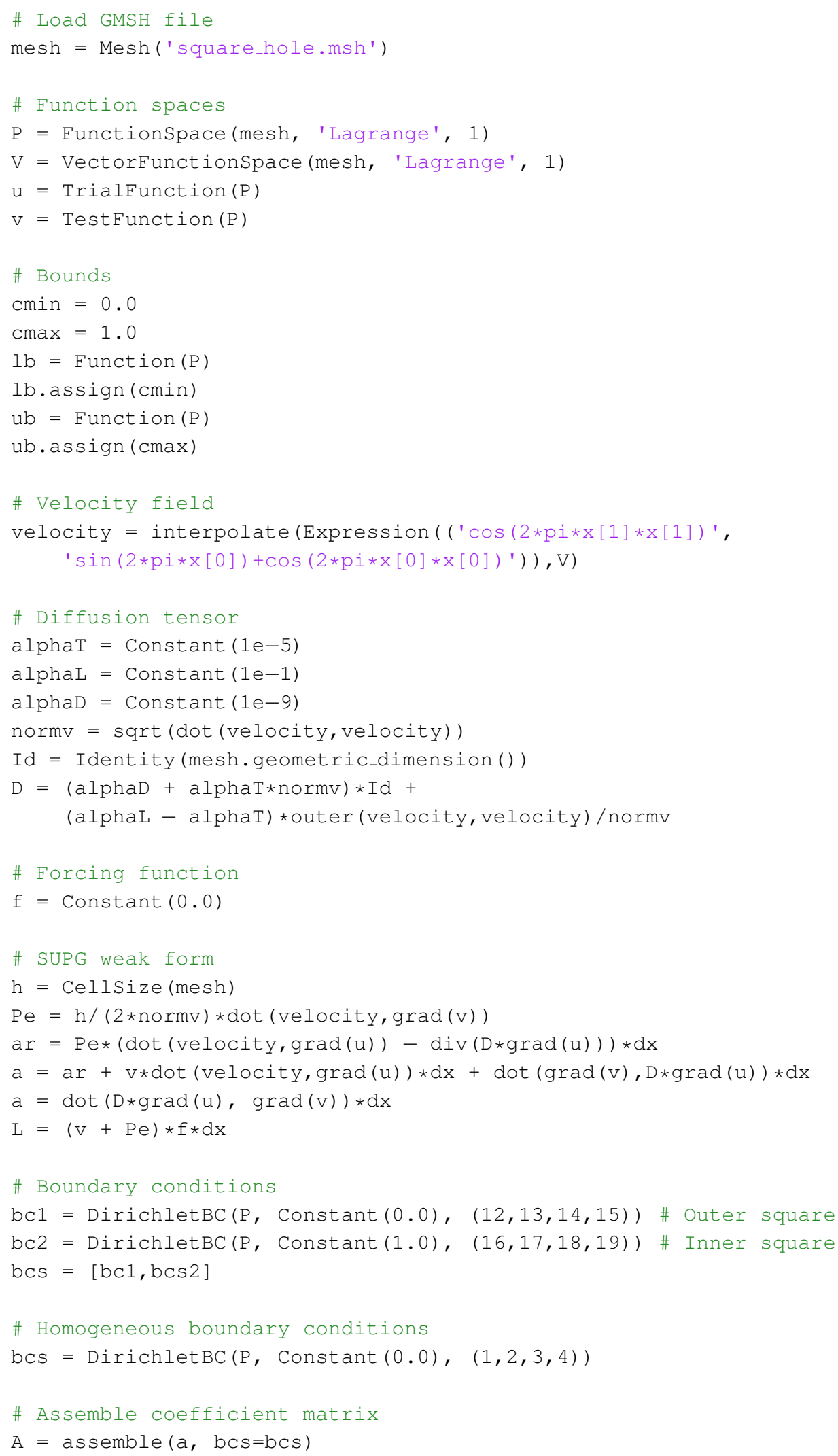




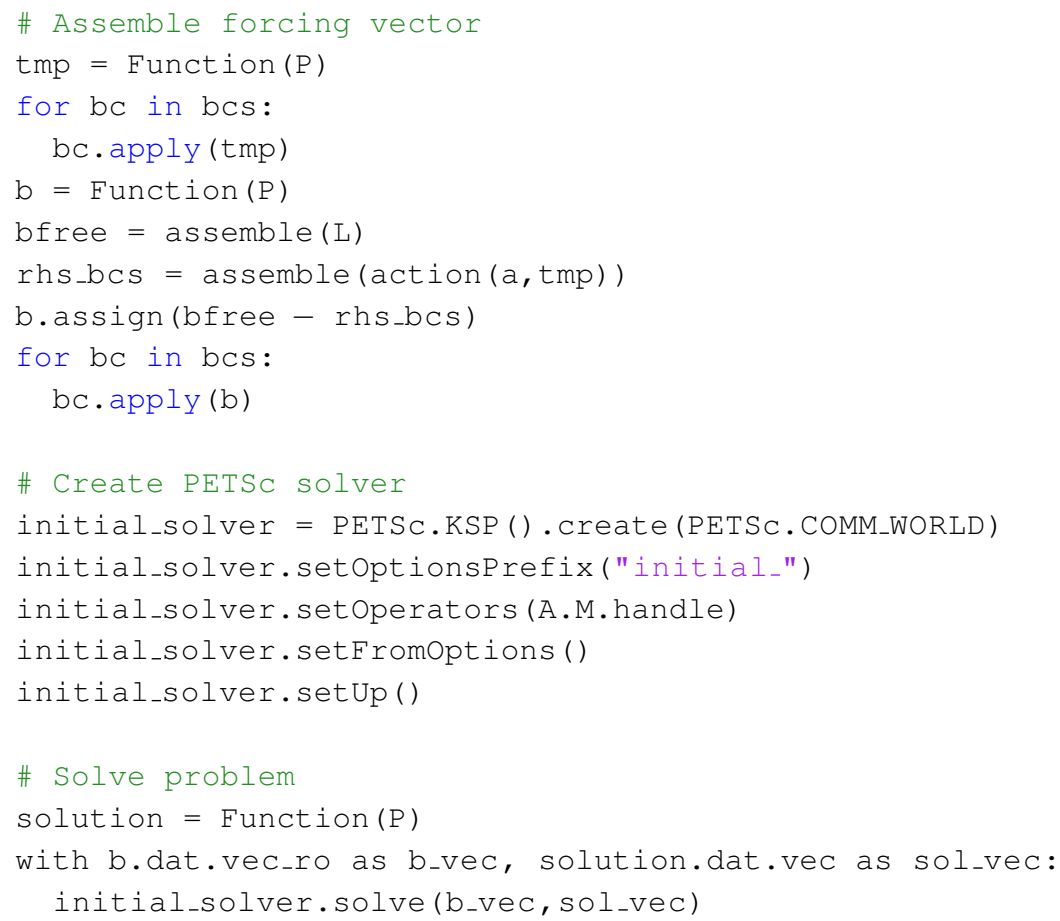

LisTING 3. 3D DG advection-diffusion example

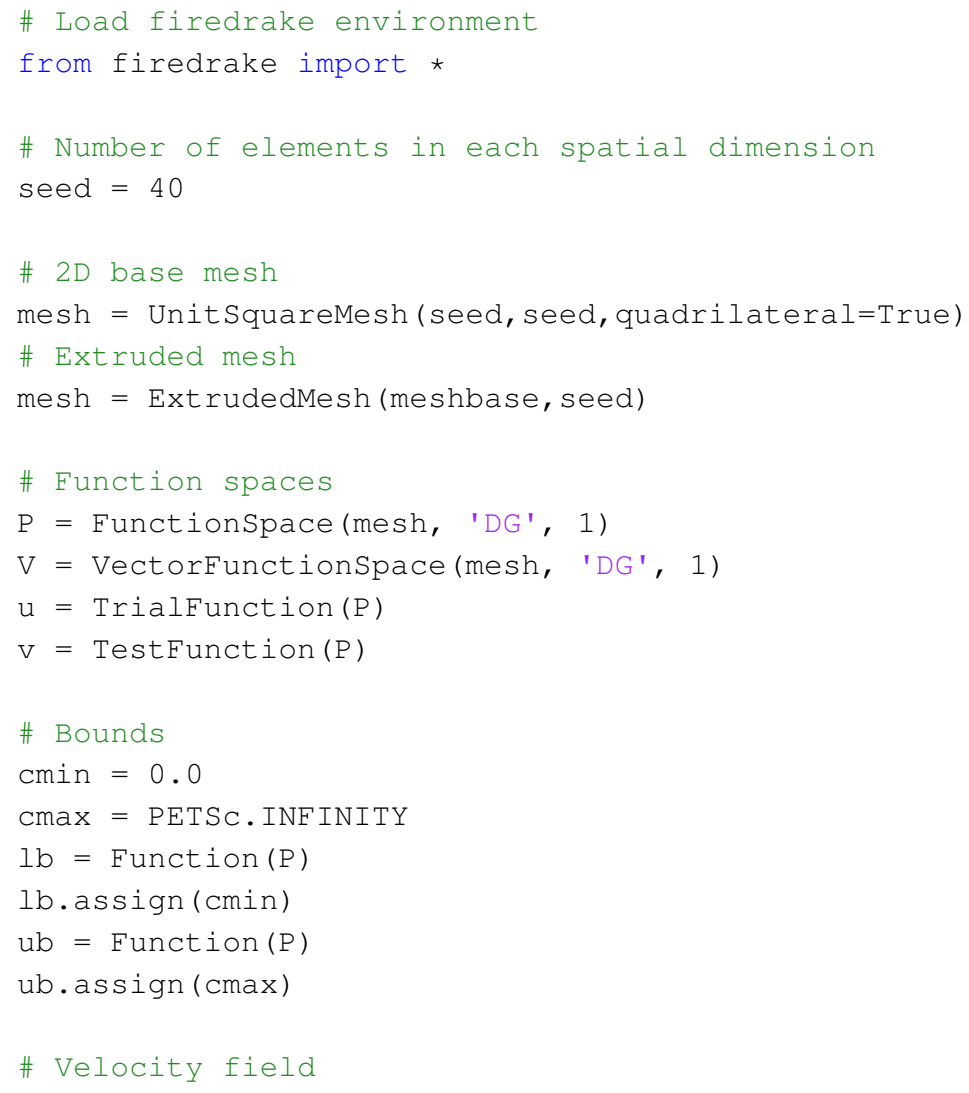




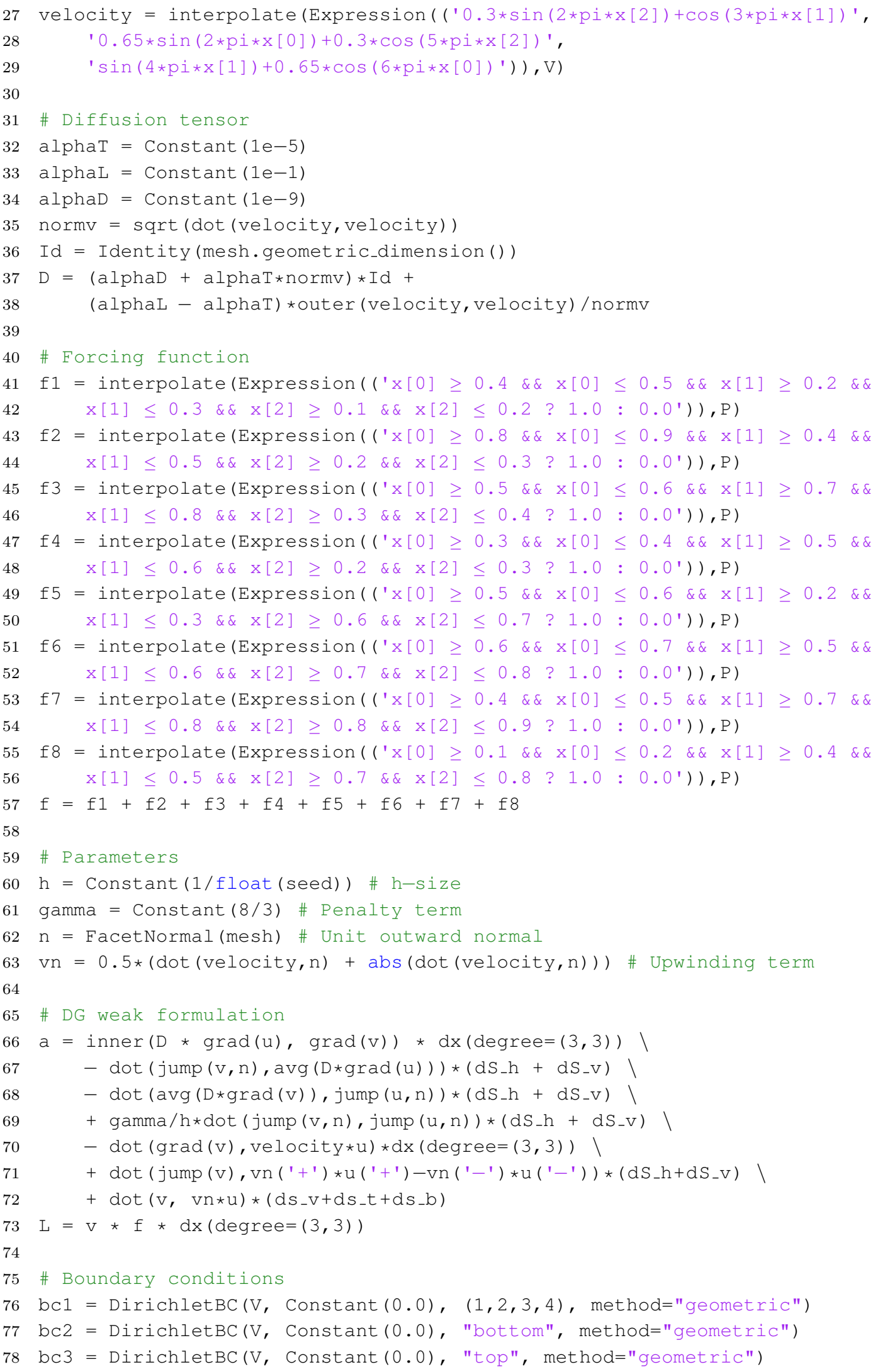




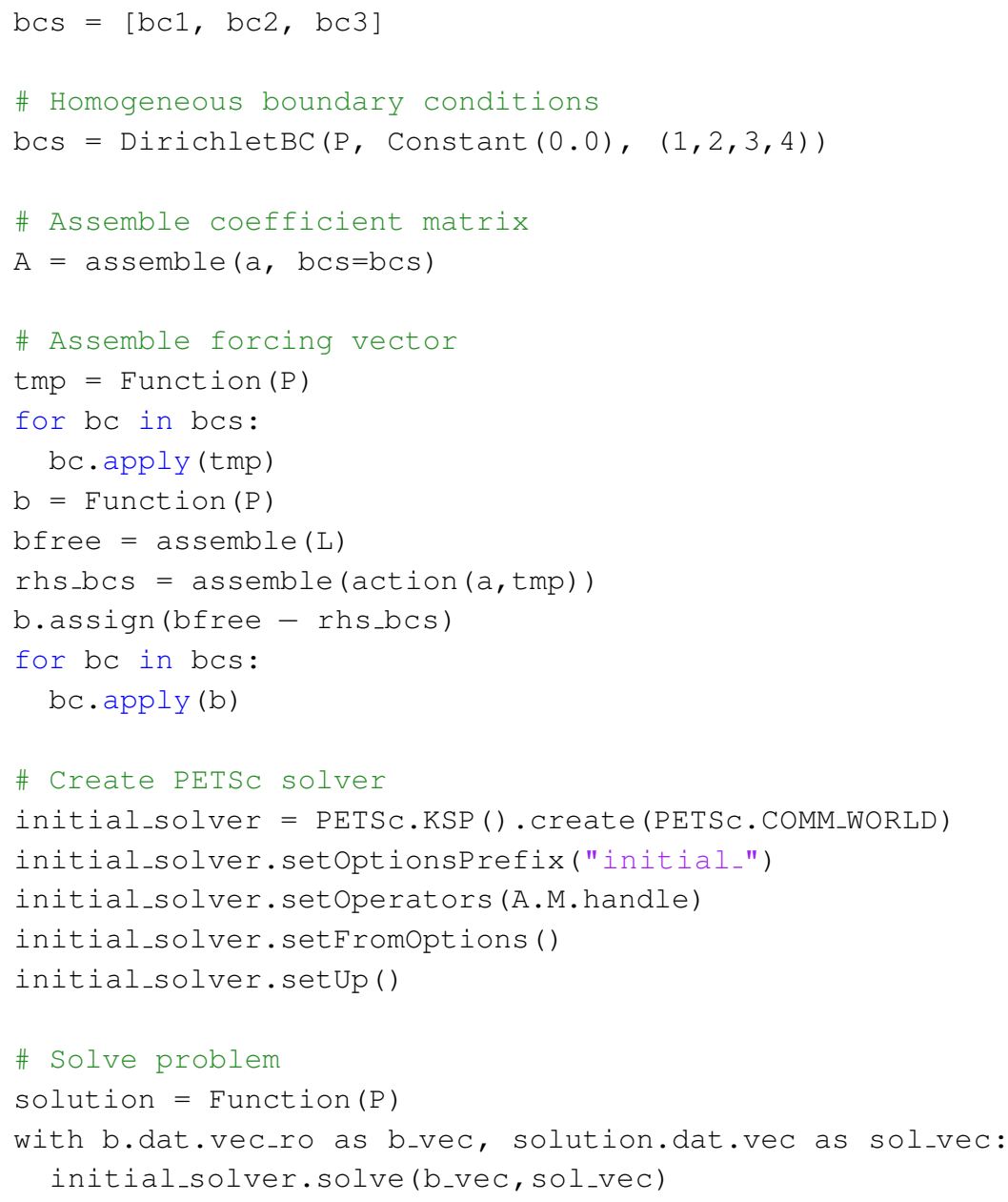

LisTing 4. GMSH geometry file for Listing 2

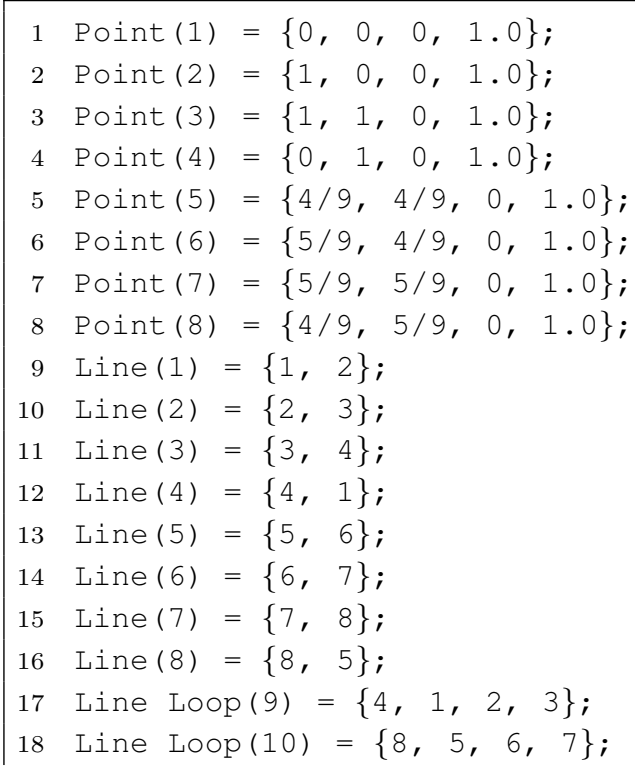




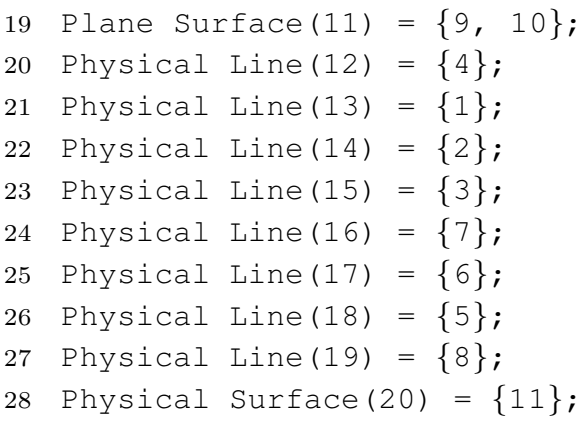

LisTing 5. Semi-smooth (VI - SS) method

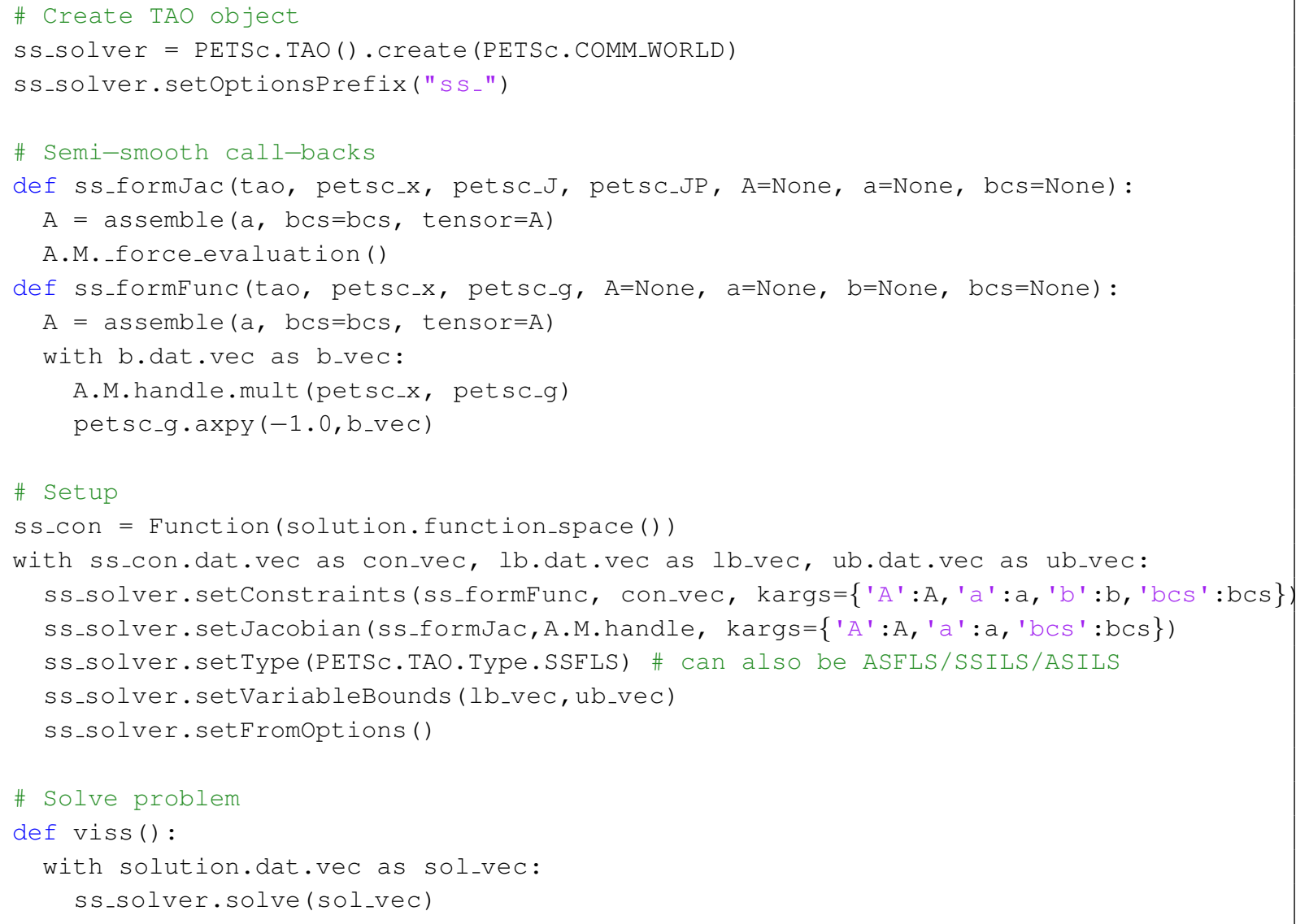

LISTING 6. Reduced-space active set (VI - RS) method

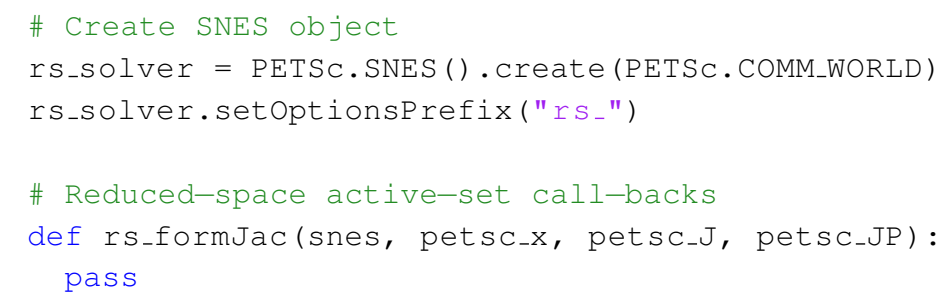




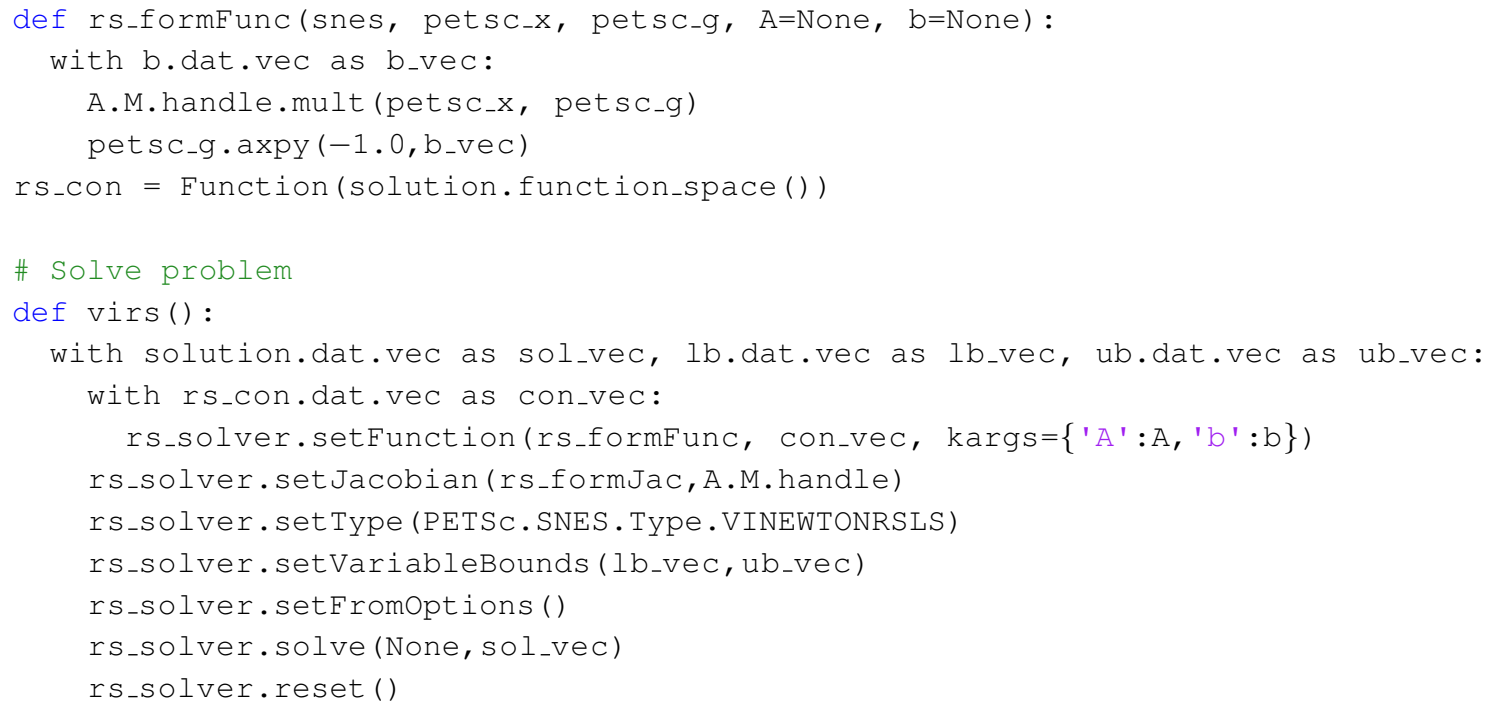

Listing 7. Trust region Newton (QP - TRON) method

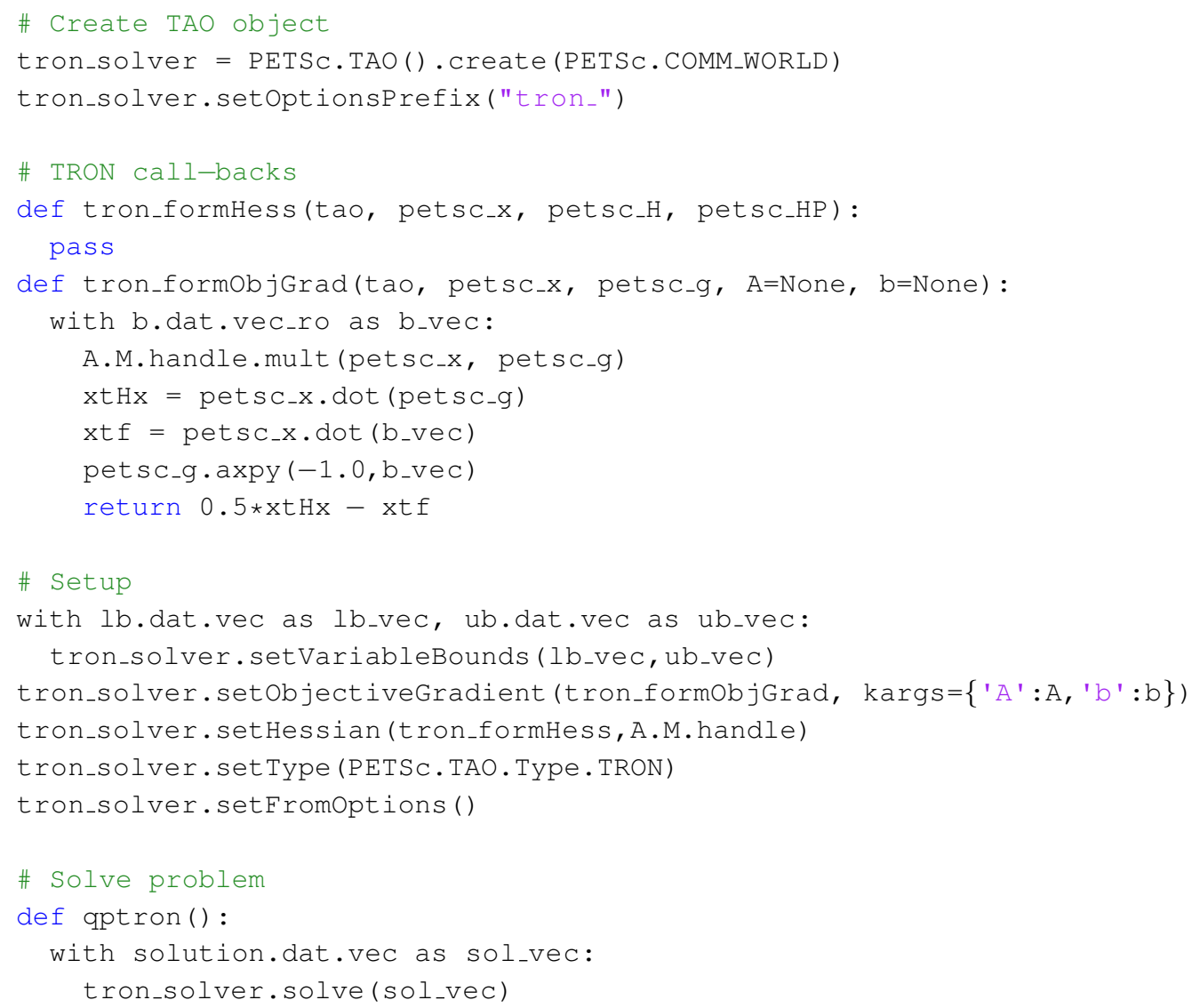




\section{Appendix B. Solution strategy for the Darcy equation}

B.1. Weak formulation. Let $\mathbf{w}(\mathbf{x})$ and $q(\mathbf{x})$ represent the weighting functions for velocity and pressure respectively. The relevant function spaces read as follows:

$$
\begin{aligned}
& \mathcal{V}:=\left\{\mathbf{v} \in\left(L_{2}(\Omega)\right)^{d} \mid \operatorname{div}[\mathbf{v}] \in L_{2}(\Omega), \mathbf{v} \cdot \widehat{\mathbf{n}}=v_{n} \text { on } \Gamma^{v}\right\} \\
& \mathcal{W}:=\left\{\mathbf{w} \in\left(L_{2}(\Omega)\right)^{d} \mid \operatorname{div}[\mathbf{w}] \in L_{2}(\Omega), \mathbf{w} \cdot \widehat{\mathbf{n}}=0 \text { on } \Gamma^{v}\right\} \\
& \mathcal{P}:=L_{2}(\Omega)
\end{aligned}
$$

where $L_{2}(\Omega)$ is the space of square integrable functions. The WF under the classical mixed formulation for the Darcy equations (6.1a) through (6.1d) reads: Find $\mathbf{v}(\mathbf{x}) \in \mathcal{V}$ and $p(\mathbf{x}) \in \mathcal{P}$ such that we have:

$$
\mathcal{B}(\mathbf{w}, q ; \mathbf{v}, p)=\mathcal{L}(\mathbf{w}, q) \quad \forall \mathbf{w}(\mathbf{x}) \in \mathcal{W}, q(\mathbf{x}) \in \mathcal{P}
$$

where the bilinear form and linear functional are:

$$
\begin{aligned}
\mathcal{B}(\mathbf{w}, q ; \mathbf{v}, p) & :=\left(\mathbf{w}(\mathbf{x}) ; \frac{\mu(c(\mathbf{x}))}{k(\mathbf{x})} \mathbf{v}(\mathbf{x})\right)_{\Omega}-(\operatorname{div}[\mathbf{w}(\mathbf{x})] ; p(\mathbf{x}))_{\Omega}-(q(\mathbf{x}) ; \operatorname{div}[\mathbf{w}(\mathbf{x})])_{\Omega} \\
\mathcal{L}(\mathbf{w}, q) & :=(\mathbf{w}(\mathbf{x}) ; \rho \mathbf{b}(\mathbf{x}))_{\Omega}-\left(\mathbf{w}(\mathbf{x}) \cdot \widehat{\mathbf{n}}(\mathbf{x}) ; p^{\mathrm{P}}\right)_{\Gamma^{p}}
\end{aligned}
$$

The lowest order Raviart-Thomas (RT0) space [Raviart and Thomas, 1977] is employed because it ensures element-wise mass conservation. To map the RT0 element onto quadrilateral and extruded hexahedrons, contravariant Piola mapping is used (see [Rognes et al., 2009; Bercea et al., 2016] for further details). The discrete formulations may be assembled into the following block format:

$$
\left(\begin{array}{ll}
\boldsymbol{K}_{v v} & \boldsymbol{K}_{v p} \\
\boldsymbol{K}_{p v} & \boldsymbol{K}_{p p}
\end{array}\right)\left(\begin{array}{l}
\boldsymbol{v} \\
\boldsymbol{p}
\end{array}\right)=\left(\begin{array}{l}
\boldsymbol{f}_{v} \\
\boldsymbol{f}_{p}
\end{array}\right)
$$

where the terms in equation (B.3) respectively correspond to $\boldsymbol{K}_{v v}, \boldsymbol{K}_{v p}$, and $\boldsymbol{K}_{p v}$, and equation (B.4) corresponds to $\boldsymbol{f}_{v}$. It should be noted that $\boldsymbol{K}_{p p}$ and $\boldsymbol{f}_{p}$ are a zero matrix and zero vector, respectively.

B.2. Preconditioning methodology. Equation (B.5) is a saddle-point system which is tricky to precondition effectively for large-scale problems. Several classes of iterative solvers and preconditioning strategies exist for these types of problems [Benzi et al., 2005; Elman et al., 2006; Murphy et al., 2000]. One could alternatively employ hybridization techniques [Cockburn et al., 2009] which introduces Lagrange multipliers to reduce the difficulty of solving such problems. In this paper, we employed a Schur complement approach to precondition the saddle-point system. Conceptually, the problem at hand is a $2 \times 2$ block matrix:

$$
\boldsymbol{K}=\left(\begin{array}{cc}
\boldsymbol{K}_{v v} & \boldsymbol{K}_{v p} \\
\boldsymbol{K}_{p v} & \mathbf{0}
\end{array}\right)
$$

which admits a full factorization of

$$
\boldsymbol{K}=\left(\begin{array}{cc}
\boldsymbol{I} & \mathbf{0} \\
\boldsymbol{K}_{p v} \boldsymbol{K}_{v v}^{-1} & \boldsymbol{I}
\end{array}\right)\left(\begin{array}{cc}
\boldsymbol{K}_{v v} & \mathbf{0} \\
\mathbf{0} & \boldsymbol{S}
\end{array}\right)\left(\begin{array}{cc}
\boldsymbol{I} & \boldsymbol{K}_{v v}^{-1} \boldsymbol{K}_{v p} \\
\mathbf{0} & \boldsymbol{I}
\end{array}\right)
$$

where $\boldsymbol{I}$ is the identity matrix and

$$
\boldsymbol{S}=-\boldsymbol{K}_{p v} \boldsymbol{K}_{v v}^{-1} \boldsymbol{K}_{v p}
$$


is the Schur complement. The inverse can therefore be written as:

$$
\boldsymbol{K}^{-1}=\left(\begin{array}{cc}
\boldsymbol{I} & -\boldsymbol{K}_{v v}^{-1} \boldsymbol{K}_{v p} \\
\mathbf{0} & \boldsymbol{I}
\end{array}\right)\left(\begin{array}{cc}
\boldsymbol{K}_{v v}^{-1} & \mathbf{0} \\
\mathbf{0} & \boldsymbol{S}^{-1}
\end{array}\right)\left(\begin{array}{cc}
\boldsymbol{I} & \mathbf{0} \\
-\boldsymbol{K}_{p v} \boldsymbol{K}_{v v}^{-1} & \boldsymbol{I}
\end{array}\right)
$$

The task at hand is to approximate $\boldsymbol{K}_{v v}^{-1}$ and $\boldsymbol{S}^{-1}$. Since $\boldsymbol{K}_{v v}$ is a mass matrix for the Darcy equation, we can invert it using the ILU(0) (incomplete lower upper) solver. We note that the Schur complement is spectrally a Laplacian, so we can employ a diagonal mass-lumping of $\boldsymbol{K}_{v v}$ to give a good approximation to $\boldsymbol{K}_{v v}^{-1}$. That is, we can use

$$
\boldsymbol{S}_{p}=-\boldsymbol{K}_{p v} \operatorname{diag}\left(\boldsymbol{K}_{v v}\right)^{-1} \boldsymbol{K}_{v p}
$$

to precondition the inner solver inverting $\boldsymbol{S}$. For this block we employ the multi-grid V-cycle on $\boldsymbol{S}_{p}$ using the Trilinos ML package ([Sala et al., 2004]). These blocks are symmetric and positivedefinite so one could employ the CG solvers to obtain the inverses. When the inverses are obtained, only a single sweep of flexible GMRES is needed to obtain the full solution. However, instead of individually solving for $\boldsymbol{K}_{v v}^{-1}$ and $\boldsymbol{S}_{p}$, we could alternatively apply a single sweep of ILU(0) and multi-grid, respectively, and rely on GMRES to solve the entire block system. By providing less accurate approximations of the inner individual blocks, the number of GMRES iterations for the overall system increases but the numerical accuracy remains the same. We have found that this methodology is computationally less expensive and more practical for large-scale computations. One could alternatively employ one of the factorizations (either lower or upper) to decrease the computational cost associated with setting up the preconditioner. Below are some necessary PETSc command-line options for the described Schur complement approach.

Listing 8. PETSc solver options for the Schur complement approach

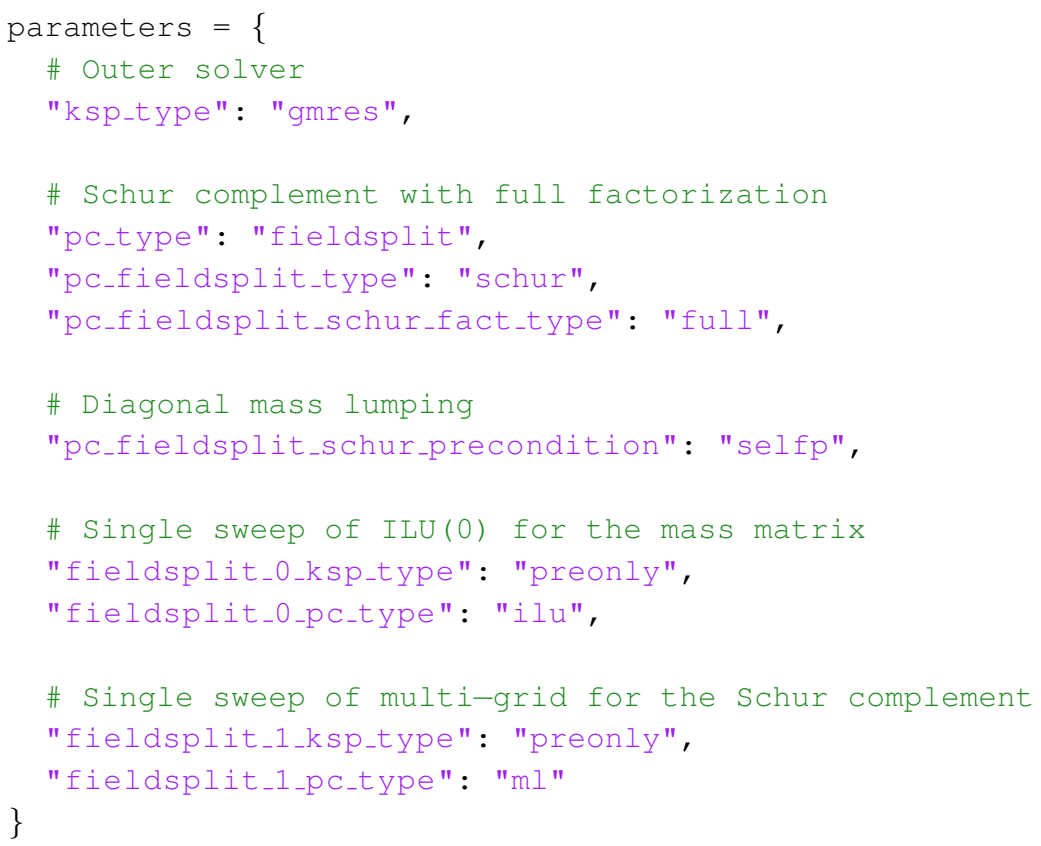




\section{References}

M. Alnæs, J. Blechta, J. Hake, A. Johansson, B. Kehlet, A. Logg, C. Richardson, J. Ring, M. E. Rognes, and G. N. Wells. The fenics project version 1.5. Archive of Numerical Software, 3(100): 9-23, 2015.

M. S. Alnæs, A. Logg, K. B. Ølgaard, M. E. Rognes, and G. N. Wells. Unified form language: A domain-specific language for weak formulations of partial differential equations. ACM Transactions on Mathematical Software, 40(2):9, 2014.

L. Armijo. Minimization of functions having Lipschitz-continuous first partial derivatives. Pacific Journal of Mathematics, 16:1-3, 1966.

D. N. Arnold, F. Brezzi, B. Cockburn, and L. D. Marini. Unified analysis of discontinuous Galerkin methods for elliptic problems. SIAM Journal on Numerical Analysis, 39:1749-1779, 2002.

S. Balay, W. D. Gropp, L. C. McInnes, and B. F. Smith. Efficient management of parallelism in object oriented numerical software libraries. In E. Arge, A. M. Bruaset, and H. P. Langtangen, editors, Modern Software Tools in Scientific Computing, pages 163-202. Birkhäuser Press, 1997.

S. Balay, S. Abhyankar, M. F. Adams, J. Brown, P. Brune, K. Buschelman, L. Dalcin, V. Eijkhout, W. D. Gropp, D. Kaushik, M. G. Knepley, L. C. McInnes, K. Rupp, B. F. Smith, S. Zampini, H. Zhang, and H. Zhang. PETSc users manual. Technical Report ANL-95/11 - Revision 3.7, Argonne National Laboratory, 2016.

S. Benson and T. S. Munson. Flexible complementarity solvers for large-scale applications. Pacific Journal of Mathematics, 21:155-168, 2006.

M. Benzi, G. H. Golub, and J. Liesen. Numerical solution of saddle point problems. Acta Numerica, 14:1-137, 2005.

G. T. Bercea, A. T. T. McRae, D. A. Ham, L. Mitchell, F. Rathgeber, L. Nardi, F. Luporini, and P. H. J. Kelly. A structure-exploiting numbering algorithm for finite elements on extruded meshes, and its performance evaluation in firedrake. Geoscientific Model Development, 9:38033815, 2016.

S. Boyd and L. Vandenberghe. Convex Optimization. Cambridge University Press, Cambridge, UK, 2004.

S. C. Brenner and L. R. Scott. The Mathematical Theory of Finite Element Methods. SpringerVerlag, New York, USA, 1994.

H. Brezis. Functional Analysis, Sobolev Spaces and Partial Differential Equations. Springer Science \& Business Media, 2010.

A. N. Brooks and T. J. R. Hughes. Streamline upwind/Petrov-Galerkin formulations for convection dominated flows with particular emphasis on the incompressible Navier-Stokes equations. Computer Methods in Applied Mechanics and Engineering, 32:199-259, 1982.

O. Burdakov, I. Kapyrin, and Y. Vassilevski. Monotonicity recovering and accuracy preserving optimization methods for postprocessing finite element solutions. Journal of Computational Physics, 231:3126-3142, 2012.

J. Chang, K. B. Nakshatrala, and J. N. Reddy. Modification to Darcy-Forchheimer model due to pressure-dependent viscosity: consequences and numerical solutions. To appear in Journal of Porous Media, 2016.

J. Chang, S. Karra, and K. B. Nakshatrala. Large-scale optimization-based non-negative computational framework for diffusion equations: Parallel implementation and performance studies. Journal of Scientific Computing, 70:243-271, 2017. 
C. Y. Chen and E. Meiburg. Miscible porous media displacements in the quarter five-spot configuration. part 1. the homogeneous case. Journal of Fluid Mechanics, 371:233-268, 1998a.

C. Y. Chen and E. Meiburg. Miscible porous media displacements in the quarter five-spot configuration. part 2. effect of heterogeneities. Journal of Fluid Mechanics, 371:269-299, 1998b.

M. Chipot. Elliptic Equations: An Introductory Course. Birkhäuser, Basel, Switzerland, 2009.

P. G. Ciarlet and P. A. Raviart. Maximum principle and uniform convergence for the finite element method. Computer Methods in Applied Methods and Engineering, 2:17-31, 1973.

B. Cockburn. Discontinuous Galerkin methods. Zeitschrift für Angewandte Mathematik und Mechanik, 83(11):731-754, 2003. doi: 10.1002/zamm.200310088.

B. Cockburn, J. Gopalakrishnan, and R. Lazarov. Unified hybridization of discontinuous Galerkin, mixed, and continuous Galerkin methods for second order elliptic problems. SIAM Journal on Numerical Analysis, 47(2):1319-1365, 2009.

L. B. da Veiga, K. Lipnikov, and G. Manzini. The mimetic finite difference method for elliptic problems, volume 11. Springer, 2014.

L. D. Dalcin, R. R. Paz, P. A. Kler, and A. Cosimo. Parallel distributed computing using Python. Advances in Water Resources, 34(9):1124-1139, 2011.

J. W. Demmel. Applied Numerical Linear Algebra. Society for Industrial and Applied Mathematics, Philadelphia, PA, USA, 1997.

T. Dombre, U. Frisch, J. M. Greene, M. Hénon, A. Mehr, and A. M. Soward. Chaotic streamlines in the ABC flows. Journal of Fluid Mechanics, 167:353-391, 1986.

G. Duvaut and J. L. Lions. Inequalities in Mechanics and Physics. Springer-Verlag, Berlin, Germany, 1976.

H. C. Elman, D. J. Silvester, and A. J. Wathen. Finite elements and fast iterative solvers. Journal of Fluid Mechanics, 557(1):474-475, 2006.

US EPA. Cleaning up the nation's waste sites: Markets and technology trends. Technical Report EPA 542-R-04-015, 2004.

F. Facchinei and J.-S. Pang. Finite-Dimensional Variational Inequalities and Complementarity Problems. Volume I. Springer-Verlag, New York, USA, 2003.

G. Fichera. Problemi elastostatici con vincoli unilaterali: il problema di Signorini con ambigue condizioni al contorno. Accademia nazionale dei Lincei, 1964.

G. Fichera. Linear Elliptic Differential Systems and Eigenvalue Problems. Number 8. Springer, 1965.

A. Fischer. A special Newton-type optimization method. Optimization, 24:269-284, 1992.

A. Genty and C. Le Potier. Maximum and minimum principles for radionuclide transport calculations in geological radioactive waste repository: comparison between a mixed hybrid finite element method and finite volume element discretizations. Transport in Porous Media, 88:65-86, 2011.

C. Geuzaine and J. F. Remacle. Gmsh: a three-dimensional finite element mesh generator with built-in pre- and post-processing facilities. International Journal for Numerical Methods in Engineering, 79:1309-1331, 2009.

J. W. Gibbs. Fourier's series. Nature, 59(1522):200, 1898.

J. W. Gibbs. Fourier's series. Nature, 59(1539):606, 1899.

D. Gilbarg and N. S. Trudinger. Elliptic Partial Differential Equations of Second Order. Springer, New York, USA, 2001. 
R. Glowinski. Numerical Methods for Nonlinear Variational Problems. Springer-Verlag, Berlin, Germany, 1984.

C. Grossmann, H. G. Roos, and M. Stynes. Numerical Treatment of Partial Differential Equations. Springer, Berlin, Germany, 2007.

G. E. Hammond and P. C. Lichtner. Field-scale model for the natural attenuation of uranium at the hanford 300 area using high-performance computing. Water Resources Research, 46:W09602, 2010 .

W. Han and B. D. Reddy. Plasticity: Mathematical Theory and Numerical Analysis, volume 9. Springer Science \& Business Media, 2012.

I. Harari. Stability of semidiscrete formulations for parabolic problems at small time steps. Computer Methods in Applied Mechanics and Engineering, 193(15):1491-1516, 2004.

D. R. Harp and V. V. Vesselinov. Contaminant remediation decision analysis using information gap theory. Stochastic Environmental Research and Risk Assessment, 27:159-168, 2013.

J. M. Heikoop, T. M. Johnson, K. H. Birdsell, P. Longmire, D. D. Hickmott, E. P. Jacobs, D. E. Broxton, D. Katzman, V. V. Vesselinov, M. Ding, D. T. Vanimana, S. L. Reneaua, T. J. Goering, J. Glessnerb, and A. Basu. Isotopic evidence for reduction of anthropogenic hexavalent chromium in Los Alamos National Laboratory groundwater. Chemical Geology, 373:1-9, 2014.

B. Hendrickson and R. Leland. A multilevel algorithm for partitioning graphs. In Supercomputing '95: Proceedings of the 1995 ACM/IEEE Conference on Supercomputing (CDROM), page 28, New York, 1995. ACM Press. ISBN 0-89791-816-9. doi: http://doi.acm.org/10.1145/224170. 224228.

I. Hlavacek, J. Haslinger, J. Necas, and J. Lovisek. Solution of Variational Inequalities in Mechanics, volume 66. Springer Science \& Business Media, 2012.

M. Homolya and D. A. Ham. A parallel edge orientation algorithm for quadrilateral meshes. SIAM Journal on Scientific Computing, 38:S48-S61, 2016.

W. Huang and Y. Wang. Discrete maximum principle for the weak Galerkin method for anisotropic diffusion problems. Communications in Computational Physics, 18(1):65-90, 2015.

N. Kikuchi and J. T. Oden. Contact Problems in Elasticity: A Study of Variational Inequalities and Finite Element Methods, volume 8. SIAM, 1988.

D. Kinderlehrer and G. Stampacchia. An Introduction to Variational Inequalities and Their Applications. SIAM Classics in Applied Mathematics, New York, USA, 2000.

HYPRE: High Performance Preconditioners. Lawrence Livermore National Laboratory. http: //www.llnl.gov/CASC/hypre/.

D. R. Lester, G. Metcalfe, and M. G. Trefry. Is chaotic advection inherent to porous media flow? Physical Review Letters, 111(17):174101, 2013.

J. Li and B. Riviére. High order discontinuous Galerkin method for simulating miscible flooding in porous media. Computational Geoscience, 19:1251-1268, $2015 \mathrm{a}$.

J. Li and B. Riviére. Numerical solutions of the incompressible miscible displacement equations in heterogeneous media. Computer Methods in Applied Mechanics and Engineering, 292:107-121, 2015b.

J. Li and B. Riviére. Numerical modeling of miscible viscous fingering instabilities by high-order methods. Transport in Porous Media, 113:607628, 2016.

C. J. Lin and J. Moré. Newton's method for large bound-constrained optimization problems. SIAM Journal on Optimization, 9:1100-1127, 1999. 
J. L. Lions and G. Stampacchia. Variational inequalities. Communications on Pure and Applied Mathematics, 20(3):493-519, 1967.

K. Lipnikov, M. Shashkov, D. Svyatskiy, and Y. Yassilevski. Monotone finite volume schemes for diffusion equations on unstructured triangular and shape-regular polygonal meshes. Journal of Computational Physics, 227:492-512, 2007.

R. Liska and M. Shashkov. Enforcing the discrete maximum principle for linear finite element solutions for elliptic problems. Communications in Computational Physics, 3:852-877, 2008.

A. Logg, K. A. Mardal, and G. N. Wells. Automated solution of differential equations by the finite element method: The FEniCS book, volume 84. Springer Science \& Business Media, 2012.

T. De Luca, F. Facchinei, and C. Kanzow. A semismooth equation approach to the solution of nonlinear complementarity problems. Mathematical Programming, 75:407-439, 1996.

F. Luporini, A. L. Varbanescu, F. Rathgeber, G. T. Bercea, J. Ramanujam, D. A. Ham, and P. H. J. Kelly. Cross-loop optimization of arithmetic intensity for finite element local assembly. ACM Transactions on Architecture and Code Optimization, 11(4):57:1-57:25, 2015.

F. Luporini, D. A. Ham, and P. H. J. Kelly. An algorithm for the optimization of finite element integration loops. Submitted to ACM TOMS, 2016. URL http://arxiv.org/abs/1604.05872.

G. R. Markall, F. Rathgeber, L. Mitchell, N. Loriant, C. Bertolli, D. A. Ham, and P. H. J. Kelly. Performance-portable finite element assembly using pyop2 and fenics. In Julian Martin Kunkel, Thomas Ludwig, and Hans Werner Meuer, editors, 28th International Supercomputing Conference, ISC, Proceedings, volume 7905 of Lecture Notes in Computer Science, pages 279289. Springer, 2013. doi: 10.1007/978-3-642-38750-0_21. URL http://dx.doi.org/10.1007/ 978-3-642-38750-0_21.

A. T. T. McRae, G. T. Bercea, L. Mitchell, D. A. Ham, and C. J. Cotter. Automated generation and symbolic manipulation of tensor product finite elements. SIAM Journal on Scientific Computing, 38:S25-S47, 2016.

R. Mifflin. Semismooth and semiconvex functions in constrained optimization. SIAM Journal on Control and Optimization, 15:957-972, 1977.

M. K. Mudunuru and K. B. Nakshatrala. On enforcing maximum principles and achieving elementwise species balance for advection-diffusion-reaction equations under the finite element method. Journal of Computational Physics, 305:448-493, 2016a.

M. K. Mudunuru and K. B. Nakshatrala. On mesh restrictions to satisfy comparison principles, maximum principles, and the non-negative constraint: Recent developments and new results. Mechanics of Advanced Materials and Structures, DOI: 10.1080/15502287.2016.1166160, 2016b.

M. K. Mudunuru, M. Shabouei, and K. B. Nakshatrala. On local and global species conservation errors for nonlinear ecological models and chemical reacting flows. In ASME 2015 International Mechanical Engineering Congress and Exposition, pages V009T12A018-V009T12A018. American Society of Mechanical Engineers, 2015.

T. Munson, J. Sarich, S. Wild, S. Benson, and L. C. McInnes. Toolkit for advanced optimization (tao) users manual. Technical Report ANL/MCS-TM-322 - Revision 3.5, Argonne National Laboratory, 2014. URL http://www.mcs.anl.gov/tao.

T. S. Munson, F. Facchinei, M. C. Ferris, A. Fischer, and C. Kanzow. The semismooth algorithm for large scale complementarity problems. INFORMS Journal on Computing, 13:294-311, 2001.

M. F. Murphy, G. H. Golub, and A. J. Wathen. A note on preconditioning for indefinite linear systems. SIAM Journal on Scientific Computing, 21(6):1969-1972, 2000. 
H. Nagarajan and K. B. Nakshatrala. Enforcing the non-negativity constraint and maximum principles for diffusion with decay on general computational grids. International Journal for Numerical Methods in Fluids, 67:820-847, 2011.

A. Nagurney. Variational Inequalities. Technical report, Isenberg School of Management, University of Massachusetts Amherst, 2002. URL https://supernet.isenberg.umass.edu/Austria_ Lectures/fvisli.pdf.

A. Nagurney and D. Zhang. Projected Dynamical Systems and Variational Inequalities with Applications, volume 2. Springer Science \& Business Media, 2012.

K. B. Nakshatrala and A. J. Valocchi. Non-negative mixed finite element formulations for a tensorial diffusion equation. Journal of Computational Physics, 228:6726-6752, 2009.

K. B. Nakshatrala, M. K. Mudunuru, and A. J. Valocchi. A numerical framework for diffusioncontrolled bimolecular-reactive systems to enforce maximum principles and the non-negative constraint. Journal of Computational Physics, 253:278-307, 2013.

K. B. Nakshatrala, H. Nagarajan, and M. Shabouei. A numerical methodology for enforcing maximum principles and the non-negative constraint for transient diffusion equations. Communications in Computational Physics, 19:53-93, 2016.

R. K. Pal, R. Abedi, A. Madhukar, and R. B. Haber. Adaptive spacetime discontinuous Galerkin method for hyperbolic advection-diffusion with a non-negativity constraint. International Journal for Numerical Methods in Engineering, 105:963-989, 2016.

G. S. Payette, K. B. Nakshatrala, and J. N. Reddy. On the performance of high-order finite elements with respect to maximum principles and the non-negative constraint for diffusion-type equations. International Journal for Numerical Methods in Engineering, 91:742-771, 2012.

C. Le Potier. Finite volume monotone scheme for highly anisotropic diffusion operators on unstructured triangular meshes. Comptes Rendus Mathematique, 341:787-792, 2005.

Anna Pyzara, Beata Bylina, and Jarosław Bylina. The influence of a matrix condition number on iterative methods' convergence. In Computer Science and Information Systems (FedCSIS), 2011 Federated Conference on, pages 459-464. IEEE, 2011.

L. Qi. Convergence analysis of some algorithms for solving nonsmooth equations. Mathematics of Operations Research, 18:227-244, 1993.

L. Qi and J. Sun. A nonsmooth version of Newton's method. Mathematical Programming, 58: 353-368, 1993.

F. Rathgeber, G. R. Markall, L. Mitchell, N. Loriant, D. A. Ham, C. Bertolli, and P. H. J. Kelly. Pyop2: A high-level framework for performance-portable simulations on unstructured meshes. In High Performance Computing, Networking Storage and Analysis, SC Companion:, pages 11161123, Los Alamitos, CA, USA, 2012. IEEE Computer Society. ISBN 978-1-4673-3049-7. doi: 10. 1109/SC.Companion.2012.134. URL http://dx.doi.org/10.1109/SC.Companion.2012.134.

F. Rathgeber, D. A. Ham, L. Mitchell, M. Lange, F. Luporini, A. T. T. McRae, G. T. Bercea, G. R. Markall, and P. H. J. Kelly. Firedrake: automating the finite element method by composing abstractions. ACM TOMS, 43:24:1-24:27, 2016.

P. A. Raviart and J. M. Thomas. A mixed finite element method for 2-nd order elliptic problems. In Mathematical aspects of finite element methods, pages 292-315. Springer, 1977.

B. Riviére and M. Wheeler. Discontinuous Galerkin methods for and transport in porous media. Communications in Numerical Methods in Engineering, 18:63-68, 2002.

J.F. Rodrigues. Obstacle Problems in Mathematical Physics, volume 134. Elsevier, 1987. 
M. E. Rognes, R. C. Kirby, and A. Logg. Efficient assembly of h(div) and h(curl) conforming finite elements. SIAM Journal on Scientific Computing, 31(6):4130-4151, 2009.

M. Sala, J. J. Hu, and R. S. Tuminaro. ML3.1 Smoothed Aggregation User's Guide. Technical Report SAND2004-4821, Sandia National Laboratories, 2004.

K. Shahbazi. Short note: An explicit expression for the penalty parameter of the interior penalty method. Journal of Computational Physics, 205(2):401-407, May 2005. ISSN 0021-9991. doi: 10.1016/j.jcp.2004.11.017. URL http://dx.doi.org/10.1016/j·jcp.2004.11.017.

Z. Sheng and G. Yuan. A new nonlinear finite volume scheme preserving positivity for diffusion equations. Journal of Computational Physics, 315:182-193, 2016.

A. Signorini. Sopra alcune questioni di statica dei sistemi continui. Annali della Scuola Normale Superiore di Pisa-Classe di Scienze, 2(2):231-251, 1933.

A. Signorini. Questioni di elasticità non linearizzata e semilinearizzata. Rendiconti Di Matematica e Delle Sue Applicazioni, V. Serie, 18:95-139, 1959. ISSN 0034-4427.

F. I. Stalkup. Miscible displacement. Society of Petroleum Engineers, 1983.

D. Z. Turner, K. B. Nakshatrala, M. J. Martinez, and P. K. Notz. Modeling subsurface water resource systems involving heterogeneous porous media using the variational multiscale formulation. Journal of Hydrology, 428-429:1-14, 2011.

M. Ulbrich. Semismooth Newton Methods for Variational Inequalities and Constrained Optimization Problems in Function Spaces. Society for Industrial and Applied Mathematics, Philadelphia, PA, USA, 2011.

D. A. von der Schulenburg, T. R. R. Pintelon, C. Picioreanu, M. C.M. Van Loosdrecht, and M. L. Johns. Three-dimensional simulations of biofilm growth in porous media. AIChE Journal, 55(2): 494-504, 2009.

C. E. Knutson C. J. Werth and A. J. Valocchi. Pore-scale simulation of biomass growth along the transverse mixing zone of a model two-dimensional porous medium. Water Resources Research, 41(7), 2005.

X. H. Zhao, K. H. Kwek, J. B. Li, and K. L. Huang. Chaotic and resonant streamlines in the ABC flow. SIAM Journal on Applied Mathematics, 53:71-77, 1993. 